\title{
Galactic and Extragalactic Sources of Very High Energy Gamma-rays
}

\author{
D. Bose ${ }^{1, a}$, V. R. Chitnis ${ }^{2}$, P. Majumdar ${ }^{3,4}$, and A. Shukla ${ }^{5}$
}

1 Department of Astrophysics and Cosmology, S N Bose National Centre for Basic Sciences, Kolkata, India

2 Department of High Energy Physics, Tata Institute of Fundamental Research, Mumbai, India

3 High Energy Nuclear and Particle Physics Division, Saha Institute of Nuclear Physics, HBNI, Kolkata, India

4 Faculty of Physics and Applied Informatics, Department of Astrophysics, University of Lodz, Poland

${ }^{5}$ Discipline of Astronomy, Astrophysics and Space Engineering, Indian Institute of Technology, Indore, India

\begin{abstract}
Very high energy $\gamma$-rays are one of the most important messengers of the non-thermal Universe. The major motivation of very high energy $\gamma$-ray astronomy is to find sources of high energy cosmic rays. Several astrophysical sources are known to accelerate cosmic rays to very high energies under extreme conditions. Very high energy $\gamma$-rays are produced at these astrophysical sites or near through interactions of cosmic rays in the surrounding medium close to the sources. Gamma-rays, being neutral, travel in a straight line and thus give us valuable information about the cosmic ray sources and their surroundings. Additionally, very high energy $\gamma$-ray astronomy can probe many fundamental physics questions. Ground-based $\gamma$-ray astronomy began its journey in 1989 when Whipple telescope detected $\mathrm{TeV} \gamma$-rays from the Crab, a pulsar wind nebula in the Milky Way. In the last two decades, technological improvements have facilitated the development of the latest generation of very high energy detectors and telescopes which have delivered exciting new results. Until now over two hundred very high energy $\gamma$-ray sources, both galactic and extra-galactic has been detected. These observations have provided a deeper insight into a large number of important questions in high energy astrophysics and astroparticle physics. This review article is an attempt to enumerate the most important results in the exciting and rapidly developing field of very high energy $\gamma$-ray astronomy.
\end{abstract}

\section{Introduction}

The origin of cosmic rays is one of the most fundamental problems in high energy astroparticle physics. Cosmic rays, which are charged particles, mainly protons and

\footnotetext{
a e-mail: debanjan.bose@bose.res.in
} 
other nuclei, isotropically arriving at Earth, form an important component of the nonthermal universe. Cosmic ray spectrum, measured by various experiments since their discovery in the early twentieth century, spans over almost 13-14 orders of magnitude starting around $10^{9} \mathrm{eV} 1$. However, it is difficult to identify the sources, as cosmic rays, being charged particles, are deflected by intergalactic magnetic fields and do not point to sources of their origin. It is however well known that whenever charged particles are accelerated to relativistic energies, $\gamma$-rays are produced through various processes. So study of $\gamma$-rays can give valuable insights into cosmic ray acceleration. Also, $\gamma$-rays, being neutral are not deflected and point back to their progenitors, and thereby enable us to identify sources, where cosmic rays are produced and accelerated. Thus very high energy (VHE) $\gamma$-rays produced via non-thermal processes enable us to probe the most violent astrophysical events in our Universe.

Apart from the possibility of solving the century-long puzzle of cosmic ray origin, a motivation for the study of VHE $\gamma$-rays arises from the fact that they also give important clues about the emission regions and emission mechanisms of various astrophysical sources. VHE $\gamma$-rays can also help us to probe frontiers in physics, e.g. it can help us to study the nature of dark matter through indirect detection, understand photon propagation under quantum gravitational effects etc.

Over the last two decades, the field of VHE $\gamma-$ ray astronomy has been rapidly evolving with more than two hundred discoveries and detections of very high energy gamma-ray sources of different genres and classes. An exhaustive review of all the sources and its classes is beyond the scope of this article. Here we try to highlight some of the most important detections and their implications. This article is organised as follows : Mechanisms for charged particle acceleration and consequent VHE $\gamma$-ray emission are explained in section 2, Brief overview of sources detected by ground-based telescopes is presented in section 3 . Results from observations of various galactic and extragalactic sources are discussed in sections 4- 6. Finally, section 7 deals with the fundamental physics aspects which are addressed using VHE $\gamma$-ray observations followed by conclusions in section 8 .

\section{Emission Mechanisms for VHE $\gamma$-rays}

As mentioned earlier, lower energy emission from astrophysical sources is quite often thermal radiation. For example, optical emissions from stars are of thermal origin and are characterised by black body radiation with energy emitted being proportional to the temperature of the source. On the other hand, very high energy emissions arise from astrophysical objects powered by the release of gravitational energy and the relativistic acceleration of particles. The emission mechanisms for VHE $\gamma$-rays involve charged particles, leptons or hadrons which are accelerated to high energies. Possible mechanisms for particle acceleration include,

A. Second order Fermi or stochastic acceleration : It was proposed by Enrico Fermi in 1949 2] that charged particles colliding with clouds, associated with irregularities in the Galactic magnetic field, in the interstellar medium (ISM), could be accelerated to high energies. Particles gain energy stochastically in these processes. In the rest frame of the cloud, particles will be scattered elastically, however, if the clouds are moving then particles gain energy. Under relativistic conditions, change in particle energy can be written as $\frac{\Delta E}{E} \propto\left(\frac{V}{c}\right)^{2}$, where $\mathrm{V}$ is the speed of the cloud. Since the gain in energy is proportional to $\left(\frac{V}{c}\right)^{2}$, it is called Fermi second order acceleration. The energy gain process in this case is very slow since the speed of the cloud is much less than particle speed.

B. First order Fermi or diffusive shock acceleration : It was also proposed by Fermi [3.4] that a shock wave in plasma can accelerate particles efficiently. In this scenario, 
as the shock wave moves, charged particles cross the shock front multiple times back and forth as the magnetic field associated with the shock scatters them. This results in the acceleration of particles to relativistic energies. Every time a particle crosses the shock front, from either side, it gains energy. If $V_{s}$ is the velocity of the shock front then the energy gained by the particle is given by, $\frac{\Delta E}{E} \propto \frac{V_{s}}{c}$. As energy gained is proportional to $\frac{V_{s}}{c}$, this mechanism is also known as first order Fermi acceleration mechanism.

C. Magnetic reconnection : This is due to the breaking and rejoining of magnetic field lines in a highly conducting plasma. This process converts magnetic energy into plasma kinetic energy and thermal energy and accelerates particles.

Various possible mechanisms for VHE $\gamma$-ray emission, with leptonic as well as hadronic origin, are summarised below.

\subsection{Leptonic origin of $\gamma$-rays}

The energetic electrons can produce $\gamma$-rays via inverse Compton scattering, synchrotron emission, bremsstrahlung and curvature radiation [5]. Electrons are accelerated inside astrophysical sources usually have power-law distribution of the form, $\phi_{e} \propto E_{e}^{-p}$, where $p$ is the power-law index and $E_{e}$ is the energy of the electron.

\subsubsection{Inverse Compton Scattering}

In inverse Compton (IC) scattering, low energy photons, such as cosmic microwave background (CMB), infrared, optical or X-ray photons get up-scattered by relativistic electrons. Electrons transfer part of their energy to photons.

$$
e^{-}+\gamma_{\text {low }} \rightarrow e^{-}+\gamma_{\text {high }}
$$

If the energy of the photon $(\epsilon)$, before scattering is well below the rest mass energy of the electron $\left(\Gamma \epsilon<<m_{e} c^{2}\right.$, where $\Gamma$ is the Lorentz factor of the relativistic electron and $\Gamma \epsilon$ is the energy of the photon before scattering in the electron rest frame), then scattering takes place in Thomson regime.

Cross-section for IC scattering in Thomson regime is given by,

$$
\sigma_{T}=\frac{8}{3} \pi r_{0}^{2}
$$

where $r_{0}$ is the classical radius of electron. If $E_{\gamma}$ is the energy of the photons after scattering and $E_{e}\left(=\Gamma m_{e} c^{2}\right.$ in the lab frame) is the energy of the electron then, $E_{e} \propto E_{\gamma}^{1 / 2}$. Number of scattered photons in unit time can be estimated as,

$$
n \simeq \sigma_{T} c U_{r a d} / \epsilon
$$

where c is the speed of light and $U_{\text {rad }}$ is the density of the ambient photon field. Since $\sigma_{T}$ is independent of electron or photon energy, $\mathrm{n}$ is constant. Then energy spectrum of up-scattered photons is given by,

$$
\phi_{\gamma} \propto E_{\gamma}^{-(p+1) / 2}
$$

For energy $\Gamma \epsilon>>m_{e} c^{2}$, scattering happens in Klein-Nishina regime. Crosssection for scattering is,

$$
\sigma_{K N}=\frac{3}{8} \sigma_{T} x^{-1} \ln (4 x)
$$


where

$$
x=\frac{E_{e} \epsilon}{\left(m_{e} c^{2}\right)^{2}}
$$

Energy of the photon after scattering in the lab frame,

$$
E_{\gamma} \simeq \Gamma m_{e} c^{2}=E_{e}
$$

Number of scattered photons per unit time,

$$
n \simeq \sigma_{K N} c U_{r a d} / \epsilon \propto E_{e}^{-1}
$$

Assuming electrons have similar power-law distribution as in the previous case, energy spectrum of scattered photons will be,

$$
\phi_{\gamma} \propto E_{\gamma}^{-(p+1)}
$$

\subsubsection{Synchrotron radiation}

A charged particle emits electromagnetic radiation when it gyrates in presence of a magnetic field. Its trajectory is bent perpendicular to the direction of the magnetic field by the Lorentz force. This radiation is called synchrotron radiation. In relativistic regime, emission is beamed in the direction of the motion. If the electrons have a power-law spectrum $\propto E^{-p}$, then the synchrotron radiation spectrum is given by,

$$
\phi_{\gamma} \propto E_{\gamma}^{-(p+1) / 2}
$$

Synchrotron power emitted by the charged particle is $\propto 1 / m^{4}$, where $\mathrm{m}$ is the mass of the particle. Therefore this process is more efficient in case of the electrons and positrons compared to protons.

\subsubsection{Curvature radiation}

If an electron moves in presence of a curved magnetic field then it follows a curved trajectory. In such cases, electron emits curvature radiation. If the electrons have a power-law spectrum $\propto E^{-p}$, then the curvature radiation spectrum is given by,

$$
\phi_{\gamma} \propto E_{\gamma}^{-(p+1) / 3}
$$

\subsubsection{Bremsstrahlung}

When charged particle passes close to an atomic nucleus, it experiences a strong nuclear force, as a result, particle is accelerated and it emits radiation. The intensity of the emitted radiation is inversely proportional to the mass of the particle, this process is therefore efficient for electrons or positrons. The emitted radiation is beamed in the forward direction. The spectrum of the emitted radiation is given by

$$
\phi_{\gamma} \propto E_{\gamma}^{-p}
$$




\subsection{Hadronic origin of $\gamma$-rays}

\subsubsection{Decay of neutral pions}

The neutral pions $\left(\pi^{0}\right)$ are produced when accelerated hadrons, e.g. protons, interact with other protons or photons. In these collisions charged $\left(\pi^{+} / \pi^{-}\right)$and neutral pions $\left(\pi^{0}\right)$ are produced in equal numbers. Therefore $1 / 3 \mathrm{rd}$ of the pions produced are neutral. Charged pions will produce muons and neutrinos via decay (lifetime $\sim 10^{-8} s$ ). Two $\gamma$-ray photons are produced from decay of $\pi^{0}$ (lifetime $\sim 10^{-16} s$ ). This implies that about $1 / 6$ th of the primary energy is carried by each $\gamma$-ray produced in $\pi^{0}$ decay.

$$
p+p(\gamma) \rightarrow \pi^{0} \rightarrow 2 \gamma
$$

If flux of pions is defined by a power-law spectrum of the form,

$$
\phi_{\pi_{0}} \propto E_{\pi_{0}}^{-p}
$$

where $p$ is the power law index, then the resulting $\gamma$-ray spectrum can be estimated as,

$$
\phi_{\gamma} \propto E_{\gamma}^{-p}
$$

because $E_{\gamma}=1 / 2 E_{\pi_{0}}$ and $N_{\gamma}=2 N_{\pi_{0}}$. The spectrum of secondary pions follows the spectrum of the parent protons. It is generally believed that protons (cosmic rays) are accelerated following first order Fermi acceleration mechanism. In that case, the expected power-law index for protons at the source is -2 . It means $\gamma$-rays produced via hadronic processes will also have $E^{-2}$ spectrum. Since charged and neutral pions are produced together in this process, detection of neutrinos would provide definitive evidence for cosmic ray sources.

\section{VHE $\gamma$-ray Sources}

The Crab nebula was the first source detected in VHE regime, with good statistical significance, by Whipple imaging atmospheric Cherenkov telescope (IACT) in the year 1989 6. After eleven years, by the year 2000, the total number of sources detected by ground-based telescopes, were only eight (see Fig. 11). Thus the initial progress was rather slow. The number of detected sources started increasing rapidly post 2005 and at present VHE $\gamma$-ray emission has been detected from more than 240 sources. This increase is due to an improvement in the sensitivity of IACTs. During the Whipple era, in order to detect Crab nebula at $5 \sigma$ significance level approximately 25 hours of observation time was required. Current generation stereoscopic arrays of IACTs, like H.E.S.S., MAGIC and VERITAS need just a few minutes of exposure to detect Crab nebula at $5 \sigma$ significance level. It is expected that, with the upcoming Cherenkov Telescope Array (CTA), with further an order of magnitude improvement in sensitivity compared to present generation telescopes, Crab nebula would be detected with $5 \sigma$ significance in less than a minute and the number of VHE $\gamma$-ray sources will be around thousand at the end of the current decade. Skymap of

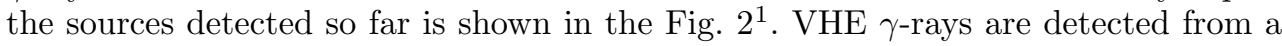
variety of cosmic sources. Amongst them, supernova remnants (SNR), pulsar wind nebula (PWN), pulsars etc. are located within our Milky Way Galaxy. VHE $\gamma$-rays are also observed from extragalactic sources come from relativistic jets of active galactic nuclei (AGN), from galaxies where stars are forming at an exceptional rate, known as starburst galaxies (SBG) and as afterglow emission from $\gamma$-ray bursts (GRB). There are many unidentified sources, the majority of which are located along the galactic plane, yet to be confirmed from observations in other wavebands.

\footnotetext{
1 http://tevcat.uchicago.edu/
} 


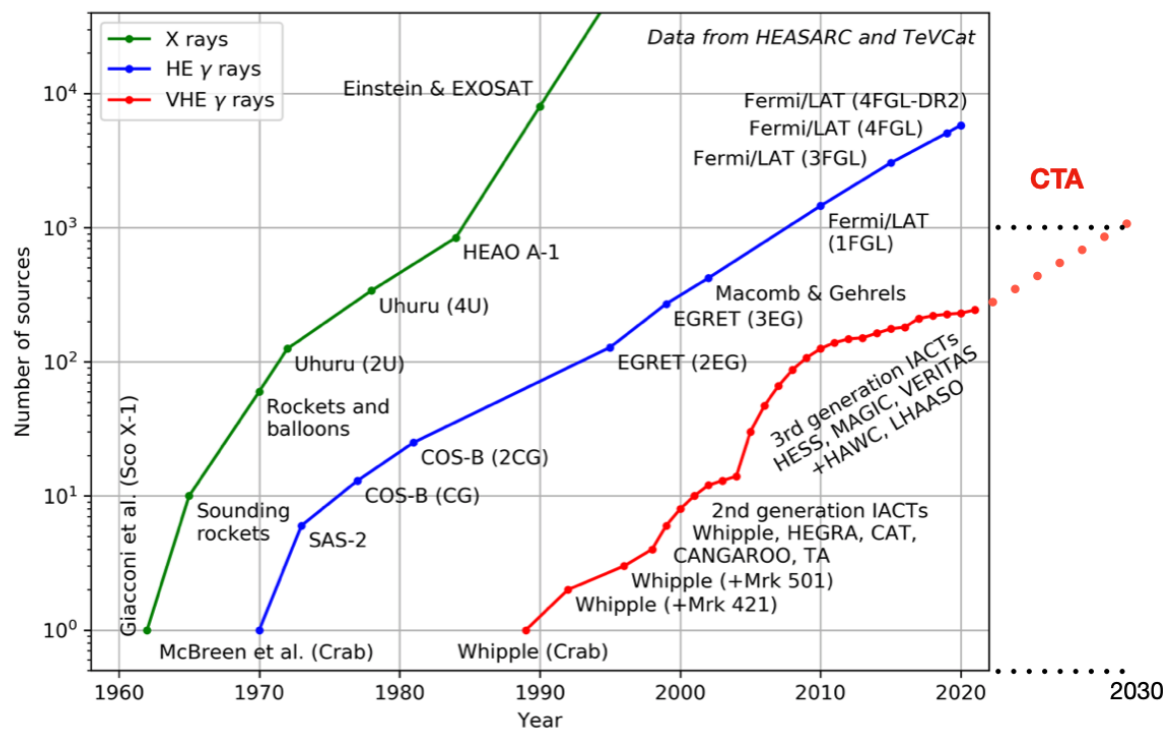

Fig. 1. Kifune plot. Number of sources detected in the last 80 years in X-ray, HE and VHE $\gamma$-rays. Adopted from (https://github.com/sfegan/kifune-plot) and modified to show expected number of sources detected by CTA in 2030 .

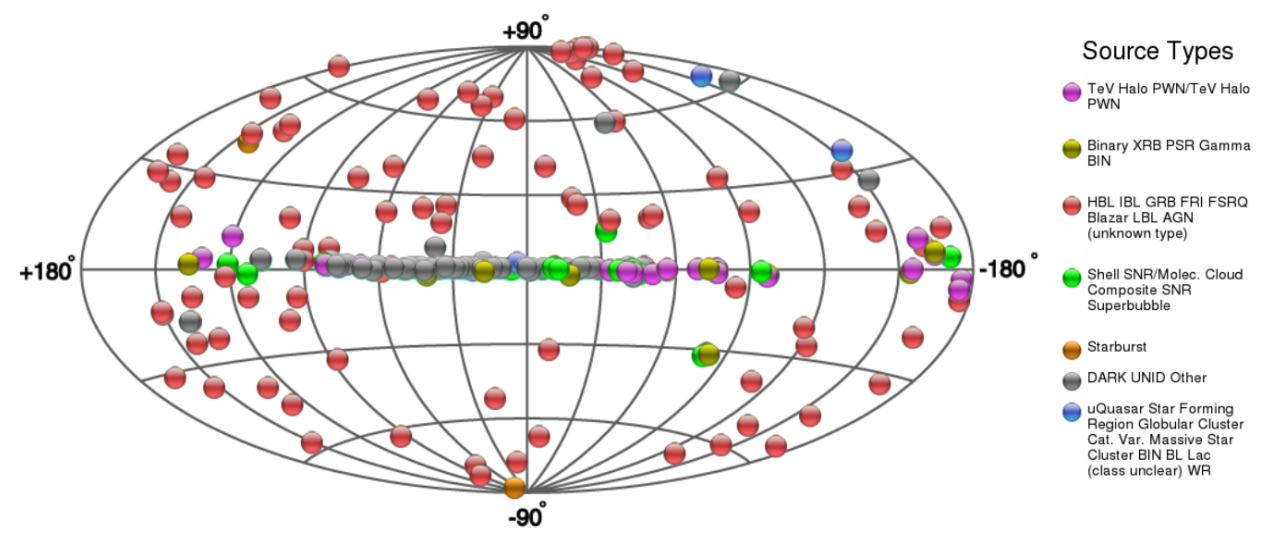

Fig. 2. Skymap of VHE $\gamma$-ray sources detected by ground based telescopes. Picture courtesy : TeVCat

\section{Galactic Survey}

As mentioned earlier, the current era of VHE $\gamma$-ray astronomy began with the detection of Crab nebula by the Whipple telescope [6]. Several galactic sources were observed after that. The most efficient way of detecting new sources in the galaxy is from the survey of the galactic plane. The first survey carried out by Whipple covering the range of $38.5^{\circ}<1<41.5^{\circ}$ and $-2^{\circ}<\mathrm{b}<2^{\circ}$, respectively, in galactic longitude and latitude produced a null result [7. This was followed by surveys conducted by HEGRA covering a wider range in galactic longitude and latitude 8, 9. The second survey, in fact, covered one-quarter of a galactic plane, but no evidence was found for VHE emission. Only with the present generation telescopes like H.E.S.S., VERITAS 
and MAGIC, survey of the galactic plane could be carried out in the VHE band with high resolution and good sensitivity. Located in the Southern hemisphere, H.E.S.S. is the most suitable instrument to study the galactic plane. The first survey covering the range of $\pm 30^{\circ}$ in longitude and $\pm 3^{\circ}$ in latitude around the galactic centre was reported in 2005 [10]. Ten sources were detected in this survey including eight previously unknown sources. Further extension of this survey increased the number of newly detected sources to 14 [1].

The most recent survey reported by H.E.S.S. was based on data collected over a decade during 2004-2013. It covered a wide range in galactic longitude $\left(250^{\circ}<1<\right.$ $\left.65^{\circ}\right)$ and latitude $\left(-3^{\circ}<\mathrm{b}<3^{\circ}\right)$ spanning 2700 hours of data 12 . This was a highly sensitive survey with the sensitivity of $\leq 1.5 \%$ of Crab flux for point sources and high angular resolution $\left(0.08^{\circ} \approx 5\right.$ arcmins mean point spread function $68 \%$ containment radius) over the energy range of $0.2-100 \mathrm{TeV}$. Total $78 \mathrm{VHE}$ sources were detected in this survey, out of which 31 were identified as pulsar wind nebulae (PWN), supernova remnants (SNRs), composite SNRs or $\gamma$-ray binaries (see Fig. 3). Remaining 47 sources were not identified clearly, having multiple possible counterparts from other wavebands or with no promising counterpart. Apart from this, VERITAS, located in the Northern hemisphere, also carried out survey of Cygnus region [15. The survey done over a period of 7 years accumulated more than 300 hours of data and detected a host of sources in the Cygnus region. The most notable work carried out in this survey has been in resolving the extended VHE $\gamma$-ray source VER J2019+368 into two source candidates (VER J2018+367* and VER J2020+368*) and the morphological studies on the SNR region Gamma-Cygni.

A survey of the Northern sky has also been carried out using the water Cherenkov observatory HAWC. Based on 1523 days of data, 65 sources have been detected at energies above several TeV [16]. Barring two blazars, a large fraction of these sources are from the galactic plane and many of them have pulsars as potential counterparts.

It is extremely interesting to compare the populations of high energy $\gamma$-ray sources detected by Fermi-LAT and VHE $\gamma$-ray sources detected by H.E.S.S. and VERITAS telescope arrays. In the region surveyed by H.E.S.S., 78 sources were detected as opposed to only 4 or 5 in the northern survey ${ }^{2}$. Whereas Fermi-LAT discovered 339 sources in the southern hemisphere within the survey region of H.E.S.S. and 37 in the northern hemisphere. One would have expected about 8 to 9 sources to be detected. This simplistic calculation suggests that there is likely a correlation between the numbers of HE and VHE sources in a region even though this estimate does not take into account the distances to the sources, the possibility of source confusion, the differences in the diffuse Galactic background and several other factors.

Various classes of galactic sources and some important results from VHE $\gamma$-ray observations of these sources are discussed in following subsections.

\section{Galactic Sources}

In the last two decades many galactic sources are detected in very high energy regime. These VHE $\gamma$-rays bring us important information about emission mechanisms and the nature of galactic sources. Another important motivation to study VHE $\gamma$-ray emission from these objects is to find sites for cosmic ray acceleration. The cosmic ray flux measured on earth is characterised by a power-law spectrum. This power-law spectrum has a break around $\mathrm{PeV}\left(10^{15} \mathrm{eV}\right)$ energy, known as "knee". It is believed that cosmic rays with energies below the "knee" could be accelerated in galactic

\footnotetext{
2 one should note that the sensitivity of the H.E.S.S. observations was a factor 2 better than that of VERITAS
} 


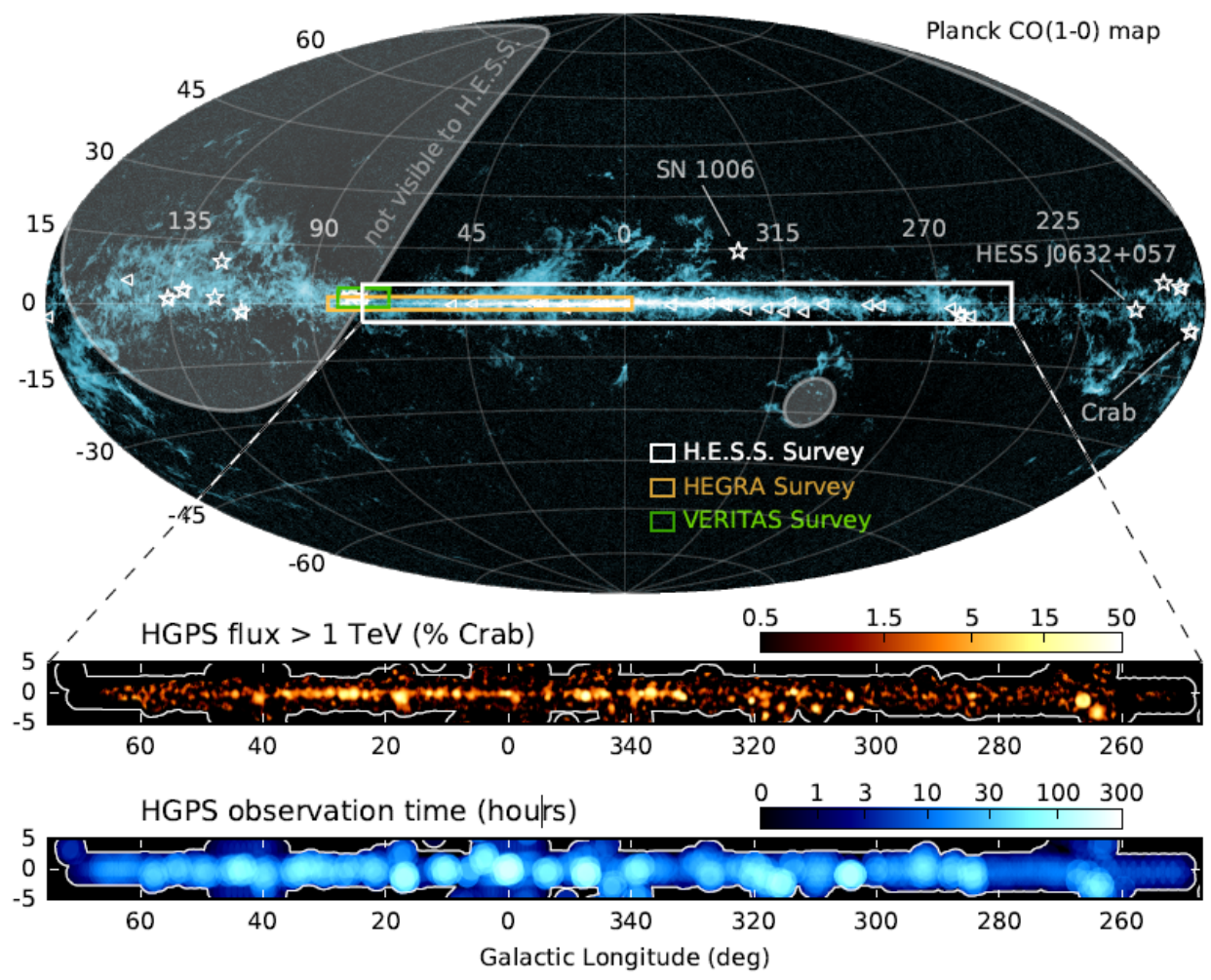

Fig. 3. H.E.S.S. galactic plane survey region superimposed on the all-sky image of Planck $\mathrm{CO}(1-0)$ data 13 in galactic coordinates. HEGRA Galactic plane survey 9 and VERITAS Cygnus survey [14] footprints are overlayed for comparison. Lower panels show $\gamma$-ray flux above $1 \mathrm{TeV}$ as detected by HESS and observation time. See [12] for further details from where the figure is reproduced.

sources. Supernova Remnants have long been thought to be likely candidates for accelerating cosmic rays. Also, there are other types of galactic sources, like pulsars, pulsar wind nebulae and X-ray binaries which are found to be VHE $\gamma$-ray emitters and could also be acceleration sites for cosmic rays. Many of these are remnants of supernova explosions of massive stars.

\subsection{Supernova Remnants}

Massive stars end their life through supernova explosions forming neutron stars or black holes. These explosions blow off the outer layers of the stars into ISM forming supernova remnants (SNRs). Cosmic rays are believed to be accelerated to multi-TeV energies in shock waves of supernova remnants via the mechanism of diffusive shock acceleration [17, 18, 19,20,21,22. It has been long estimated that if about $10 \%$ of the energy of the explosion is converted to cosmic rays, SNRs will be able to maintain the flux of galactic cosmic rays at the observation level. Several classes of SNRs are thought to be potential sites of cosmic ray acceleration : (a) composite SNRs are the ones with an energetic pulsar at its center, (b) shell type SNRs which are expected to be very efficiently accelerating particles to TeV energies. The older SNRs are thought to be shining mostly in $\mathrm{GeV}$ energies and the younger ones in $\mathrm{TeV}$ energies. Their 
evolution can be probed through multiwavelength observations [23. In the H.E.S.S Galactic Plance Survey, a few SNRs have been clearly detected and their morphologies have been resolved in VHE $\gamma$-rays, namely, RX J1713.7-3946[24], RX J0852.04622 (also known as Vela Junior) 25, SN 1006 26], HESS J1731-347 27] and RCW 86 28. In the northern hemisphere, VERITAS and MAGIC collaborations have also detected a few SNRs : CasA 29,30,31, Tycho 32, IC 443 33, 34] to name a few. Almost all the SNRs detected by H.E.S.S show a clear correlation between X-rays and $\mathrm{TeV} \gamma$-rays. The ones detected in the northern hemisphere have also been detected by Fermi-LAT with the spectra peaking at $\mathrm{GeV}$ energies and are thought to be consistent with the existence of a "pion bump" which supports hadronic acceleration [35, 36, 37].

The brightest galactic X-ray SNR RX J1713.7-3946 was discovered by the CANGAROO collaboration at VHE energies 38 . Later H.E.S.S performed detailed observations of the source and studied its morphology and it was the first young shell-type SNR to be resolved in VHE $\gamma$-rays 24. The VHE morphology was found to be well correlated with X-rays (see Fig. 4). Recent observations of the shell in VHE and Fermi-LAT along with X-ray observations suggest that the dominant emission mechanism is leptonic in nature although hadronic emission could not be totally ruled out. Later studies by 39] who for the first time accounted for the possible thermal $\mathrm{X}$-ray emission showed that the lack of observed thermal emission line is evidence against pion decay to be the main source of $\mathrm{TeV}$ emission. In models, where pion decay is the dominant mechanism producing the observed $\mathrm{TeV}$ emission require high circumstellar medium densities and a low electron to proton ratio. These are not consistent with the observations carried out by Suzaku [40. However, a purely leptonic model based on a single population of electrons cannot explain the H.E.S.S 41, 42, and Fermi-LAT data 43 owing to the fact that the magnetic field derived break in the electron spectrum is $>100 \mu \mathrm{G}$ which is in conflict with direct measurements. More recent data from H.E.S.S allowed for the reconstruction of spatially resolved spectra with high resolution $\left(<0.05^{\circ}\right)$. This permitted a detailed comparison of morphology in both X-rays and VHE $\gamma$-rays. From these images, radial profiles can be determined which clearly shows the diffusion of particles outside the shell and possible escape of particles which can be of hadronic origin 44.

Another class of well studied SNR, namely belonging to the core-collapse SNR type and of similar age but slightly older than RX J1713.7-3946, is $\gamma$-Cygni, located in the heart of the Cygnus region. This source has been well studied with both VERITAS and MAGIC telescope systems [45, 46] in the VHE regime and Fermi-LAT in high energy $\gamma$-rays 47, 48. It hosts a pulsar PSR 2021+4026 discovered in a blind search by FermiLAT 49, which is also likely associated with the SNR. There are several components associated with this object : an interior portion, an extended emission outside the SNR and also an extended source named MAGIC J2019+408 in the north-west of the remnant. Detailed investigations and modelling of the region revealed that cosmic rays are escaping the shock of the SNR upstream into the ISM while less energetic cosmic rays are confined within the SNR shock.

IC443, also known as Jellyfish nebula, is a nearby extended SNR exhibiting a shell like morphology in radio and optical. It has a complex morphology and is thought to interact with molecular clouds (MC) reaching a very high density. The source was discovered in VHE $\gamma$-rays by both MAGIC 33. and VERITAS 34] and later by AGILE and Fermi-LAT [50] in the GeV domain. After the initial discovery, it was found out that the centroid of the $\mathrm{TeV}$ emission is coincident with a $\mathrm{MC}$ where an $\mathrm{OH}$ maser emission is also found (see Fig. 5). Later, using more than 5 years of observations with Fermi-LAT, the characteristic pion-decay feature in the $\gamma$-ray spectra of IC 443 was established which for the first time provided evidence that protons are accelerated in SNRs 36]. 


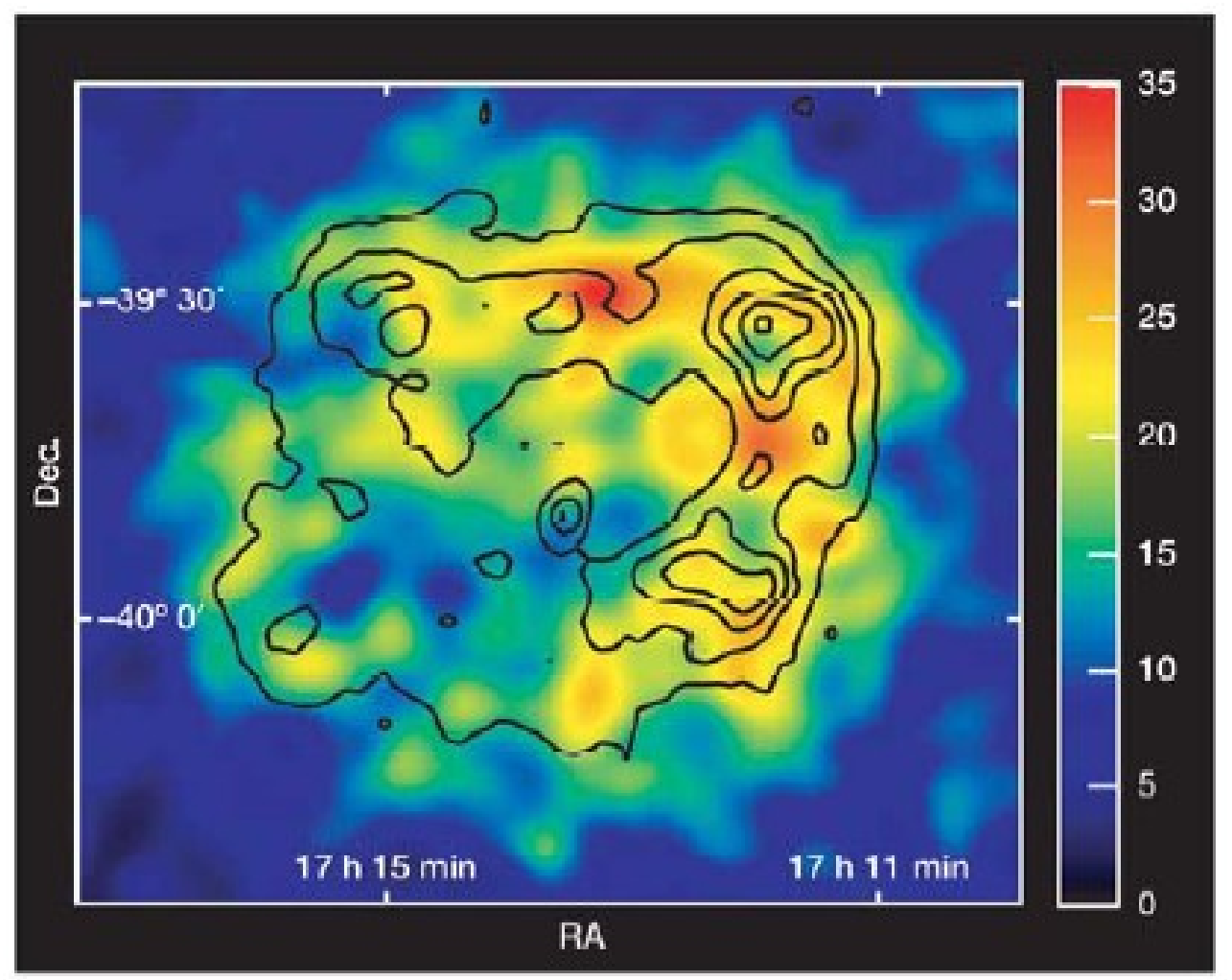

Fig. 4. VHE $\gamma$-ray image of RX J1713-3946. The X-ray surface brightness as measured by ASCA in 1-3 keV energy range is overlaid on the image. The figure is taken from 24

The supernova remnant RCW 86/ SN 185 is the oldest SNR. This source has been studied extensively across the electromagnetic spectrum. H.E.S.S collaboration first reported VHE emission from this source in 2009 28. Further deeper observations enabled a detailed morphological study of the source as shown in the Fig. 6 52 . This study confirmed that the source does have a shell like structure, which is also detected in other wavelengths. In X-ray energies this object shows some interesting characteristics, for example, it shows a softer spectrum at energies $<1 \mathrm{keV}$ and a harder spectrum at energies $>2 \mathrm{keV}$. This points to the fact that the hard X-ray emission is non-thermal in nature, i.e. it is produced due to synchrotron emission by electrons. The TeV $\gamma$-rays collected by H.E.S.S seems to correlate with hard X-rays, indicating $\mathrm{TeV}$ emission to be originating from upscattering of low energy photons to VHE regime, by the same population of electrons. This is called synchrotron selfCompton (SSC) scattering. However, the VHE data can also be explained by hadronic models, with protons colliding with the gas in the shell and producing neutral pions. But this would require a higher magnetic field and harder proton spectrum $\left(E^{-1.7}\right)$, not usual $\left(E^{-2}\right)$.

SNRs surrounded by molecular clouds are ideal candidates to be the sources of galactic cosmic rays. It is believed that protons or heavy nuclei accelerated by shocks, interact with molecular gas and produce neutral pions, which then decay to $\gamma$-rays. SNR W49B is one such source. HESS and Fermi-LAT collaboration jointly studied this source 53 . VHE emission can also have a leptonic origin, as discussed earlier. However, if VHE emission is due to leptonic interactions, then $\mathrm{HE} \gamma$-rays will have 

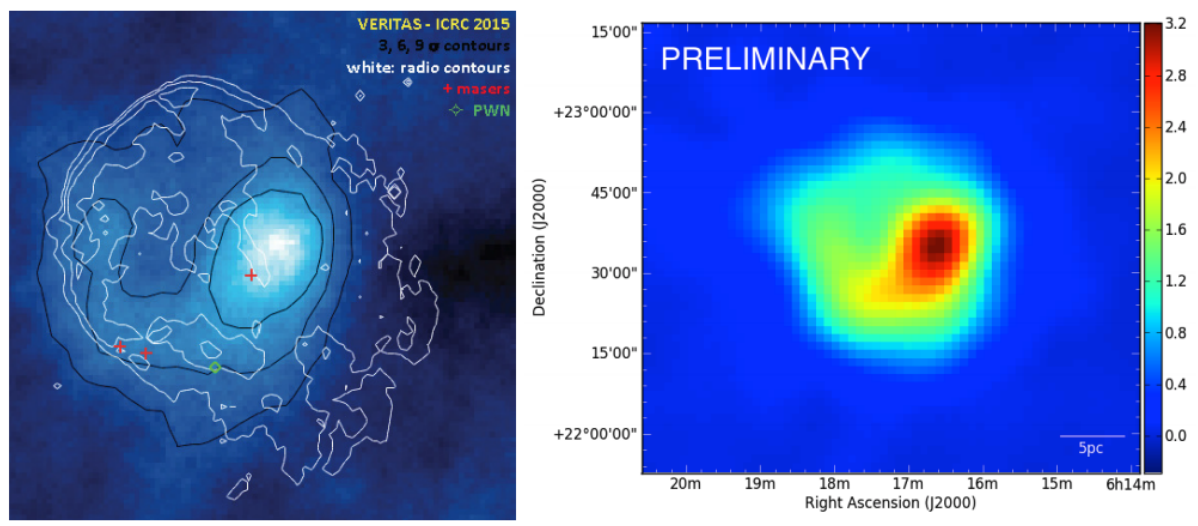

Fig. 5. Left: Excess map of IC443. The white contours indicate the presence of radio shell and the black contours designate VERITAS significance at 3, 6 and $9 \sigma$ levels. The red points show the locations of maser emission while the green one is a likely pulsar wind nebula. Right : The counts map obtained by Fermi-LAT with VERITAS significance contours overlaid on the map. The figure is taken from [51]

a steep rise below $200 \mathrm{MeV}$ (accessible by Fermi-LAT) as shown in the Fig. 7 . Even though the data obtained by HESS and Fermi-LAT are fitted with both hadronic and leptonic models, a sharp break around $300 \mathrm{MeV}$ gives an edge to the hadronic model.

Another source, RX J0852.0 4622 (also known as Vela junior), belonging to the class of young shell-type supernova remnants, has been recently extensively studied by H.E.S.S between 2004 and 2009 [54. Like RX J1713.7-3946, this source is also studied across the entire electromagnetic spectrum. Both these sources have similar characteristics. The $\gamma$-ray emission, as detected by Fermi-LAT and H.E.S.S is fitted well with both hadronic and leptonic models. In the leptonic scenario, $\gamma$-ray emission from RX J0852.0-4622 is dominated by the IC scattering of ambient radiation fields by relativistic electrons. The magnetic field strength of the northwestern rim estimated from X-ray data is a few $\mu \mathrm{G}$. The low magnetic field strength is in very good agreement with the parameters of different leptonic models. Leptonic models can also explain the cutoff energy observed around $20 \mathrm{TeV}$. However leptonic models are in contrast with the high magnetic field resolved by Chandra in the northwestern rim of the remnant. Such a high magnetic field favours the hadronic scenario. In the hadronic scenario, VHE emission requires a density of the ambient matter around $\sim 1 \mathrm{~cm}^{-3}$, whereas lack of thermal X-ray emission, indicates low density of ambient matter $\sim 0.01 \mathrm{~cm}^{-3}$. Protons can still produce the observed $\gamma$-rays with such low ambient density if they have energy $\sim 10^{51} \mathrm{ergs}$. An SNR generally releases $10^{51} \mathrm{ergs}$ and it is expected $10 \%$ of it is transferred to protons. Therefore if protons have energy $10^{51} \mathrm{ergs}$, which implies that all the energy of supernova explosion is transferred to protons. This problem can be solved if the SNR is expanding in an inhomogeneous environment. In that case, shock does not penetrate deep inside the clouds to produce thermal X-rays, but protons can and they can produce $\gamma$-rays by interacting with the matter in the cloud.

HESS J1826-130 is one of the brightest VHE sources discovered by H.E.S.S. in the galactic plane survey [12. VHE $\gamma$-rays above $10 \mathrm{TeV}$ are detected from this source [55]. There is evidence that this source is surrounded by dense molecular cloud, which means VHE $\gamma$-rays are likely to be produced by protons. Hard spectrum observed by H.E.S.S. can be explained under the hadronic scenario. Protons accelerated by nearby SNRs can interact with the gas and produce pions. Neutral pions then produce VHE $\gamma$-rays via decay. The hardness of the spectrum can also be explained under 


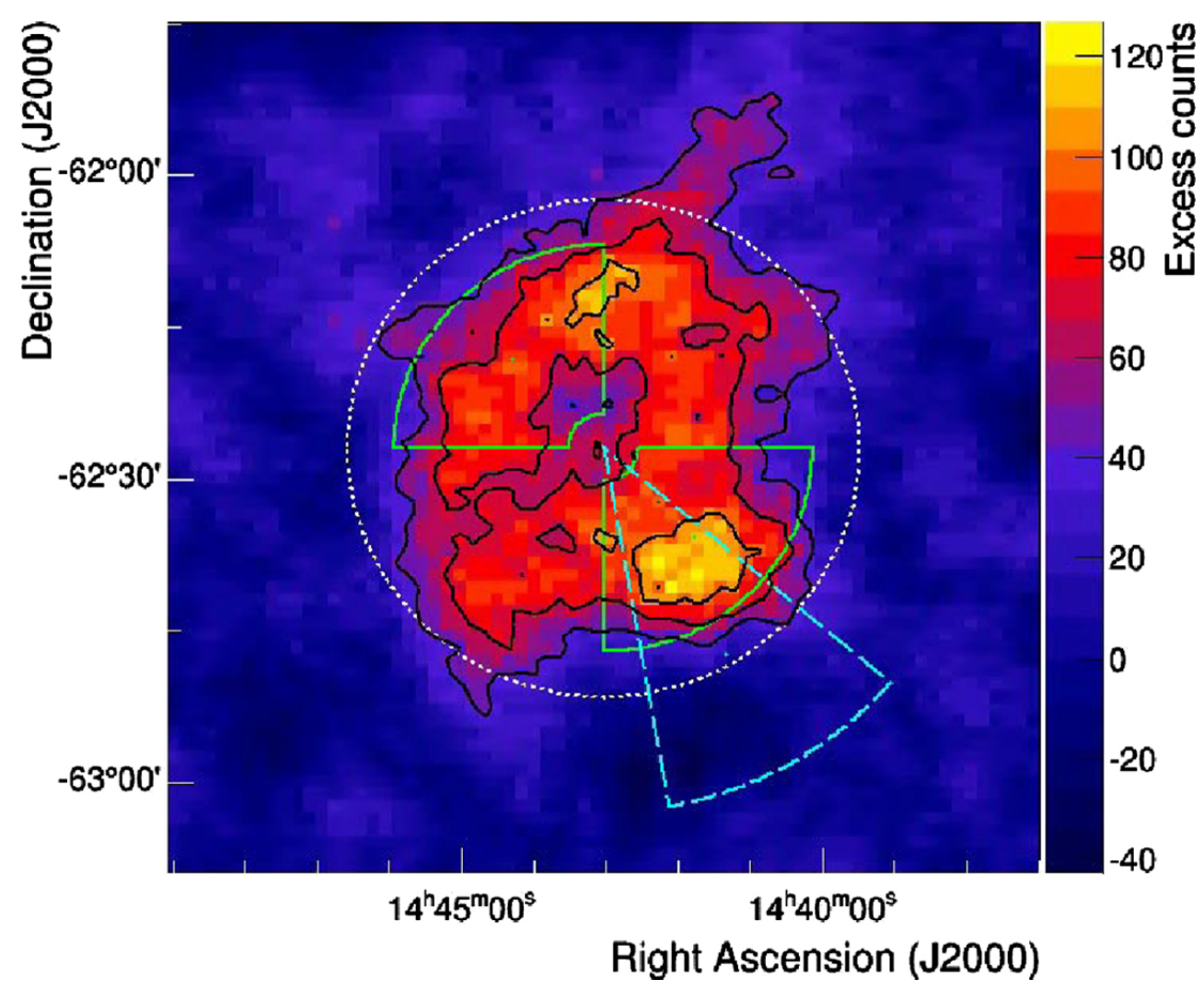

Fig. 6. Shell structure RCW 86 showing the VHE $\gamma$-ray emission region. The black contours correspond to 3,5 and $7 \sigma$ significance. The integration region of the analysis is shown by the white circle. The figure is taken from 52

leptonic scenario, e.g. electrons accelerated by pulsar can up-scatter cosmic microwave background (CMB) and IR photons to TeV energies. Recently, HAWC has reported detection of $\gamma$-rays above $100 \mathrm{TeV}$ from eHWC J1825-134 56] which encompasses the HESS J1826-130, indicating the fraction of the emission, at least upto around 40 $\mathrm{TeV}$, detected by HAWC is coming from this source.

\subsection{Pulsar Wind Nebulae}

Supernova explosion of a massive star sometimes results in rapidly rotating neutron star which is called a pulsar. Pulsars dissipate their rotational energies by throwing out relativistic winds of electrons and positrons in the ISM, which form a termination shock. In the case of an isolated pulsar, the rotational energy of the pulsar is converted into electromagnetic particle acceleration and the pulsar spin down provides a powerful energy source for the emission from these systems. In the pulsar magnetosphere, beyond the shock, a relativistic magnetised plasma is formed - known as pulsar wind nebula (PWN) (for a detailed review on the subject, please see [57 and references therein). The wind is an ultra-relativistic cold plasma of electrons, positrons and possibly ions (see Fig. 8). As PWNs can accelerate electrons and positrons very efficiently, they are believed to be the source of galactic leptonic cosmic rays. The broad-band emission from these objects is determined by the power and the spectrum of particles 


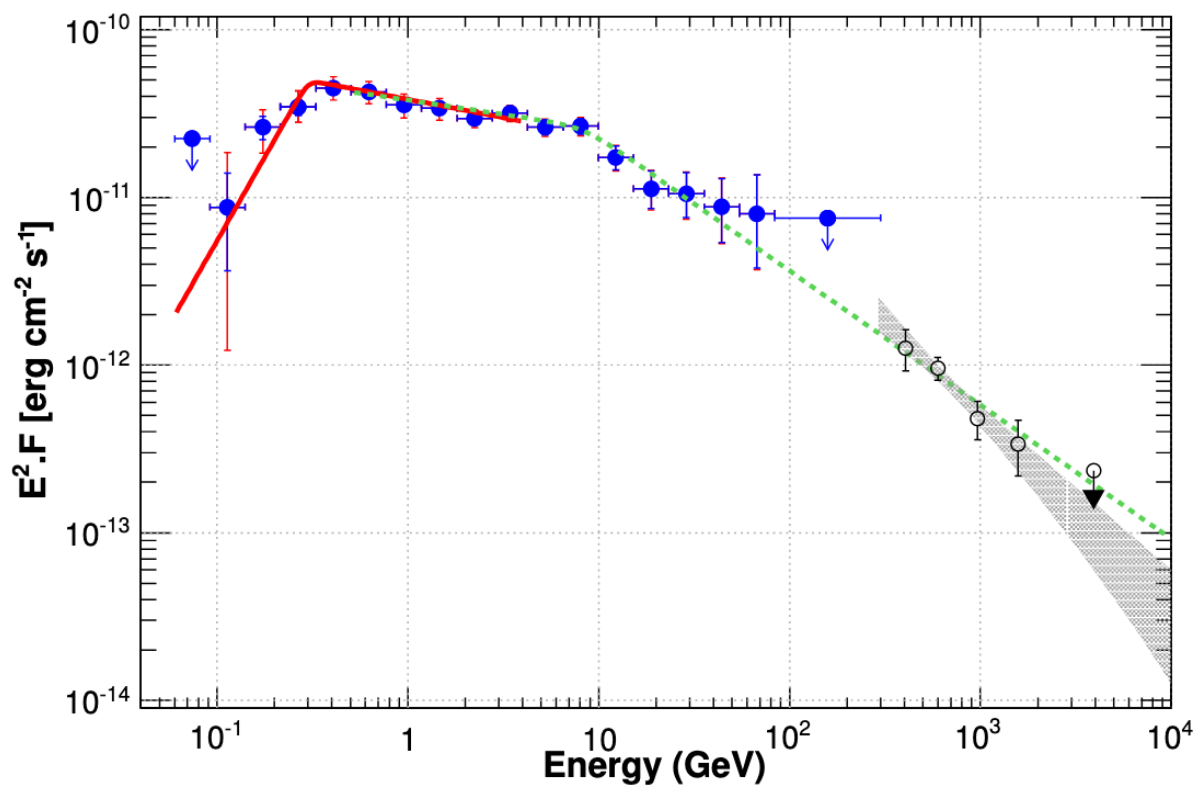

Fig. 7. The spectrum of W49B obtained using Fermi-LAT \& H.E.S.S. data. The figure is taken from 53

injected by the pulsar and also the medium in which the pulsar expands. The electrons and positrons injected into the nebula can produce synchrotron emission in the presence of magnetic field of the nebula. The radiation emitted by the relativistic electrons peaks at optical to X-ray energies, and $\mathrm{TeV} \gamma$-rays are produced by IC scattering of low energy photons (CMB or IR) by electrons. Thus, one can determine the particle densities from high energy $\gamma$-ray observations and the magnetic field strength can be determined from X-ray data. Hence, in order to understand the nature of PWNs, it is important to study these sources in both X-rays and VHE $\gamma$-rays. The most famous example of such a system is the Crab nebula which has been extensively studied in all wavelengths from radio to $\mathrm{TeV} \gamma$-rays. One of the most striking features is the difference in the spatial extent of the nebula when viewed at different energies. The size of the nebula shrinks with increasing energy, giving information on the cooling processes since high-energy particles injected into the nebula at the wind shock undergo both synchrotron and adiabatic energy losses. In addition to the synchrotron emission, the Crab Nebula also presents line emission in optical and thermal radiation in the infrared/sub-millimetre wavelength ranges. Crab nebula has been extensively observed by VHE $\gamma$-ray telescopes, since its first detection in VHE band by Whipple. Figure 9 shows the spectral energy distribution (SED) of the Crab Nebula from high energy to very high energy $\gamma$-rays. The first part, shown by dashed blue line is the synchrotron component and the second hump is the IC component. Recently, several experiments (MAGIC61, $\mathrm{AS}_{\gamma} 62, \mathrm{HAWC} 63$ and LHAASO 64 ) have reported the presence of $100 \mathrm{TeV} \gamma$-rays and beyond from the Crab, making it one of the most fascinating objects to study over several decades of energy, indicating the presence of extremely high energy particles $\left(\sim 10^{14-15} \mathrm{eV}\right)$ in the Crab.

A large number of objects discovered by the H.E.S.S. collaboration, in their galactic plane survey, are probably pulsar/PWN systems. H.E.S.S. observations have shown that most of the PWN are located in the inner region of the Milky Way, in the Crux 


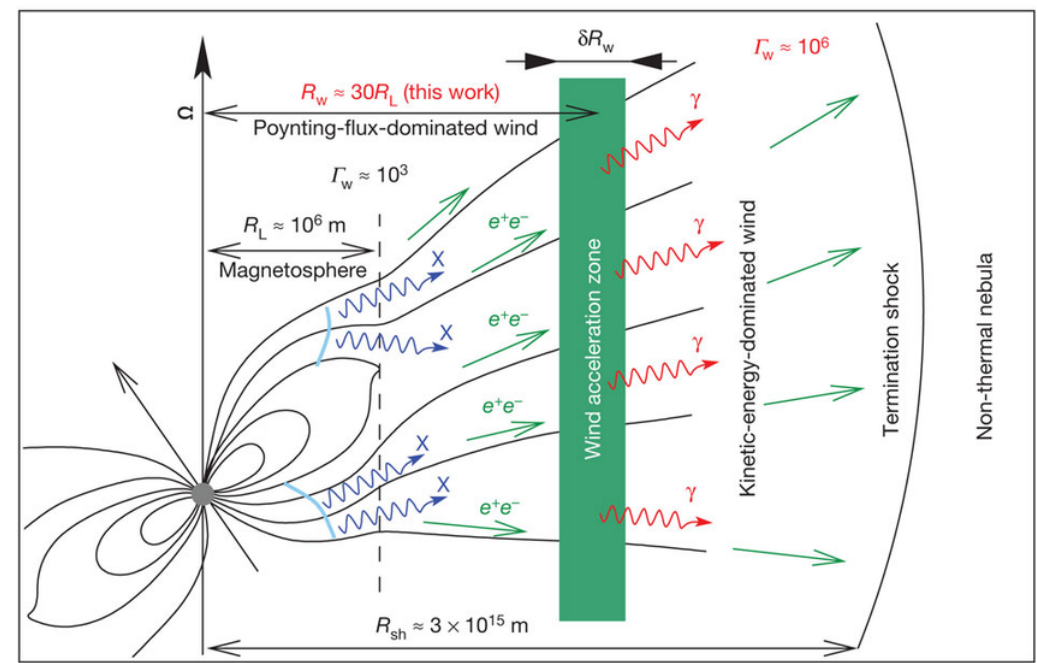

Fig. 8. Schematic of VHE $\gamma$-ray emission mechanism in a PWN. Reproduced from [58].

Scutum arm. Of the 17 most energetic pulsars in the Australia National Telescope Facility (ATNF) catalog, several of them (approximately 9) are associated with a $\mathrm{TeV}$ pulsar wind. A detailed correlation study confirms the picture that only young energetic pulsars are able to produce $\mathrm{TeV}$ pulsar winds which can be detected by the present generation of Cherenkov telescopes [65.

One such system on which deep $\gamma$-ray observations were conducted by H.E.S.S. is the PWN, HESS J1825-137 66], which hosts a pulsar PSRJ1826-1334 as the central powering engine and is one of the 20 most energetic pulsars in the ATNF catalog. The PWN is extended asymmetrically to the south and south-west of the pulsar. The energy dependent morphology (as shown in the Fig. 10) of this system is one of the first to have been studied in detail with photons beyond $30 \mathrm{TeV}$ seen in the analysis. The photons deteted at the VHE energy regime depict different power-law behaviours in different regions of the nebula and shows a softening of the spectrum with increasing distance from the pulsar. Very recently, LHAASO collaboration has reported the evidence of $>100 \mathrm{TeV}$ photons from this PWN at a very high statistical significance making this source to be a possible PeVatron 64.

H.E.S.S. collaboration studied Vela PWN and obtained SED for three compact regions 68. Using X-ray data from Suzaku for this study, the SED was fitted with a simple radiative model in which $\mathrm{X}$-ray photons are due to synchrotron emission by electrons and VHE $\gamma$-rays are due to IC scattering. The electron spectrum is found to have a cut-off at around $100 \mathrm{TeV}$ and the data is best fitted with a constant magnetic field. The derived electron spectra are consistent with expectations from cosmic ray transport scenarios dominated either by advection via the reverse shock or by diffusion. Future observations at high energy X-rays can give constraints on the magnetic fields and the shape of the electron cutoff.

In the northern hemisphere, the bright extended unidentified Milagro source, MGRO J2019+37, located towards the star formation region Cygnus-X [69, has been resolved into two sources by VERITAS 70]. The faint point-like source VER J2016+371 71 is coincident with CTB 87, a filled-center remnant (SNR) but shows no evidence of an SNR shell and is likely to be associated with an extended X-ray PWN. The bright extended object VER J2019+368 which accounts for the bulk of the emission detected by Milagro is coincident with the pulsar PSR J2021+3651 and the star formation region Sh 2-104 (see Fig. 11). The high spin-down luminosity of PSR 


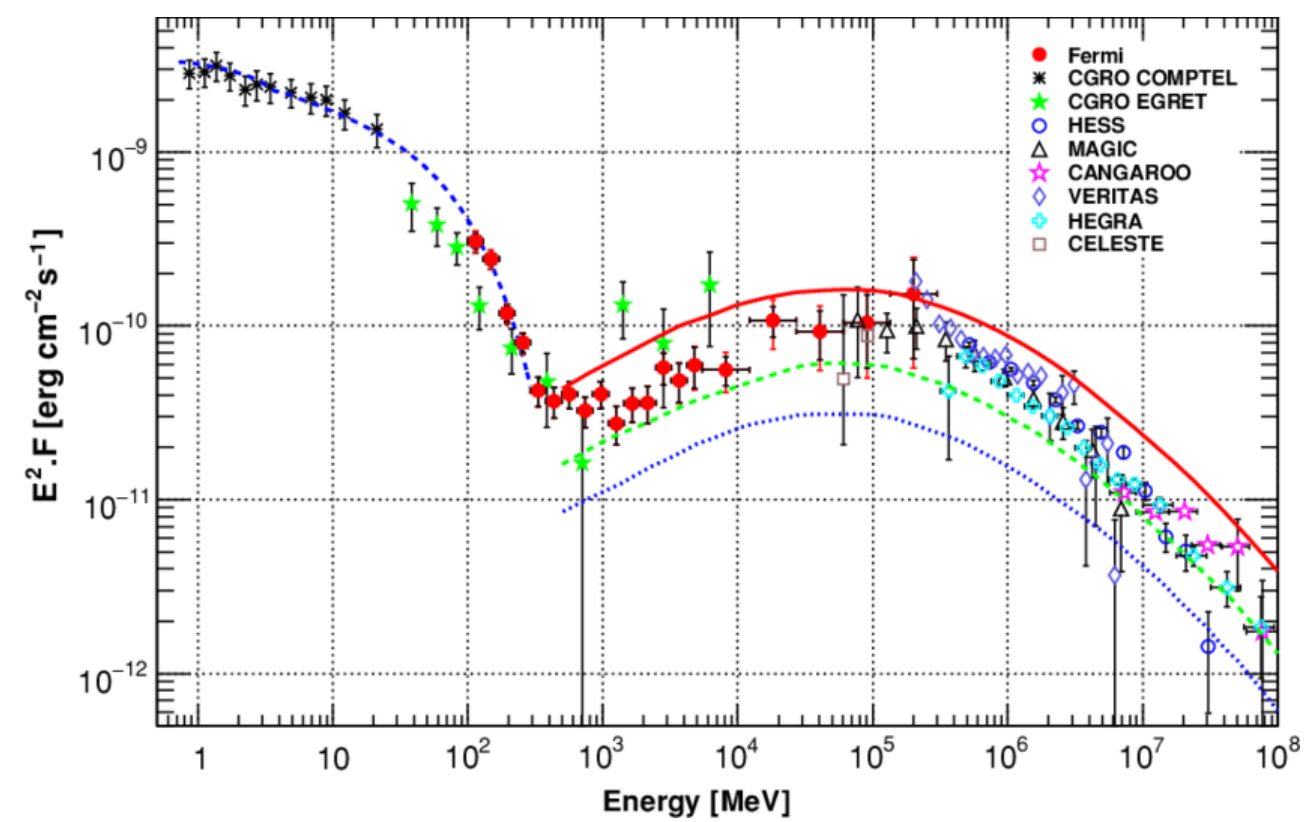

Fig. 9. The spectrum of Crab nebula from HE to VHE $\gamma$-rays. The data from CGROCOMPTEL and Fermi-LAT are fitted with synchrotron component. The spectral data with energy of $10^{3} \mathrm{MeV}$ and above are fitted with inverse Compton component. Data have been taken from various past and present atmospheric Cherenkov telescopes. Along with the data, predicted inverse Compton spectra [59] are plotted, for different magnetic fields : $100 \mu \mathrm{G}$ (solid red line), $200 \mu \mathrm{G}$ (dashed green line) and $300 \mu \mathrm{G}$ (dotted blue line) The figure is adapted from 60.

J2021+3651 makes plausible a PWN scenario for the emission of VER J2019+368. However, one cannot rule out the contribution from the star-forming region Sh 2-104 because of its proximity to the VHE emission region, although the mass of Sh 2-104 swept up seems to be low compared to other star-forming regions that are associated with VHE $\gamma$-ray sources, such as W49A 72 or Westerlund173.

\subsection{X-ray Binaries}

X-ray binaries consist of a compact object, either a neutron star or a black hole accreting matter from a companion star. Transfer of matter could be due to Roche lobe overflow forming an accretion disk around the compact object, in case of low mass companion, and through the stellar wind in case high mass companion like OB star. In some cases, companion is Be star with non-isotropic stellar wind forming an equatorial disk around the star. In some of the cases, relativistic outflows or jets have been noticed from compact objects, these are called microquasars. A small fraction of $\mathrm{X}$-ray binaries are found to emit VHE $\gamma$-rays. Eleven such objects have been detected at VHE $\gamma$-ray energies so far. Mechanisms for $\gamma$-ray emission in these binaries are summarised in 74 as shown in Fig. 12. In the case of a pulsar i.e. neutron star, there are strong pulsar winds powered by the rapid rotation of neutron star. If a companion is a massive star with high-density UV photon field, these photons can interact with relativistic particles in pulsar wind and reach $\gamma$-ray energies by IC scattering. If the companion is a Be star, pulsar wind particles interact with ions in the Be star disk and produce $\gamma$-rays. In the third scenario, corresponding to microquasars with a massive 

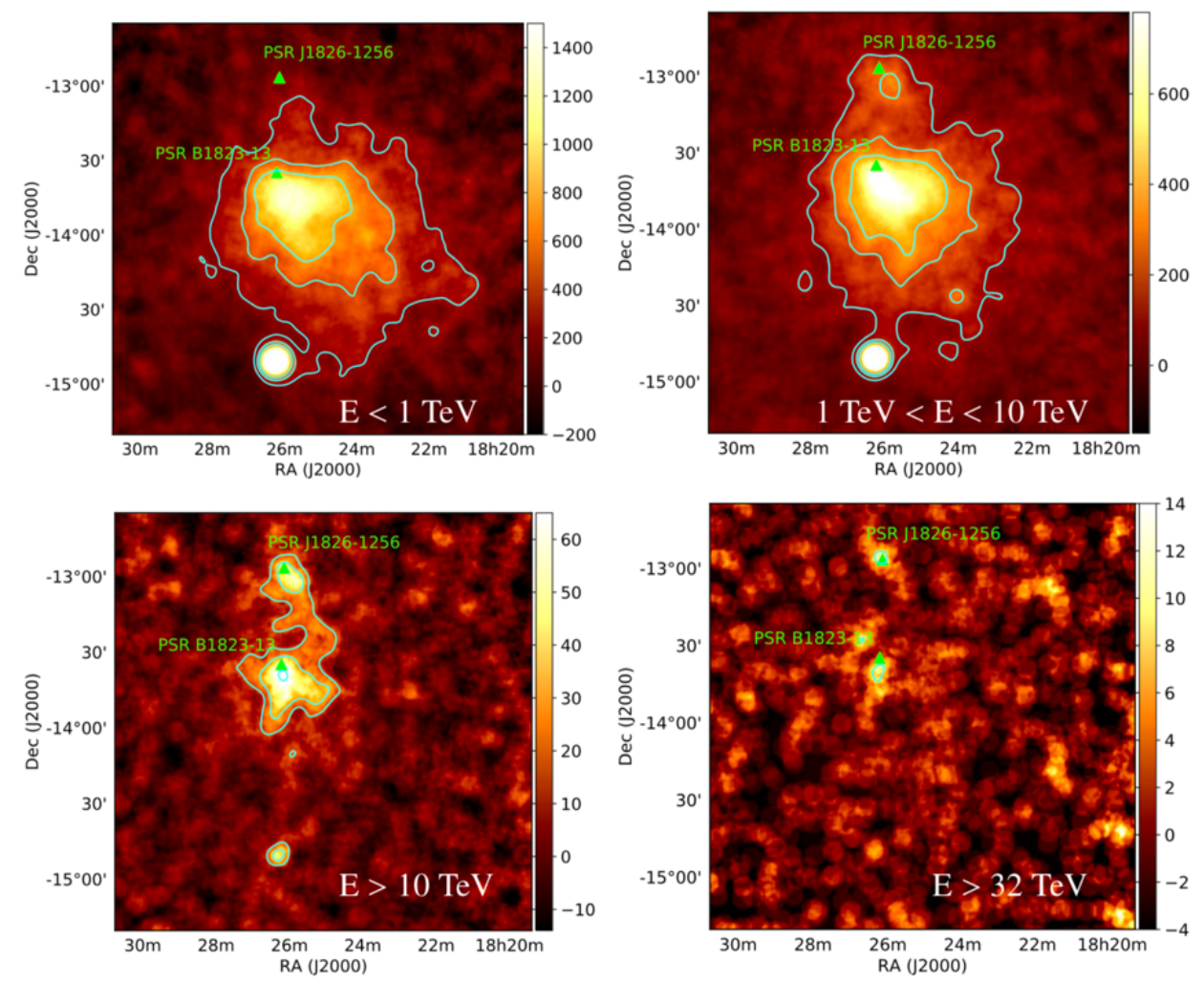

Fig. 10. Map of HESS J1825-137 showing the excess counts in different energy bands. There is a clear energy dependent morphology seen and the size of the nebula reduces as one goes to higher energies. The figure is taken from [67. The fact that the size of the nebula reduces with increasing energy is quite consistent with expectations of particle transport and cooling timescales over the lifetime of the nebula

star with a strong UV field as a companion, $\gamma$-rays can be produced by electron-proton or electron-photon interactions 74 .

The first microquasar discovered to be a VHE $\gamma$-ray emitter is LS 5039. VHE emission from this source was first detected by H.E.S.S. during the galactic plane survey which was confirmed by the follow-up observations 75. Earlier, radio observations of this source with VLBA discovered radio jets on the milli-arcsecond scale, indicating the object to be a microquasar 76 . This microquasar consists of a massive star of type $06.5 \mathrm{~V}$ with the compact object of an unknown type moving around it in an eccentric orbit. Further observations at VHE energies during 2004-05 showed modulation of VHE $\gamma$-ray flux with a period of 3.9 days [77, matching with the orbital period of the binary system estimated from optical observations [78. Most of the VHE $\gamma$-ray flux was found to confine to half of the orbit, peaking around inferior conjunction of the compact object, when both the objects in binary are aligned with our line of sight, with the compact object in front of the companion star. This modulation indicates $\gamma$-ray absorption in the source with a large part of the VHE $\gamma$-ray production region situated within $\sim 1 \mathrm{AU}$ of the compact object. Apart from flux modulation, hardening of the energy spectrum in the range between $0.2 \mathrm{TeV}$ and a few $\mathrm{TeV}$ is also seen near inferior conjunction. 


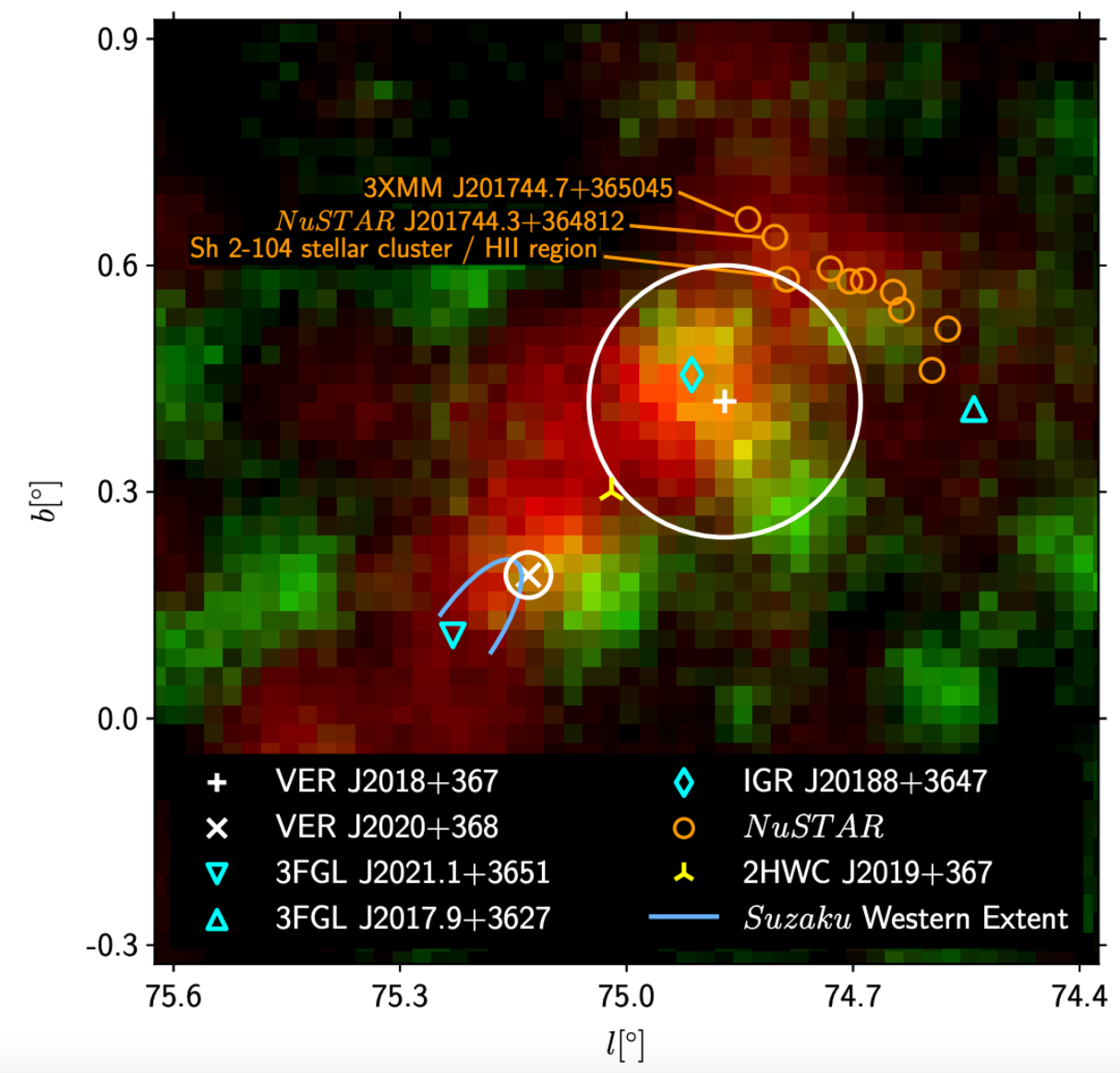

Fig. 11. Excess map for $>1 \mathrm{TeV}$ (red) and $<1 \mathrm{TeV}$ (green) for the region around VER J2019+368. The analysis has been performed for a point-like source. Two other sources, VER J2018+367 and VER J2020+368, are also shown (solid white lines and $1 \sigma$ extent). The sources show emission below $1 \mathrm{TeV}$. Locations of 2HWC J2019+367 (yellow, with the $1 \sigma$ error on its position), IGR J20188+3647 (cyan diamond), the two Fermi -LAT pulsars (3FGL J2021.1+3651 and 3FGL J2017.9+3627 (cyan triangles) are also shown in the map). The figure is taken from [15]

Soon after LS 5039, VHE $\gamma$-ray emission was detected from another micro-quasar, LS I $+61^{\circ} 303$ by MAGIC 79 . It consists of a Be star and a compact object of an unknown type orbiting around it with a period of 26.496 days [80,81. As in the case of LS 5039, this source also showed modulation of VHE $\gamma$-ray flux with a period close to the orbital period and stronger emission close to apastron (the point where separation between Be star and the compact object is maximum) and inferior conjunction rather than periastron (the point where separation between Be star and the compact object is minimum). Multiwaveband studies revealed a strong temporal correlation between X-ray and VHE $\gamma$-ray bands during observations carried out in September 2007 82, favoring the leptonic emission models over the hadronic ones. However, during observations carried out a little later, in 2008 and 2010 by VERITAS, the source showed quite different behaviour. There was no evidence of VHE $\gamma$-ray emission near apastron as seen earlier, instead, it was seen near superior conjunction, 

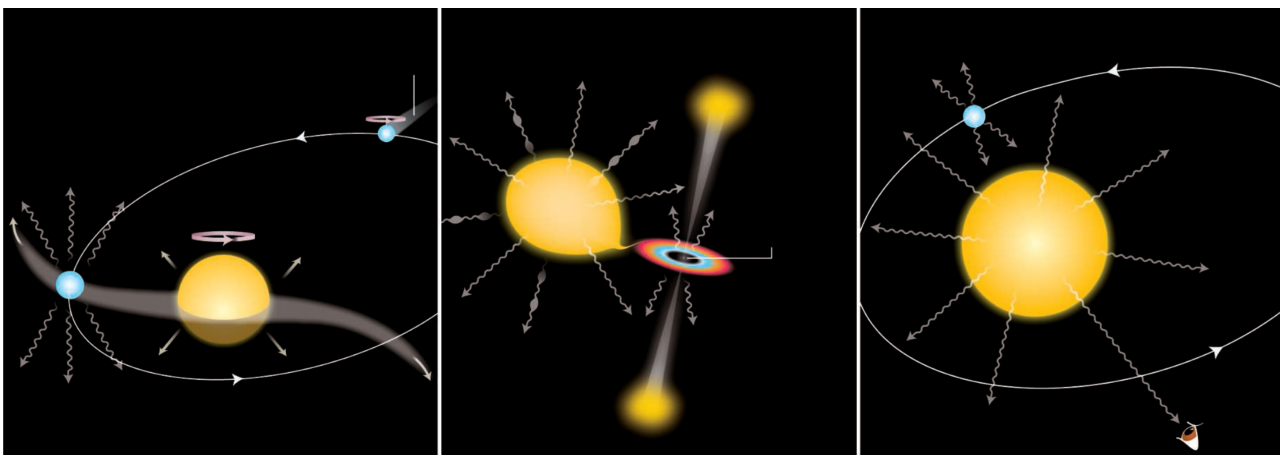

Fig. 12. $\gamma$-ray production a. by the interaction of pulsar wind particles with ions in Be star disk, b. in microquasars with a massive companion having strong UV field by electron-proton or electron-photon interactions, c. by interaction of pulsar wind with UV photons from the companion star. Adapted from 74] with permission.

closer to periastron [83. Also, no correlation was seen between X-ray and VHE $\gamma$-ray emission 84 indicating a major change in the source.

Another interesting binary system detected at VHE $\gamma$-ray energies during early observations by H.E.S.S. is PSR B1259-63. It consists of a pulsar with a spin period of $\sim 48 \mathrm{~ms}$ orbiting a massive B2e companion star in an eccentric orbit 85, 86. The closest approach or periastron with a distance of $\sim 10^{13}$ cms occurs every 3.4 years. VHE $\gamma$-ray emission from this object was detected by H.E.S.S. two weeks before the periastron and for a few months after that in 2004 [87. Strong VHE $\gamma$-ray emission was seen during pre- and post-periastron with a minimum around the periastron and emission gradually fading over subsequent months. Fig. 13 shows a sketch of the orbit of PSR B1259-63 and integral flux from various observations from H.E.S.S. during the observation period spanning the February-June 2004 period. Fig. 14 shows the VHE light curve from these observations along with the radio light curve. Subsequent observations carried out by H.E.S.S. around the next periastron passages in 2007 [89], 2010 [90, 2014 and 2017 91] showed similar flux variation.

VHE $\gamma$-ray emission was detected from few more binaries later by H.E.S.S. as well as VERITAS and MAGIC. Most of them are binaries consisting of a compact object of an unknown type and a Be star companion, with emission seen around the periastron. Apart from PSR B1259-63, there is one more binary PSR J2032+4127 consisting of a pulsar and a Be star 92. There are also other interesting objects like LMC P3, which is the first $\gamma$-ray binary detected outside Galaxy [93] and Eta Carina, which is a colliding wind binary system, consisting of two massive stars orbiting each other 94. Also, VHE $\gamma$-ray emission was detected from SS 433 by HAWC from the eastern and the western regions of relativistic jet originating from the black hole 95 .

\subsection{Pulsars}

As mentioned earlier, pulsars are highly magnetized rapidly rotating neutron stars formed in supernova explosions. As neutron star spins rapidly, charged particles are ripped away from the surface of the neutron star and accelerated along the magnetic field lines and produce electromagnetic radiation. Since the magnetic axis and rotation axis are not aligned, the beam of radiation originating from a specific region in magnetosphere sweeps through our line of sight and we see pulsations (see Fig. 15). Pulsed emission is seen from these objects in various wavebands from radio to $\gamma$-rays. The primary radiation mechanism is thought to be the synchrotron-curvature 


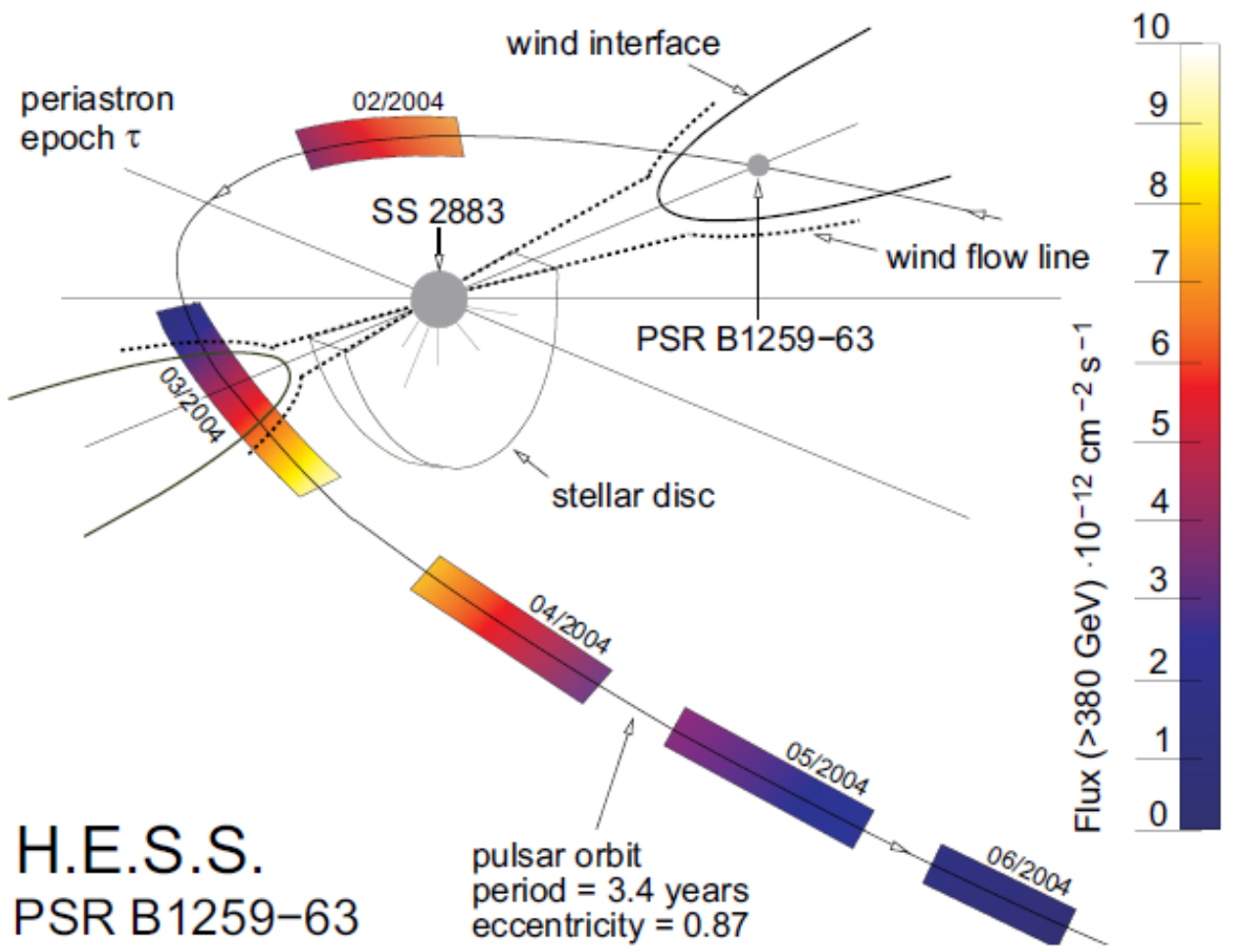

Fig. 13. Sketch of the orbit of PSR B1259-63 taken from 87. The color gradient bars indicate periods of H.E.S.S. observations and integral $\gamma$-ray flux from these observations.

radiation in the pulsar magnetosphere, produced by relativistic electrons trapped in extremely strong fields of neutron stars. Various models are proposed regarding the location of the region from where this radiation is emitted. This radiation could be originating from the region close to the neutron star surface as in polar cap scenario [96, 97, 98 or from farther out in the magnetosphere, as in slot gap [99,100,101 and outer gap [102,103,104] scenarios (see Fig. 15p). These models have different predictions about $\gamma$-ray spectra. So the detection of $\gamma$-rays above $10 \mathrm{GeV}$ was crucial to discriminate between pulsar emission models.

The previous generation IACTs did not detect any pulsed emission from Crab or any other pulsars even though several pulsars had been detected by space-based instruments like Fermi-LAT and earlier some of them were detected by EGRET onboard CGRO. Many of these pulsars, in fact, showed an indication of cutoff in their spectra at few GeVs. The first detection of pulsations from ground-based $\gamma$-ray telescopes was from Crab pulsar (period $\sim 33 \mathrm{~ms}$ ) at energies above $25 \mathrm{GeV}$ by MAGIC telescope [105]. Earlier observations with MAGIC had given a hint of pulsations above $60 \mathrm{GeV}$ [106. To verify this hint, observations were carried out with a new trigger system lowering the energy threshold from $\sim 50 \mathrm{GeV}$ to $25 \mathrm{GeV}$. Using 22.3 hours of data collected during October 2007 - February 2008, pulsations were detected from Crab at energies above $25 \mathrm{GeV}$ at a significance level of $6.4 \sigma$. Pulse profile from these observations is shown in Fig. 16 along with the profile obtained by EGRET. Main pulse $\left(\mathrm{P}_{1}\right)$ and interpulse $\left(\mathrm{P}_{2}\right)$ have similar amplitudes above $25 \mathrm{GeV}$ whereas at lower energies $(\mathrm{E}>100 \mathrm{MeV}) \mathrm{P}_{1}$ is dominant. Flux measured at $25 \mathrm{GeV}$ is much lower than extrapolation of the EGRET spectrum, indicating spectral cutoff between 


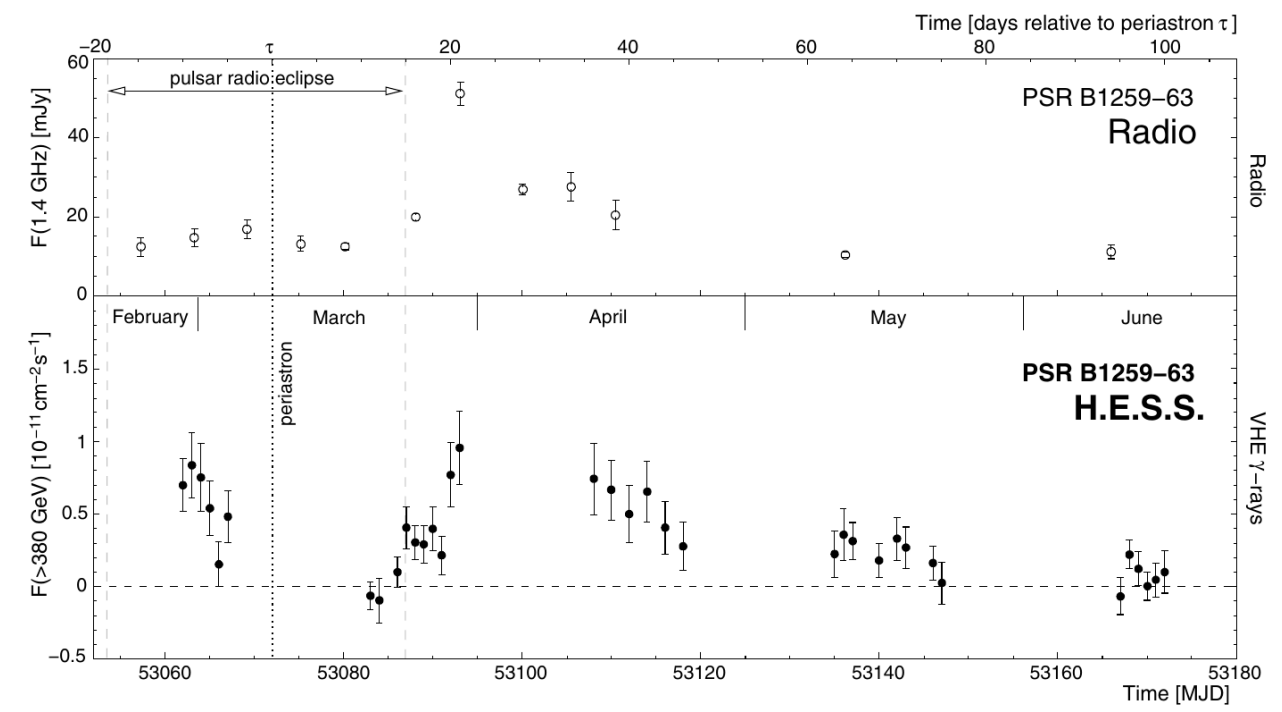

Fig. 14. VHE $\gamma$-ray and radio light curves of PSR B1259-63 around its periastron passage (epoch $\tau=0$ ) taken from 87 . The upper panel shows radio light curve for $1.4 \mathrm{GHz}$ from 88 with radio eclipse around periastron and the lower panel shows VHE $\gamma$-ray light curve from H.E.S.S.

5 and $25 \mathrm{GeV}$. Spectral cutoff energy depends on the maximum energy of electrons and absorption of emitted $\gamma$-rays in the pulsar magnetosphere. Considering the expected value of magnetic field strength for Crab pulsar, the sharp spectral cutoff is expected at relatively low energies, at the most few $\mathrm{GeVs}$, in the case of the polar cap model, due to magnetic pair production, compared to slot gap or outer gap models. So spectral measurements by MAGIC favoured outer gap and slot gap models over the polar cap model.

Later, based on the observations carried out during 2007-2011, VERITAS collaboration reported detection of pulsations at energies above $100 \mathrm{GeV}$ [107. Comparison of pulse profile from these observations with that detected by Fermi-LAT at lower energies revealed both $\mathrm{P}_{1}$ and $\mathrm{P}_{2}$ to be narrower by a factor of two or three. Also, the amplitude of $\mathrm{P}_{2}$ was found to be larger than $\mathrm{P}_{1}$, contrary to the trend seen at lower energies by Fermi-LAT. Gamma-ray spectrum over 100-400 GeV obtained by combining pulsed excess in $\mathrm{P}_{1}$ and $\mathrm{P}_{2}$ was well described by a power-law with index $-3.8 \pm 0.5_{\text {stat }} \pm 0.2_{\text {syst }}$. The spectrum over $100 \mathrm{MeV}-400 \mathrm{GeV}$, obtained by combining Fermi-LAT and VERITAS data is fitted with a broken power-law and it is statistically preferred over a power-law with exponential cutoff (see Fig. 17). The detection of $\gamma$-rays above $100 \mathrm{GeV}$ rules out curvature radiation as a possible production mechanism for these $\gamma$-rays considering a balance between acceleration gains and radiative losses by curvature radiation. This leads to two possible interpretations : a single emission mechanism other than curvature radiation dominating at all $\gamma$ ray energies or a second mechanism dominating above the spectral break. Further, MAGIC collaboration also reported spectral measurements of the pulsed component over the energy range of 25-100 GeV and found this spectrum to follow power-law, instead of exponential cutoff, after the break, ruling out the outer gap and slot gap models in their simplest version [109. In an extension of these measurements, spectra were fitted with a power-law over the energy range of 50-400 GeV indicating VHE emission could be an additional component produced by IC scattering of secondary and tertiary electron-positron pairs on IR-UV photons [110. There was also detec- 


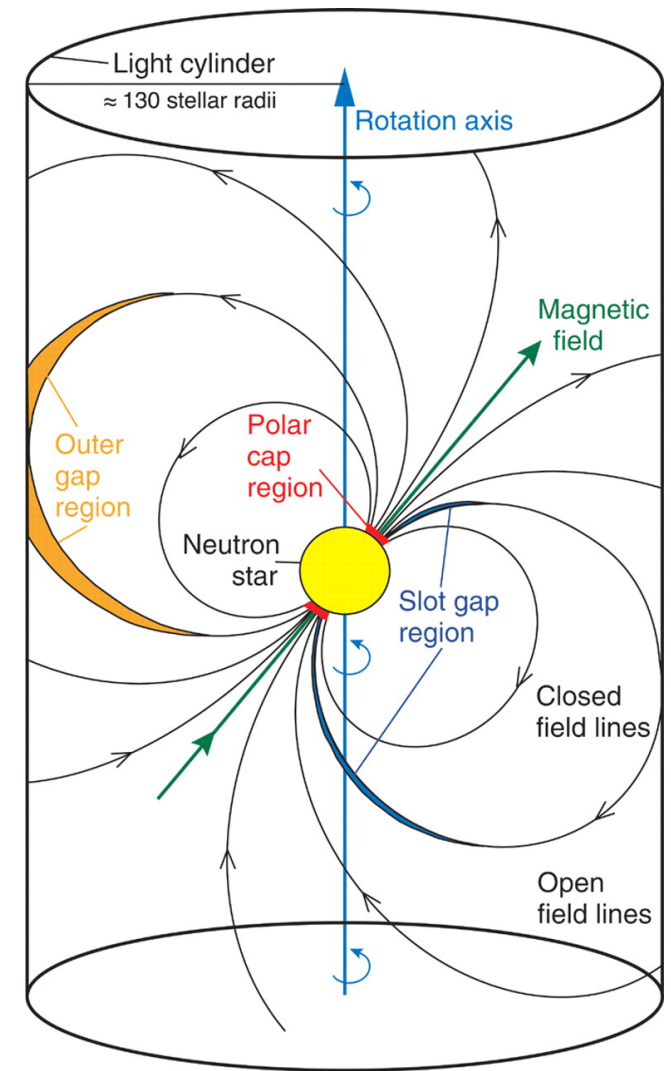

Fig. 15. Pulsar magnetosphere sketch taken from 105. depicting polar cap, outer gap and slot gap regions. Electrons which are trapped and accelerated along magnetic field lines emit radiation by the synchrotron-curvature mechanism. High energy $\gamma$-rays emitted get absorbed by magnetic pair production and photon-photon pair production. Magnetic pair production dominates close to the surface of the neutron star and generates a spectrum with super-exponential cutoff at few GeVs. Photon-photon collisions dominate in the outer magnetosphere and produce exponential cutoff in the spectrum at energies $>10 \mathrm{GeV}$.

tion of bridge emission above $50 \mathrm{GeV}$, with $6 \sigma$ significance level, between $\mathrm{P}_{1}$ and $\mathrm{P}_{2}$ 111. Finally, using larger data set spanning the period 2007-2014, MAGIC collaboration reported detection of pulsed emission upto $1.5 \mathrm{TeV}\left[112\right.$. Spectra of $\mathrm{P}_{1}$ and $\mathrm{P}_{2}$ were found to follow different power-law functions and join smoothly with Fermi-LAT measured spectra above $10 \mathrm{GeV}$. The presence of pulsations at TeV energies indicates the parent population of electrons with a Lorentz factor above $5 \times 10^{6}$, suggesting IC scattering as the emission mechanism and $\gamma$-ray production region in the vicinity of the light cylinder.

After successful detection of Crab pulsar at VHE $\gamma$-ray energies, several pulsars were observed by ground-based $\gamma$-ray telescopes. Pulsations have been detected so far only from two other pulsars : Vela and Geminga pulsar. Pulsed emission from Vela pulsar (period $\sim 89 \mathrm{~ms}$ ) was detected by $28 \mathrm{~m}$ diameter CT5 telescope of H.E.S.S.-II array in observations carried out during 2013-2015 [113. This is the largest IACT in the world and this is the first time VHE observations were carried out using groundbased telescope at sub-20 GeV energies. In these observations, pulsed $\gamma$-ray signal was detected at energies above $10 \mathrm{GeV}$ from $\mathrm{P}_{2}$ peak of Vela pulsar at a significance level of $15 \sigma$. The spectral shape was found to be consistent with that derived from 

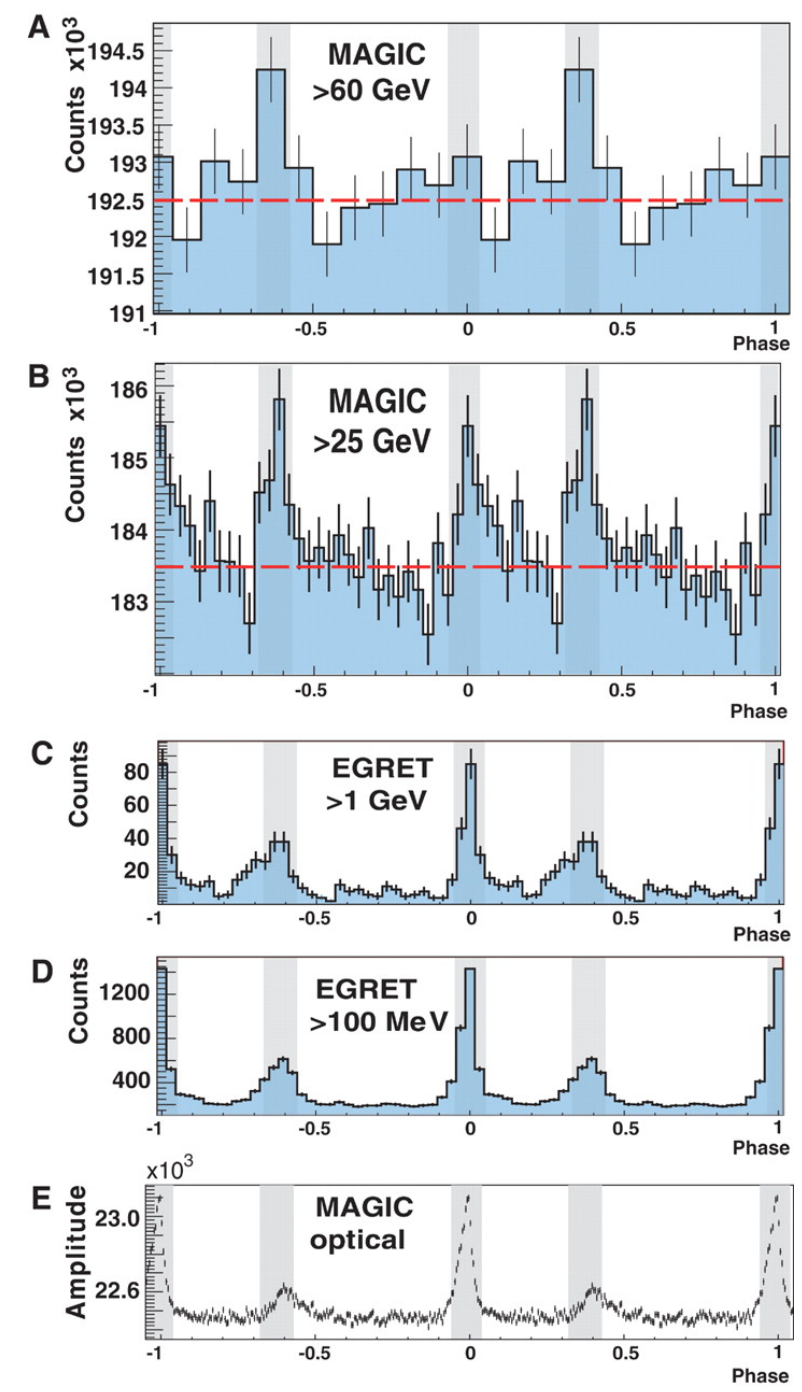

Fig. 16. Pulse profile of Crab pulsar in different energy bands taken from [105]. Panel A and $\mathrm{B}$ show evidence of pulsed emission above $60 \mathrm{GeV}$ and pulsations seen above $25 \mathrm{GeV}$ by MAGIC. Panel $\mathrm{C}$ and D show pulse profiles from EGRET at energies $>1 \mathrm{GeV}$ and $>$ $100 \mathrm{MeV}$ respectively. Last panel shows optical pulsation detected by MAGIC. Main pulse region $\mathrm{P}_{1}$ (phase : 0.94-0.04) and interpulse region $\mathrm{P}_{2}$ (phase : 0.32-0.43) are shaded.

Fermi-LAT observations. Combined spectral fit over 10-100 GeV showed curvature, confirming the sub-exponential cutoff found by Fermi-LAT. Also, weak evidence for emission above $100 \mathrm{GeV}$ was seen.

Pulsations were detected from Geminga pulsar (period $\sim 237 \mathrm{~ms}$ ) from MAGIC observations carried out during 2017-2019 [114. In this case, also, VHE emission was detected only from $\mathrm{P}_{2}$ peak at energies above $15 \mathrm{GeV}$ and the significance level of detection was $6.3 \sigma$. Spectrum from MAGIC observations was fitted with a single power-law with a steep index of $5.62 \pm 0.54$, smoothly extending the Fermi-LAT spectrum and ruling out exponential cutoff in joint energy range. Also, the sub-exponential cutoff was disfavoured at $3.6 \sigma$ level. A possible explanation of emission, in this case, 


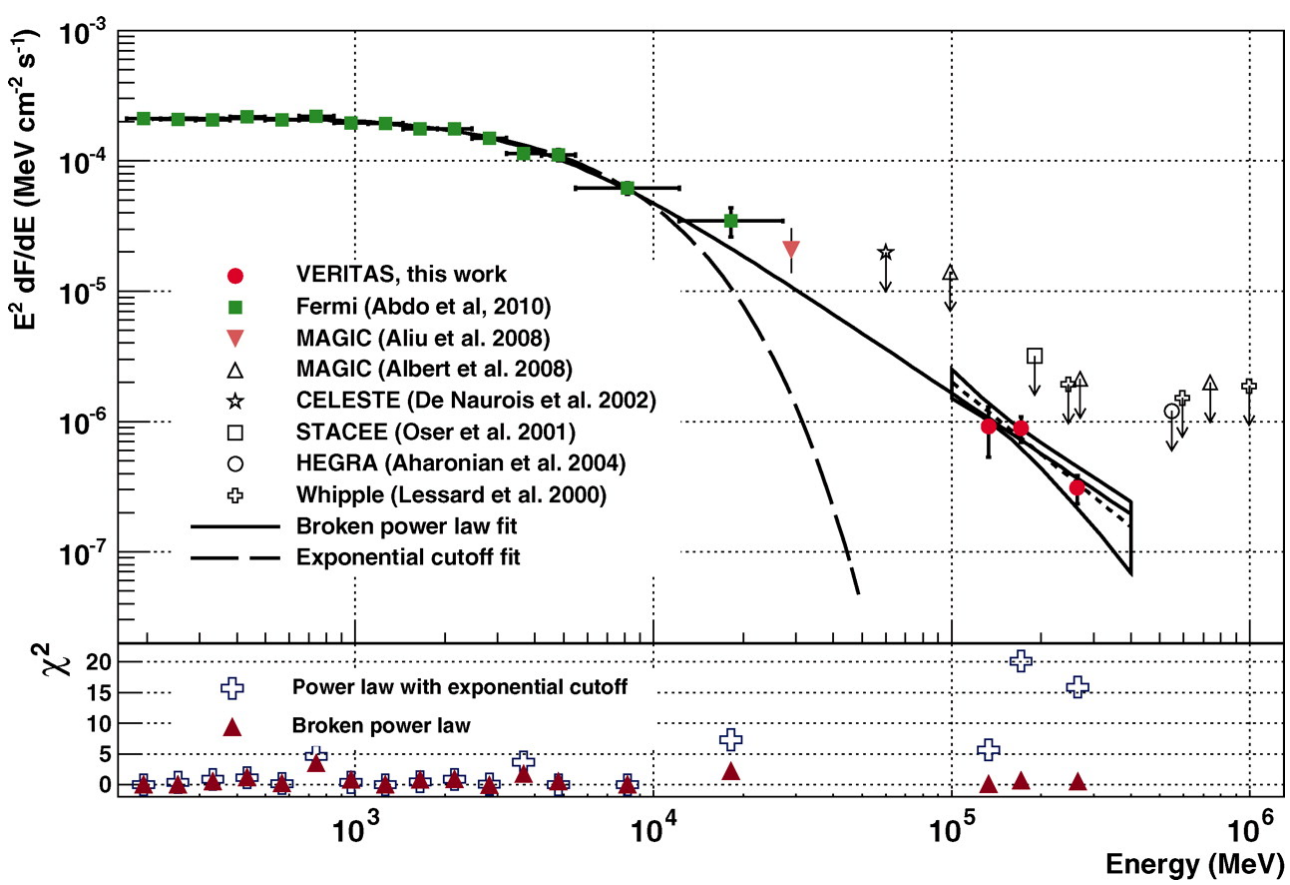

Fig. 17. $\gamma$-ray spectral energy distribution (SED) of Crab pulsar from 107 showing flux measurements from VERITAS [107], MAGIC ([105] and upper limit from [106]), Fermi-LAT 108 and earlier upper limits from various ground based telescopes. Solid line shows broken powerlaw fit to VERITAS and Fermi-LAT data whereas dashed line shows powerlaw with exponential cutoff. Bottom panel shows $\chi^{2}$ values for two models.

is given in terms of the outer gap model with pulsar viewed perpendicular to the rotation axis and emission originating from the northern outer gap. MAGIC energy range may correspond to the transition from curvature radiation to the IC scattering of particles accelerated in the northern outer gap.

\subsection{PeVatrons}

It is believed that sources such as SNR, PWN present in our galaxy are capable of accelerating cosmic rays (protons, heavier nuclei and electrons) to PeV energies, and therefore they can be classified as PeVatrons [115. When these cosmic rays interact with matter or photon fields in the vicinity of these sources, they produce very high energy $\gamma$-rays. These VHE $\gamma$-rays are expected to have $10 \%$ lower energy compared to parent cosmic rays, i.e. above 100s of TeV. Current IACTs have not detected any PeVatrons because of their limited sensitivity above a few tens of TeV. Air shower experiments, on the other hand, are suitable for the detection of PeVatrons, because of their wide field of view, better sensitivities above $100 \mathrm{TeV}$ and longer duty cycle.

Recently, LHAASO collaboration has reported [64] detection of $\gamma$-rays above 100 $\mathrm{TeV}$ from 12 galactic sources, including Crab nebula with statistical significance $>$ $7 \sigma$ (see Fig. 18). They have detected two ultra high energy (UHE) events from Crab [117. The first UHE event was detected on 11th January, 2020. The energy of this event was measured independently by two components of the array, square kilometer array (KM2A) and Wide-Field- of-view Cherenkov Telescope Array (WFCTA). The energy measured by KM2A was $0.88 \mathrm{PeV}$ and that by WFCTA was $0.92 \mathrm{PeV}$. The 
second event was detected on 4th January, 2021 measured only by KM2A as it was not in the field of view of WFCTA. The energy measured for this event was $1.12 \mathrm{PeV}$. This establishes Crab as a possible PeVatron. The broadband SED shown in the Fig. 19 is fitted with a one-zone model. Lower energy photons upto $\mathrm{MeV}$ energies are due to synchrotron emission by relativistic electrons, whereas higher energy photons with GeV-PeV energies are due to IC scattering of low energy photons. Above 100 $\mathrm{TeV}$ the $2.7 \mathrm{~K}$ cosmic microwave background radiation (CMBR) provide the main target photons for IC scattering. The best fit values for three free parameters for this model are power-law slope $(\alpha=3.42)$, cutoff energy $\left(E_{0}=2.15 \mathrm{PeV}\right)$ and magnetic field $(\mathrm{B}=112 \mu G)$. The KM2A spectral points from $10 \mathrm{TeV}$ to $1 \mathrm{PeV}$ are also fitted within statistical uncertainty by this one-zone model. However, the deviation in the range of $60-500 \mathrm{TeV}$ is $\sim 4 \sigma$. Spectrum seems to harden around $1 \mathrm{PeV}$. Under leptonic scenario, this can be explained by introduction of a second electron population. Even then the spectrum can not be fitted much beyond a few PeV. At higher energies, it is believed that $\gamma$-rays have a hadronic origin. Very energetic (multi-PeV) protons and atomic nuclei interact with the ambient photons or with gas/plasma in the ISM and produce charged and neutral pions. The $\gamma$-rays are produced from the decay of these secondary neutral pions. Charged pions, on other hand, produce neutrinos via decay.

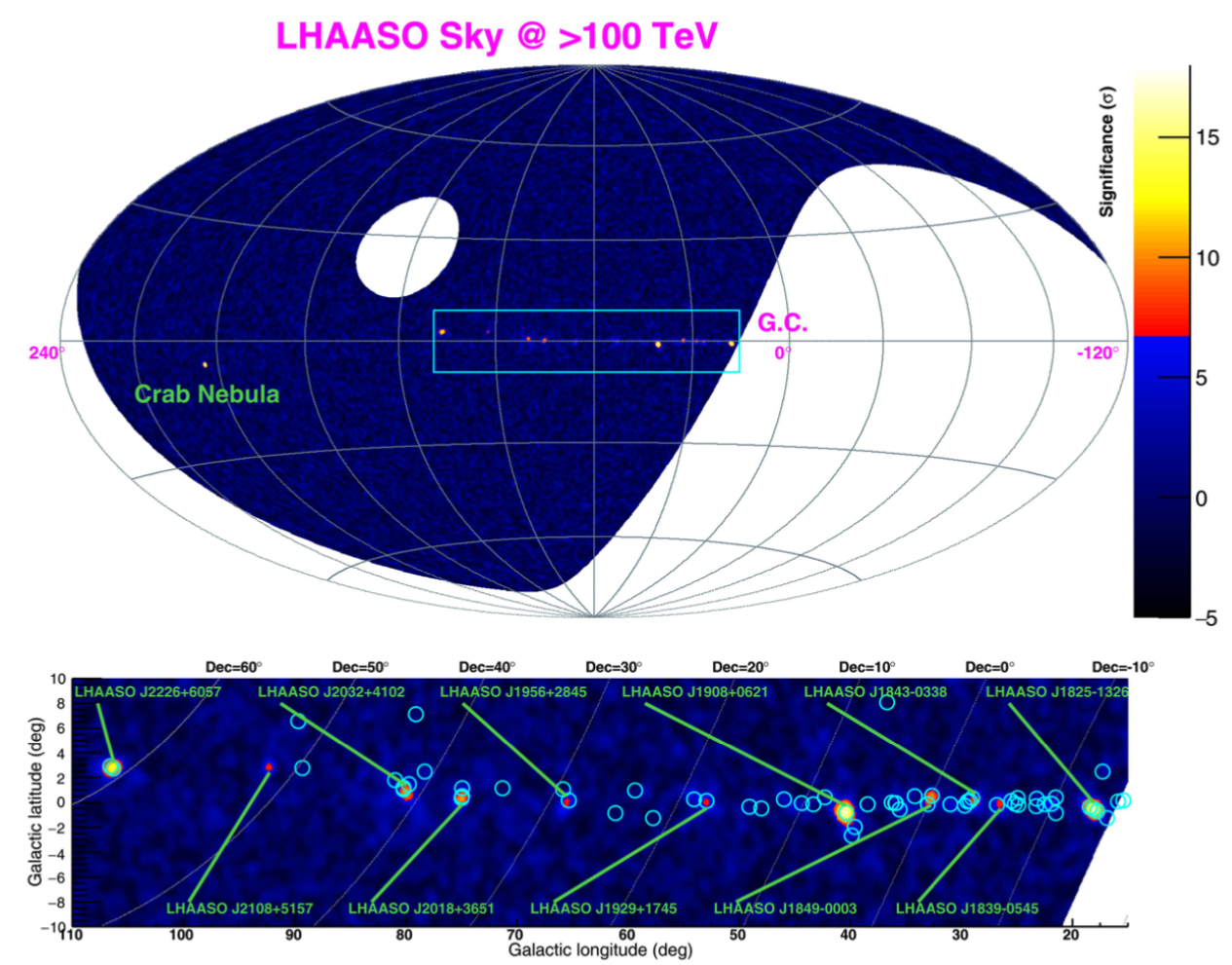

Fig. 18. Skymap of galactic sources detected by LHASSO above $100 \mathrm{TeV}$. Apart from Crab all others are located in the galactic plane. 64].

Except for Crab nebula, all other sources detected by LHAASO, are in the galactic plane. Many of these sources are extended upto $1^{\circ}$ (see Fig. 19). The three most luminous sources are LHAASO J1825-1326, LHAASO J1908+0621 and LHAASO J2226+6057. The spectral slopes of these sources steepen between $10 \mathrm{TeV}$ and 500 


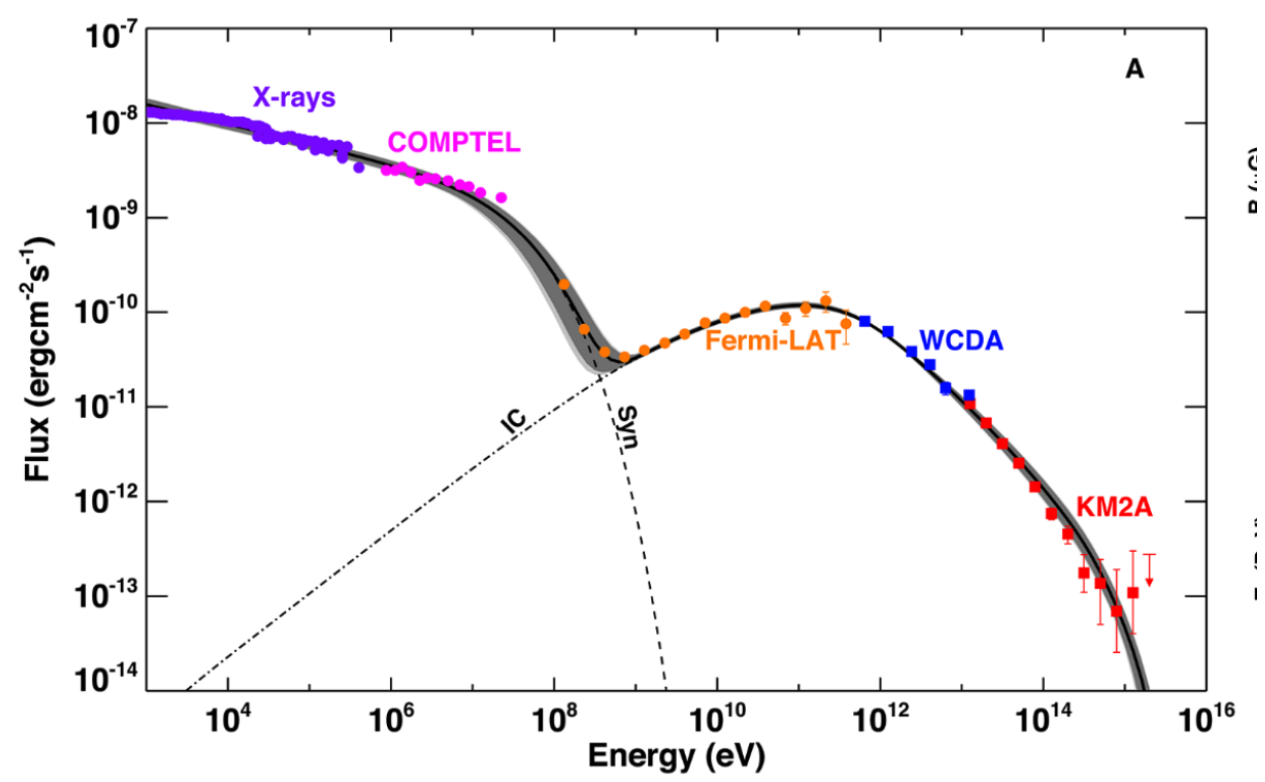

Fig. 19. The Spectral Energy Distribution of the Crab Nebula is fitted with one zone leptonic model. At lower energies curve is fitted with synchrotron emission and at higher energies radiation is fitted with IC scattering emission. [117.

$\mathrm{TeV}$, as $\gamma$-rays are likely to produce pairs as they interact with the interstellar radiation field and the CMBR. Possible candidates for PeVatrons are PWN, SNR and even young massive star clusters. PWNs are electron PeVatrons, where electrons are accelerated in the termination shock. Under a leptonic scenario, maximum energy achieved by IC process is a few PeVs, which means parameters are already stretched to their limits. At such high magnetic field IC becomes inefficient for $\gamma$-ray production due to Klein-Nishina effects. In view of these constraints with the leptonic scenario, it is believed that hadronic interactions might play a dominant role in $\mathrm{PeV}$ emissions. SNRs and young massive star clusters are the most favoured candidates for galactic cosmic rays, which can efficiently accelerate hadrons to $\mathrm{PeV}$ energies. The highest energy photon detected by LHAASO, coming from the source LHAASO J2032+4102, has the energy of $1.4 \mathrm{PeV}$. This source is believed to be associated with a massive young star cluster Cygnus OB2.

Prior to LHAASO, only handful of PeVatrons were detected by Tibet $\mathrm{AS}_{\gamma}, \mathrm{HAWC}$ and H.E.S.S. Tibet $\mathrm{AS}_{\gamma}$ has detected $\gamma$-rays above $100 \mathrm{TeV}$ from supernova remnant G106.3+2.7 [116. $\gamma$-ray emissions are believed to be coming from the molecular cloud surrounding the SNR. Tibet $\mathrm{AS}_{\gamma}$ data can be best fitted with hadronic model as protons can be accelerated to energies $1.6 \mathrm{PeV}$ by diffusive shock acceleration and then interact with molecular clouds and produce neutral pions. However, leptonic models are not ruled out, e.g. electrons can also be accelerated by reverse shock to very high energies at the SNR shell and produce $\gamma$-rays via IC scattering. This source was earlier detected by HAWC and VERITAS at lower energies. HAWC [118 data supports leptonic origin whereas VERITAS [119] data indicates hadronic origin for $\gamma$-ray emission.

HAWC has reported detection of $\gamma$-rays with energies $>56 \mathrm{TeV}$ from nine galactic sources including Crab nebula [56. Apart from Crab nebula rest are all located in the galactic plane. Three of these sources are detected above $100 \mathrm{TeV}$. One of the most interesting and well studied source in this list is the SNR, G106.3+2.7, which hosts the 
pulsar, PSR J2229+6114, known as the Boomerang pulsar, and nebula, also detected in radio, X-rays, $\mathrm{HE}$ and $\mathrm{VHE} \gamma$-rays. This region has been studied in detail using a joint analysis of VERITAS and HAWC data, which finds the spectrum extending upto $200 \mathrm{TeV}$ as a power-law. The proton cut-off energy is estimated to be $>800 \mathrm{TeV}$ which makes this source a possible Galactic PeVatron [118. It is argued that these $\gamma$-rays could have both leptonic and hadronic origin. One could use peak position of synchrotron emission in X-ray regime to distinguish between leptonic and hadronic origin, because in order to produce $100 \mathrm{TeV} \gamma$-rays via IC of CMB photons, electrons need to have energy around few hundreds of $\mathrm{TeV}$ and corresponding synchrotron peak will be at $10 \mathrm{keV}$.

H.E.S.S. has detected VHE emission above tens of $\mathrm{TeV}$ without a cut-off from a super massive black hole (SMBH) at the centre of our galaxy Sagittarius A [120]. This emission is coming from central molecular zone filled with gas where protons are accelerated to $\mathrm{PeV}$ energies. Leptonic scenario for this emission is ruled out because electrons will suffer radiative losses and hence IC scattering will not be very effective.

\section{Extragalactic Sources}

As mentioned in section 3 a large number of the sources detected at VHE energies by ground-based $\gamma$-ray telescopes are extragalactic, of which most are AGNs. VHE $\gamma$-rays are also detected from afterglow emission of a few $\gamma$-ray bursts (GRBs) and from a couple of starburst galaxies (SBGs). Cosmic rays with energies $>10^{15} \mathrm{eV}$ have Larmor radius greater than the size of the Milky Way, which means these charged particles cannot remain confined within the galaxy, and hence they are of extragalactic origin. They are likely to be produced inside the jets of AGNs, GRBs or SBGs.

\subsection{Active Galactic Nuclei}

AGNs are distant galaxies with very bright nuclei, with nuclear region outshining emission from the rest of the galaxy. AGNs are presumably powered by the accretion of matter from the host galaxy onto the supermassive black hole $\left(M_{B H} \sim 10^{6}-\right.$ $\left.10^{9} M_{\odot}\right)$ at the centre. Accreting matter, spiralling around the black hole, forms an accretion disk, which is surrounded by obscuring dusty torus (see Fig. 20). There are rapidly moving gas clouds, forming Broad Line Regions (BLR) and Narrow Line Regions (NLR). Finally, there are relativistic bipolar radio jets, presumably powered by accretion disk or spin of black hole [121,122.

The AGNs are largely classified as radio galaxies, quasars, blazars and Seyfert galaxies. According to the AGN unification model (see for example, [123, 124]), the underlying physics of AGN is the same and observed differences between various classes are due to differences in their orientation with respect to our line of sight. VHE $\gamma$-ray emission has been detected mostly from blazar class AGN and a few radio galaxies. Out of these two classes of AGNs, the blazars are a class in which the jets are viewed under a small angle with the line of sight, so we see Doppler boosted emission from these jets. Blazars are known to show variability in all wavebands on times scales from minutes to years. Also, there is a wide range of flux variation seen. The broadband SEDs of blazars typically show two broad peaks of non-thermal radiation, as shown in the Fig. 21. The low-energy hump lying between radio to X-rays is generally attributed to synchrotron emission from relativistic electrons present in the emission zone in the jet. However, the origin of $\gamma$-ray radiation in blazar jets, which forms the second hump of SEDs, is still a matter of debate. The emission mechanism could be either leptonic, where accelerated electrons and positrons produce the observed 


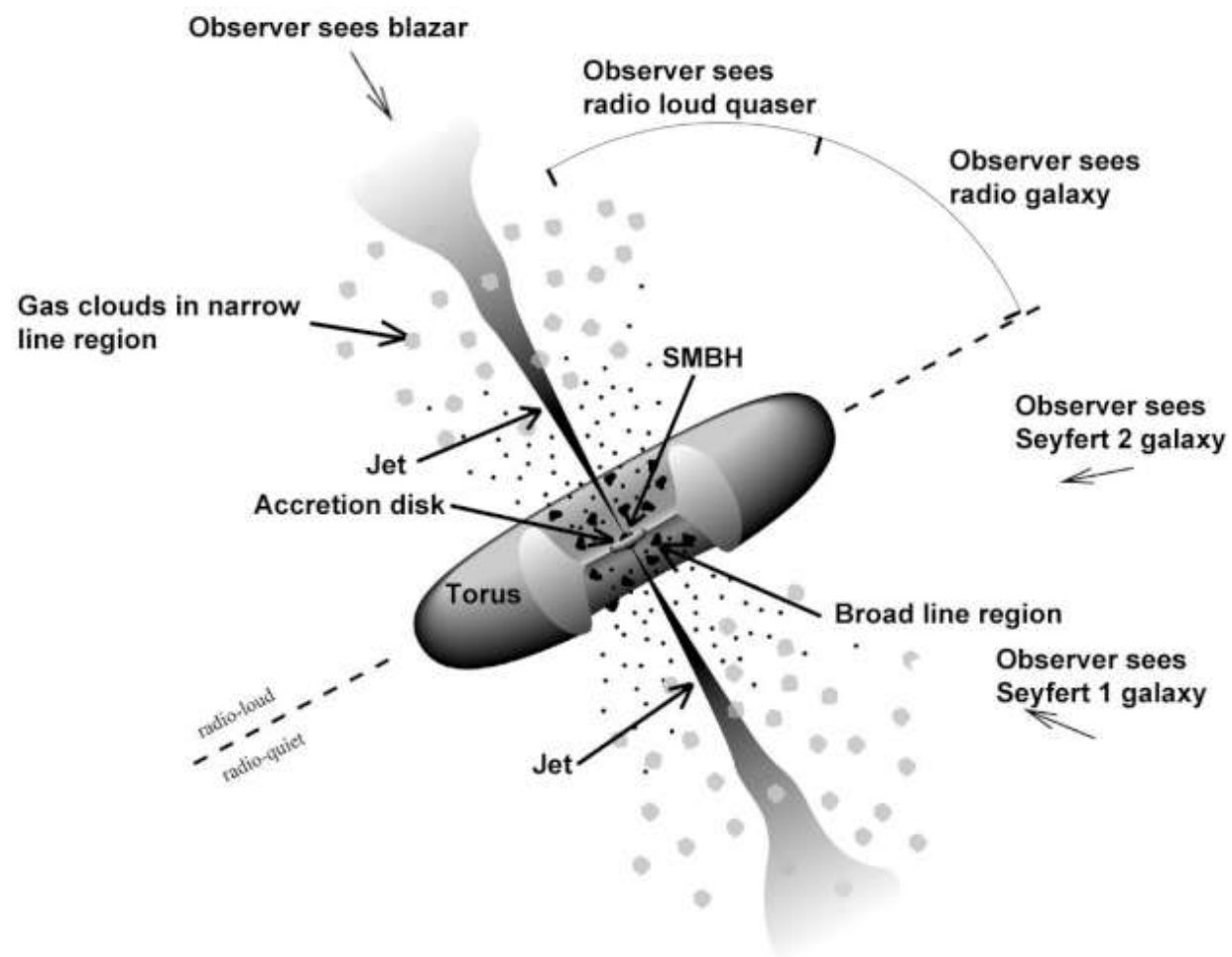

Fig. 20. Schematic representation of AGNs. According to the unified model, various classes of AGN correspond to their orientation with respect to the line of sight. Image credit : https://fermi.gsfc.nasa.gov/science/eteu/AGN/

emission through IC scattering of low energy photons, either from jet or from outside regions [125, or hadronic if accelerated protons and ions produce pions which decay into secondary electron-positron pairs, $\gamma$-rays, and neutrinos or protons producing synchrotron emission, or mixed [126, 127, 128, 129]. Moreover, hadronic emission is also required to explain the reported associations of blazars with high-energy neutrinos [130], thereby identifying blazars as possible sites for cosmic ray acceleration.

Further, blazars can be classified based on the presence of emission lines in their optical spectra as BL Lacertae objects (BL Lacs) and Flat Spectrum Radio Quasars (FSRQs). The BL Lac sources do not show strong emission lines in their optical spectra, whereas FSRQs' optical spectra show strong emission lines. The BL Lac type sources can be sub-divided into three subclasses based on the location of their synchrotron peaks in SEDs as Low frequency peaked BL Lacs (LBLs), intermediatefrequency peaked BL Lacs (IBLs), and high-frequency peaked BL Lacs (HBLs). The synchrotron peak $\left(\nu_{s y}\right)$ in LBLs has been observed at frequency $<10^{14} \mathrm{~Hz}$, IBLs have $\nu_{s y}$ between $10^{14} \mathrm{~Hz}$ and $10^{15} \mathrm{~Hz}$, whereas HBLs have $\nu_{\text {sy }}$ at $\geq 10^{15} \mathrm{~Hz}$. Also, an anticorrelation is seen between the location of the first peak and the source luminosity 131. As shown in Fig. 21, the first peak of SED moves from higher to lower frequency as we move from HBL to IBL and later to LBL and finally to FSRQ, while the source luminosity increases.

The jets of blazars are ideal laboratories to study the origin of high energy emission and the role of particle acceleration. But despite studies carried out on blazars, from various wavebands, there are several unanswered questions. For example, it is not 


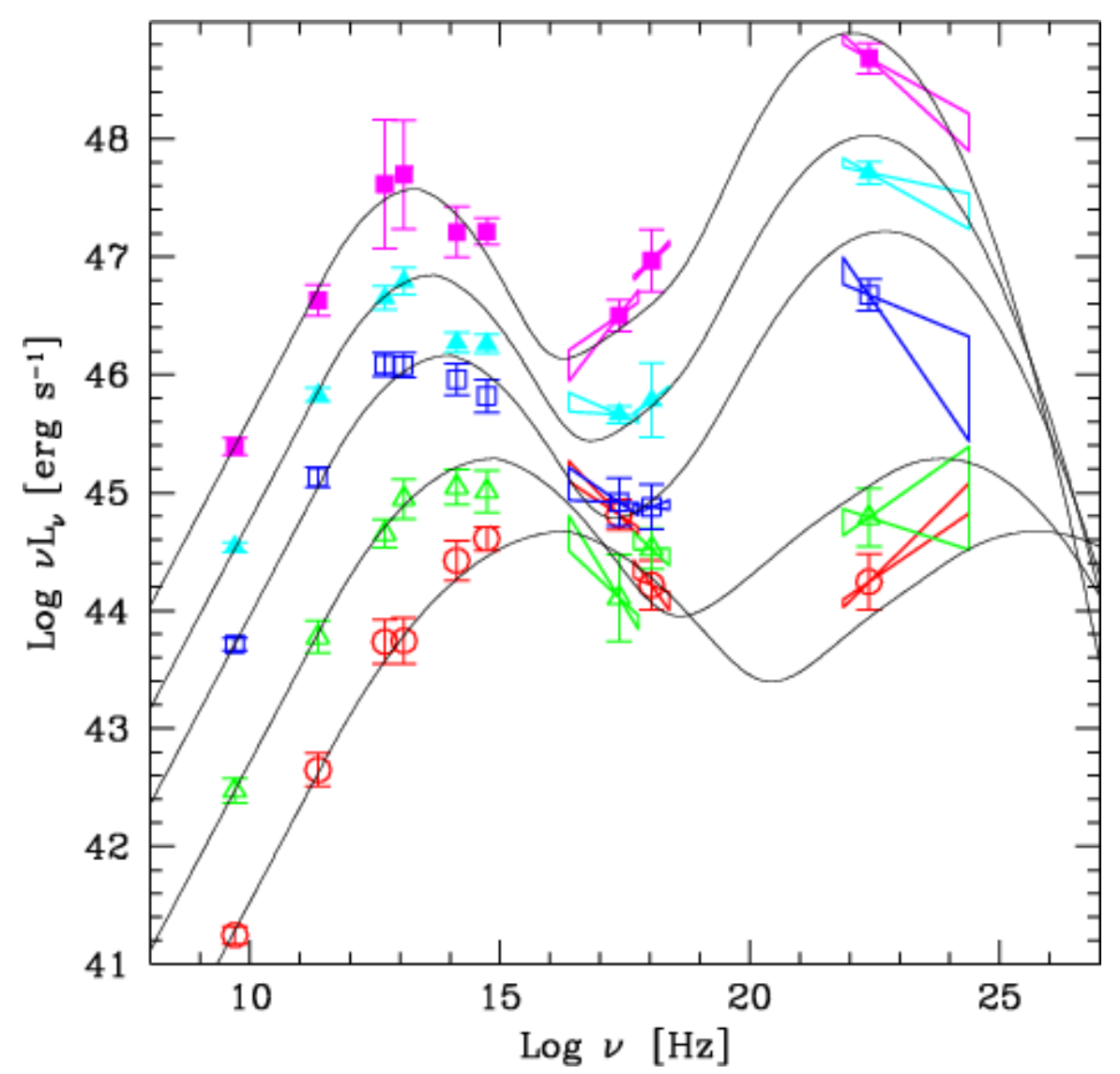

Fig. 21. Blazar sequence from 131] and 132. Anti-correlation is seen between location of the first peak of SED and the source luminosity (from magenta to red : FSRQ $\rightarrow$ LBL $\rightarrow$ IBL $\rightarrow$ HBL) Adapted from [132] with permission.

clear how exactly jets are launched and how they are collimated. Also, it is not known what is the mechanism for acceleration of particles in a jet, whether it is stochastic or diffusive shock acceleration or magnetic reconnection, or something else. Also, as mentioned earlier, it is unclear whether the high energy emission mechanism is leptonic, hadronic, or mixed. VHE $\gamma$-ray emission from blazars is expected to play a crucial role here and, along with multi-waveband data, may give valuable insight to understand the nature of the jets. The VHE $\gamma$-ray information is available in the form of temporal and spectral data, and using these, various studies are carried out as mentioned in subsections later.

Coming to VHE $\gamma$-ray observations, the extragalactic VHE $\gamma$-ray astronomy started three decades back with the detection of Mrk $421(z=0.03)$, by Whipple observatory for the first time [133. However, the second extragalactic source was detected only after five years, when Whipple detected a $\gamma$-ray signal from Mrk 501 134. Both these sources belong to HBL class of blazars. Currently, out of 77 blazars detected in VHE $\gamma$-rays by various telescopes, as per TeVCat 3 , more than $67 \%$ belong to the HBL class. The blazars of other classes such as IBL, LBL, and FSRQs were detected only after the present generation IACTs became operational during

${ }^{3}$ http://tevcat.uchicago.edu/ 
the century's first decade. The second-largest class amongst blazars detected at VHE energies is IBL with ten sources. One of the first detections of IBL class sources was from W Comae by VERITAS 135. However, the farthest VHE blazars are FSRQs. Currently, only eight FSRQs have been detected by IACTs, and the 3C 279 was the first one which was detected by MAGIC 136. Moreover, the farthest blazar detected as of now, S3 $0218+35(\mathrm{z}=0.954)$, is also an FSRQ.

\subsubsection{Temporal studies}

Independent of their subclasses, blazars are found to show variation of the flux on various time scales. During the brightest flare, the flux increases/changes by about an order of magnitude in some of the cases. In fact, there is a strong enhancement of flux variation in blazars due to Doppler boosting. The blazar emission is dominated by non-thermal radiation originating from a relativistic jet pointing towards Earth. The relativistic motion of the plasma boosts the non-thermal jet emission into a forward cone, and the emitted jet radiation is Doppler-boosted. As a result, the flux gets enhanced $\left(\propto \delta^{3}\right)$, and the variability timescales shortened (by $\propto 1 / \delta$ ) in the observer's frame. The flux from blazars varies over a variety of time scales from years to minutes.

Mrk 421 is one of the objects which is extensively observed by various atmospheric Cherenkov telescopes for last almost thirty years. Wide variation in flux is seen during this period, ranging from as low as $\sim 0.3$ Crab units to as high as $\sim 27$ Crab units. (In VHE $\gamma$-ray astronomy, as well as in many other branches of astronomy, flux is often mentioned in units of flux from Crab nebula, which is a steady source considered as a standard candle.) Several flaring episodes have been seen during this period. Fig. 22 shows the VHE light curve of this source during 1992-2008, obtained by combining count rate measurements from various telescopes like Whipple, CAT, HEGRA, H.E.S.S., MAGIC and VERITAS 137. Several flares can be noticed with flaring episode in 2001, when the flux level increased beyond 14 Crab units.

In addition to Mrk 421, another HBL, Mrk 501, has also shown remarkable flux amplitude variation when source flux changed from half to six times the Crab Nebula flux 138. Moreover, the HBL, 1ES 1959+650, has shown an "orphan" $\gamma$-ray flare as detected on 16th and 17th May 2002 by Whipple telescope [139, which was not accompanied by X-ray flare, as usually is the case for HBLs. After this bright flare, the source has not shown much activity until 2016, when exceptionally high flare measuring up to $\sim 3$ Crab units was seen by MAGIC on 13-14th June and 1st July 2016140.

Detection of blazars in their moderate and high states is quite common. However, detecting a blazar in a very low state could only happen with present-generation IACTs, which have pushed sensitivity to few $\%$ of Crab units in 50 hours of observation duration. Also, several faint blazars have also been detected, for example, HBLs like RBS0413 [141, 1ES 0414+009 [142, 1ES 1312-423 143. etc were detected at a flux level of as low as less than 1\% of Crab unit. Further, the VERITAS collaboration has observed an unusual flare from B2 $1215+30$. This flare was short and lasted only for a day. During this flare, the flux at other wavelengths, e.g., X-ray and optical, did not change [144. Similarly two other BL Lacs, PKS 1510-089 and PG 1553+113, have been detected in the state of low $\gamma$-ray activity [145, 146].

Most of the blazars show moderate flux variations on timescales of the order of a day, for example, Mrk 421 [147,148. Whereas, hour and sub-hour scale flux variations are less common and only detected during the high flux states, e.g., Mrk 421 [149], Mrk 501 [150, 151,152, PKS 1510-089 [153] and BL Lac [154]. During some of the flares, the flux doubling/halving times down to few 10's of minutes are seen, for example, 


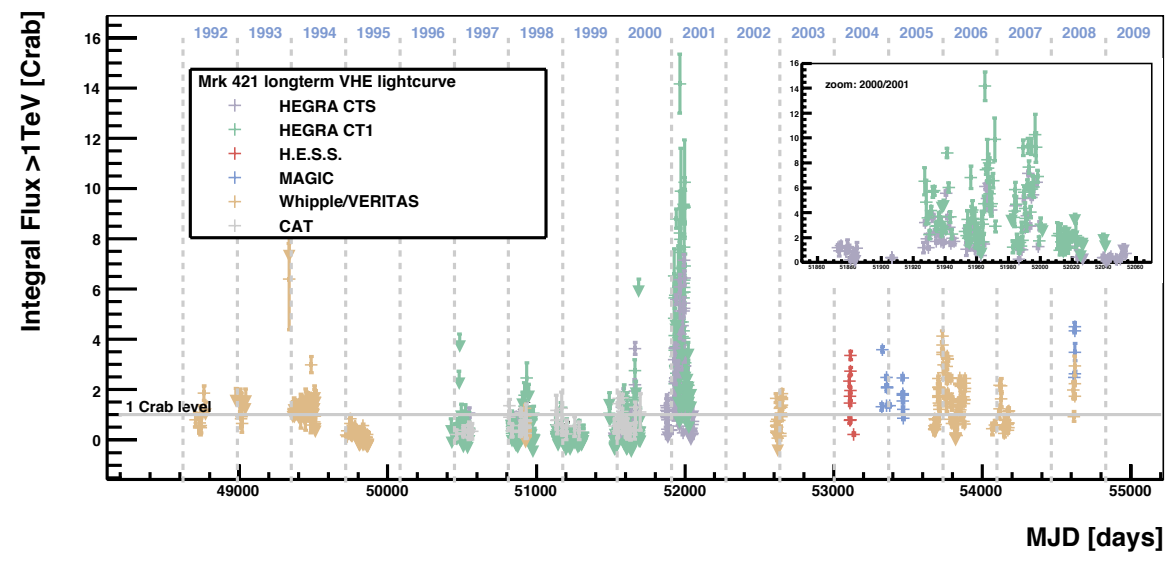

Fig. 22. Long term lightcurve of Mrk 421 during 1992-2008, obtained by combining data from various telescopes and normalizing to energy threshold of $1 \mathrm{TeV}$. Inset shows zoom over a period of strong activity in 2000/2001. Adapted from [137] with permission.

16 minutes time scale was observed in Mrk 421 155. Also the decay time scale of $13 \pm 4$ minutes was seen in one of the IBLs, BL Lac [154. The most remarkable and unprecedented flux variations on the timescales of a few hundred seconds were observed from HBLs Mrk 501 156 and PKS 2155-304 157. Fig. 23 shows one minute binned HESS light curve of PKS 2155-304 above $100 \mathrm{GeV}$ during a very bright and remarkable flare in 2006 [157]. The light curve clearly shows substructures in the flare, 
suggesting that the burst was composed of a few rapid flares of the order of a few minutes. The flux changes from 0.65 to 15 Crab units, and the fastest flux doubling time was 200 seconds during this activity period. Other remarkable flares from Mrk $501[156$ and Mrk 421 [158 are discussed later in this article. Out of the eight FSRQs detected at VHE energies, some have shown significant variability. The FSRQ, PKS $1441+25(\mathrm{z}=0.940)$, has shown the variability on the timescale of a week [159]. Whereas, the FSRQ, PKS $1222+21$, was found to be variable on sub-hour time scales by MAGIC [160.

The observed day-scale to hour-scale variability from blazars, at VHE energies, is consistent with the prevailing paradigm that variability should be imprinted on the scale of the size of the event horizon of the black hole or passing/standing shock in the jet. It is assumed in the internal shock (shock-in-jet) model that different parts of the jet moving at different speeds may collide and give rise to the shock front. The shock front may accelerate particles to high energies and dissipate energy in the form of non-thermal radiation. It is assumed that a shock extends over the jet's entire diameter.

Though the observed day-scale variability is consistent with the shock acceleration scenario, the observed rapid variability (of the order of a few minutes to sub-hour scales) has challenged this scenario. Also, observed variability is too short for originating directly from the central engine, as it is smaller compared to event horizon light-crossing time, which is typically around 80 minutes for black hole mass of $10^{9}$ $M_{\odot}$. Moreover, this situation gets even more dramatic after the detection of fast flares and high energy photons from FSRQs during the flares [160, as spherical shell BLR of FSRQ becomes opaque to $\gamma$-rays above $20 \mathrm{GeV} /(1+\mathrm{z})$ due to $\gamma-\gamma$ interaction [161. The high energy $\gamma$-ray photons which are emitted within the BLR, at the distance of $\sim 0.1 \mathrm{pc}$ from the central engine, are expected to be absorbed by the UV photons emitted by $\mathrm{H}-L y_{\alpha}$ and continuum emission of a quasar with an accretion disk luminosity above $10^{45} \mathrm{ergs} / \mathrm{sec}$. The observed rapid variability warrant more sophisticated scenarios, e.g., turbulence, multi-zone emission, or magnetic reconnection, emission from the magnetosphere of black holes [162, 163, 164, 165.

Multiwaveband light curves of blazars is another useful tool to get insight into emission mechanisms. Several observation campaigns have been carried out to study blazars in flare as well as low state of activity, spanning various wavebands. Fig. 24 shows one example of Mrk 421 multiwaveband light curves extending from radio to VHE $\gamma$-rays, from observation campaign carried out in April 2013, when the source was detected in flaring state. These light curves are searched for correlated variability. Correlated variability in X-ray and VHE $\gamma$-ray bands has been seen in HBLs on several occasions. One of the early results, for example, is from monitoring campaign of Mrk 421 during 2003-2004 involving VHE data from Whipple telescope and X-ray data from RXTE-ASM which showed correlated X-ray and VHE $\gamma$-ray variability during an outburst of 2004 [166. Later this kind of correlated variability between X-ray and VHE bands was seen in many sources, for example, HBLs like Mrk 421 [167,168, 148, 149], Mrk 501 [152, 169, 170], 1ES 2344+514 [171] 1ES 1959+650 [140, PKS 2155-304 [172. These correlations have been seen in flare as well as low to moderate flux states on several occasions. These are either checked through X-ray vs VHE $\gamma$-ray flux plot or through discrete correlation function (DCF). One example of DCF between VHE $\gamma$-ray and X-ray lightcurves, as a function of time lag in days is shown in Fig. 25. Correlation is seen between these bands with zero lag and corresponding DCF value is about $0.76 \pm 0.1$ [140]. These correlations indicate a similar origin for X-ray and VHE $\gamma$-ray photons, which we will see further in discussion on multiwaveband spectral results. There are also occasions when such correlations were not seen, for example, in Mrk 421 [173,147], Mrk 501 [151] and PKS 2155-304 [174]. 
Also as mentioned earlier, orphan flare was seen from 1ES19159+650 on one occasion, when VHE flare was seen without accompanying increase in X-ray flux.

One interesting result is obtained from data studied during historical flare from Mrk 421 in February 2010, when VERITAS detected source at the flux level of $\sim 27$ Crab units at the peak of the flare, above $1 \mathrm{TeV}$ [158]. During this flare, simultaneous optical data was recorded using $1.3 \mathrm{~m}$ Robotically Controlled Telescope (RCT) located at Kitt Peak National Observatory, giving high-cadence optical exposure in the form of 2-minute exposures in the $\mathrm{R}$ band. Fig. 26 shows VERITAS light curve along with $\mathrm{R}$ band light curve. DCF analysis of these light curves shows evidence for an optical lag of $\sim 25-55$ minutes with respect to VHE flux. This is the first time, correlation is reported between these bands on such short time scales.

Another type of study carried out with multiwaveband temporal data is the wavelength dependence of variability. The variability is quantified in terms of flux variance intrinsic to the source, normalized to mean flux. This is called fractional variability $\left(\mathrm{F}_{\text {var }}\right)$ and is given by

$$
F_{v a r}=\sqrt{\frac{S^{2}-\left\langle\sigma_{e r r}^{2}\right\rangle}{\langle x\rangle^{2}}}
$$

where $S$ is the standard deviation of the flux measurement, $\left\langle\sigma_{e r r}^{2}\right\rangle$ the mean squared error and $\langle x\rangle^{2}$ the square of the average photon flux (see [175] for example). In case of blazars, overall trend of increase in $F_{v a r}$ with energy is seen. This trend is observed quite often, with maximum variability at VHE energies, in Mrk 501 [151, 152, 176] and in case of Mrk 421 [177, 178. Fig. 27 shows one typical example of $F_{v a r}$ as a function of energy for Mrk 501, for multiwaveband observations carried out in 2009 151. Similar trend is noticed in some other blazars like MAGIC J2001+439 [179]. However, on some occasions, different trend showing two hump structure, with $F_{\text {var }}$ peaking in X-ray and later in VHE $\gamma$-rays is also seen, for example in Mrk 421 [180, 181, 182. This is similar to the two-humped broad band SED, which is described later, and explained in terms of SSC model [181]. However, more sensitive observations are required to put constraints on the emission models from $\mathrm{F}_{\text {var }}$ studies.

Apart from these studies, the power spectral density (PSD) is another important tool used for characterizing variability. It provides a measure of power present at different timescales in a light curve. Though PSD is used extensively on wavebands like optical or X-ray, such studies are less common in the VHE band due to gaps in the data. Such studies have been carried out on the $\sim 9$ years of H.E.S.S. observations of PKS 2155-304 [183. The observed variability during the quiescent state is consistent with flicker noise (slope of the power-law $\beta \sim 1$ ). Whereas, the variability in the flaring state during 2006 is well described by red noise $(\beta \sim 2)$ in the VHE band.

\subsubsection{Spectral Studies}

The origin of $\gamma$-ray radiation in blazar jets is still a matter of debate. This is due to the fact that, both, the emission mechanism and the location of the emission region in the jet, is unclear. As mentioned earlier, the emission mechanism could be leptonic, hadronic or mixed. The association of blazars with high energy neutrinos requires explanation in terms of hadronic mechanisms. The prevalence of the leptonic and hadronic emission processes reflect the physical conditions of the plasma where the particles get accelerated and thus depend on the location of this region in the jet.

Most of the TeV blazars show $\gamma$-ray peak around HE $\gamma$-rays, and the observed spectrum above $100 \mathrm{GeV}$ may be described by power-law with the index of 3-4 (as per TeVCat). However, sometimes even harder spectral indices are also detected such 
as index of about 2.2 in case of Mrk 421 [167, $\sim 2.7$ for Mrk 501 184 and $\sim 2.7$ for $1 \mathrm{ES} 1959+650$ [185. Whereas steep spectrum with index $\sim 4.8$ has been detected in HBL, PG 1553+113 186. Also, one IBL, 3C66A, was detected in flaring state with a steep spectrum (index 4.1) [187.

In order to get insight into emission mechanisms, multiwaveband spectral studies have been carried out for various blazars, conducting multiwaveband campaigns (MWC). A large fraction of blazars detected in VHE $\gamma$-rays are HBLs. One prominent example is Mrk 421. Fig. 28 shows multiwaveband SED of Mrk 421 from observations carried out during 19 January - 1 June 2009 [147, including data from radio, optical, UV, X-ray, HE and VHE $\gamma$-rays. It shows a characteristic double-peaked profile, with the first peak in the X-ray band and the second one in $\gamma$-rays. Fig. 29 shows this SED fitted using simple leptonic model, SSC. This model assumes a spherical blob of plasma, inside the jet, moving towards the observer, travelling with a bulk Lorentz factor. The emission volume is assumed to be filled with an isotropic population of electrons and a randomly oriented uniform magnetic field. The energy spectrum of injected electrons is given by a broken power-law (see for example, [139]). These energetic electrons emit synchrotron photons producing the first hump in SED. These synchrotron photons are then upscattered to higher energies by the same population of electrons forming the second hump in SED. The emission is Doppler boosted and the size of the emission zone is related to variability time scale $\left(t_{v a r}\right)$ by light-travel time argument. Apart from reproducing typical HBL SED, this model also explains the correlated variability seen between X-ray and VHE bands earlier.

In many cases, SEDs of HBLs have been explained in terms of simple one-zone SSC model, e.g. Mrk 421 [166, 188, 171, 155, 189, 180,178] etc, Mrk 501 [190, 156, 184, 169] etc, 1ES1959+650 139,191,192, 1ES2344+514 193,171] and many other sources. In several cases, 'harder-when-brighter' trend i.e. hardening or flattening of the spectrum with an increase in flux is seen at X-ray and VHE $\gamma$-ray energies. Also, both the peaks of the SED are found to shift to higher energies as the source brightens, on several occasions. However, during one of the MWCs of Mrk 501 the X-ray peak was found to shift by over two orders of magnitude in photon energy between the two flux states while the VHE peak varied little [184. Probably this limited shift in the VHE peak may be explained by the transition to the Klein-Nishina (KN) regime. However, during 2012 MWC the high energy peak shifted to the highest energies of $\sim 2 \mathrm{TeV}$, which is seen for the first time in any $\mathrm{TeV}$ blazar. During this MWC, the highest activity occurred on 9th June 2012, when the VHE flux was $\sim 3$ CU, and the peak of the high-energy spectral component was at $\sim 2 \mathrm{TeV}$. During this MWC both the X-ray and VHE $\gamma$-ray spectral slopes were measured to be extremely hard, with spectral indices $<2$ during most of the observing campaign, regardless of the X-ray and VHE flux. This was the first time an HBL, Mrk 501, behaved like an extreme high-frequency-peaked blazar (EHBL), throughout the 2012 observing season 152. These types of shifts are seen in some other objects as well. For example. 1ES $2344+514$, was also found to change its state from HBL to EHBL as seen by FACT and MAGIC telescopes 194. One of the IBLs, 1ES 1215+303, has shown an extreme shift of synchrotron peak from optical to X-ray (IBL to HBL) [195].

Another interesting spectral feature was observed from Mrk 501, a narrow feature at $\sim 3 \mathrm{TeV}$, in the VHE $\gamma$--ray spectrum measured on 19 July 2014 during the MWC, with more than $3 \sigma$ confidence level [176. This feature is seen in Fig. 30 , the top panel shows the observed spectrum, whereas the bottom panel shows the spectrum corrected for absorption by extragalactic background light (EBL). Spectral fits with log-parabola (LP) and combination of LP with strongly curved LP i.e elogpar or EP, are shown. Several explanations are given for this feature, including a pileup in electron energy distribution (EED), two SSC regions in jet with one dominated by narrow EED, emission from an IC pair cascade from electrons accelerated in mag- 
netospheric vacuum gap etc. One of the scenarios here involves two SSC regions in jet. The one-zone SSC model, though successfully applied in many cases as discussed above, could be a simplified picture. There could be multiple emission regions in the jet. In some of the cases, SEDs are fitted using a two-zone SSC model. One such example is shown in the Fig. 31 [196]. In this figure, SED of Mrk 501 in a moderately high state in April-May 2011 is modelled in terms of SSC emission from two zones, inner zone responsible for VHE emission and outer zone contributing to HE $\gamma$ ray emission. Similar two-zone SSC models have been used to explain SEDs for several other observations of Mrk 501 [151, Mrk 421 [197.

So far we have considered mostly HBLs, where SEDs can be explained in terms of SSC model. As we move to IBLs and later to FSRQs, there are other sources of seed photons available for inverse Comptonization and they dominate synchrotron photons. These are the photons provided by radiation fields external to the jet, for example, originating from reprocessing of UV photons from accretion disks, from BLR [125], or, for the emission regions at larger distances from the black hole, it could be diffuse IR radiation from dusty torus [198. Fig. 32 shows example of SED of FSRQ 3C279, from observations carried out in June 2015 during flare [199]. SEDs shown in the figure correspond to various stages of flare and pre-flare states. These are fitted with a combination of SSC and EC i.e. external Compton. EC consists of three components, IC scattering of photons from the accretion disk, BLR and dusty torus, with BLR component dominating. The combination of SSC and EC is used to explain SEDs in many other cases, e.g. FSRQs like PKS 1510-089 200,201, PKS 1222+216 202], PKS 1441+25 [159] etc, IBLs like W Comae [135,203] and on few occasions even for HBLs, e.g., 1ES1959+650 [204], RBS0413 [141], 1ES 1440+122 205 etc.

Apart from using leptonic models to explain blazars' SEDs, there have been several attempts to provide an explanation in terms of hadronic models. As AGNs are likely sites for the acceleration of cosmic rays at energies above $10^{15} \mathrm{eV}$, hadronic models provide support to this scenario. One such example is shown in Fig. 33 where SED of HBL, 1ES1959+650, is fitted using hadronic model 140. Here, it is assumed that relativistic protons are injected in the emission region, in addition to leptons. Synchrotron emission from these protons is able to reproduce the second peak of SED satisfactorily. There is also a photo-meson component arising from the interaction of high-energy protons with low-energy synchrotron photon field, though it is not a dominant contribution. To account for observed variability time scales of $\sim$ an hour, a magnetic field of about 100 Gauss is assumed in contrast to about a fraction of a Gauss normally used in leptonic models. A similar hadronic model was used earlier to explain TeV spectrum of Mrk 501 during an extraordinary flaring episode seen in 1997 [126]. Some more examples of usage of hadronic models are for 1ES2344+514 [194] and Mrk 501 [147. On several occasions lepto-hadronic models are also used. In this case, second peak is explained as a combination of SSC and photo-meson cascade component. Examples of lepto-hadronic models are 1ES19159+650 [140], Mrk501 [184, RXJ0648.7+1516 [206], RBS0413 [141], 1ES 0414+009 207] etc. Both types of models, hadronic and lepto-hadronic, predict neutrino emission.

\subsubsection{Multi-messenger Studies}

The most direct proof for the acceleration of cosmic rays in AGNs can come from the detection of neutrinos from these objects. Detection of a high-energy neutrino by IceCube detector on 22 September 2017 and, its coincidence, in direction and time, with the blazar TXS $0506+056$, is an important result. The neutrino was detected with more than $3 \sigma$ confidence level to be coincident with the blazar [208]. 
This detection indicates the blazar TXS $0506+056$ to be only the third astrophysical source to be identified after the Sun and a nearby supernova $1987 \mathrm{~A}$ as a neutrino emitter. TXS 0506+056, was undergoing a $\gamma$-ray flare during the neutrino detection by IceCube. This prompted a multi-instrument/telescope campaign to observe the source. The IceCube collaboration also found an excess lower-energy neutrino emission at the location of the blazar in the previous several years [209]. In addition to TXS 0506+56, a high-fluence blazar PKS B1424-418 has also been found in the outbursts during a PeV-energy neutrino event from the same direction 210. These kinds of multi-messenger studies are likely to provide crucial information about emission mechanisms in blazars as well as cosmic ray acceleration.

\subsection{Radio galaxies}

The radio galaxies have provided vital information about the location of the emission region of the $\gamma$-rays in AGNs. Observations of radio galaxies IC 310 and Cen A have shown that $\gamma$-ray emission in these galaxies might originate very close to the black hole or far from the black hole. MAGIC has reported rapid variability with doubling time scales faster than $5 \mathrm{~min}$ from IC 310. Based on the causality argument this constrains the emission region to be much smaller than the black hole event horizon. In this case, the emission could be associated with a pulsar-like particle acceleration in the electric field across a magnetospheric gap situated at the base of the radio jet 211. The recent observations of another radio galaxy, Centaurus A, from the H.E.S.S. telescope have resolved its large-scale jet at TeV energies. These observations suggest that a major part of the VHE $\gamma$-ray emission arises far from the central engine, around $2.2 \mathrm{kpc}$ [212]. Other radio galaxies detected by different IACTs are M87 [213] and 3C264 [214]. M87 did not show any increase at radio wavelengths but enhanced X-ray flux was observed during the 2010 VHE flare [215]. A strong VHE $\gamma$-ray flare accompanied by an increase of the radio flux from the nucleus of M87 was seen during 2009, suggesting that the charged particles are accelerated to VHE energies near the black hole. The high-resolution radio observations combined with VHE could locate the site of $\gamma$-ray production [216].

\subsection{Gamma-ray Bursts}

The Gamma-Ray Bursts (GRBs) are the most violent explosions in our Universe. They usually release a huge amount of energy $\left(10^{51}-10^{54} \mathrm{erg}\right)$ in a very short time [217,218. The light curves of these bursts are characterised by their rapid and irregular variability on timescales of milliseconds to thousands of seconds. The amount of energy released by GRBs in such a short duration, known as prompt emission, is equivalent to the total energy released by the Sun during its entire lifetime, in less than a second. The photons emitted during the prompt phase are in the energy range of $\mathrm{keV}-\mathrm{MeV}$. GRBs are distributed isotropically in the sky, which indicates that these sources are extragalactic, located outside the Milky Way. Also from the total energetics and rapid variability, it was evident that these catastrophic events are of stellar origin. The prompt emission is followed by a long duration afterglow emission across the electromagnetic spectrum, spanning from radio to higher energies, even as high as VHE $\gamma$-rays in some of the cases. The key properties of GRBs, such as exact locations, nature of the progenitor etc are determined from the afterglow observations.

GRBs are divided into two classes. If the prompt emission lasts for less than 2 seconds, GRBs are classified as short GRBs. If durations are longer than 2 seconds, 
then they are classified as long GRBs. Short GRBs are believed to be due to mergers of compact objects, e.g. neutron star - neutron star merger or neutron star - black hole merger. Long GRBs, on the other hand, are associated with the death of massive stars or core-collapse supernova. In both cases, a black hole is formed and a relativistic jet emerges from the central engine.

The prompt emission arises from internal shocks. An enormous amount of energy is released during the burst in a short time interval from a very compact region. As a result, radiation pressure overcomes gravity and heats up matter into a fireball. The fireball then expands relativistically by this radiation pressure. The matter (electron, positron, proton, neutrons, photons) is ejected in successive shells. Shells move with different speeds, internal shocks are produced when slow-moving shells collide with fast-moving shells. After some time, the fireball becomes transparent and emits radiation. Synchrotron radiation produced by electrons escape at this phase, observed as prompt-emission. The prompt emission is followed by a long afterglow emission when relativistic jet interacts with the ambient medium (see Fig. 34).

According to various theoretical models, VHE $\gamma$-rays are expected from afterglow emission $219,220,221$. Under the leptonic scenario, there are two possibilities for VHE $\gamma$-ray emission; synchrotron emission by electrons in the local magnetic field or via the SSC mechanism. If $\mathrm{VHE} \gamma$-rays are produced by synchrotron emission by electrons, then electrons need to be accelerated to $\mathrm{PeV}$ energies, this can only happen under extreme conditions. To observe VHE $\gamma$-rays a few hundreds of hours after prompt emission, the required value of the Lorentz boost factor $(\Gamma)$ should be greater than 1000, whereas the expected value of $\Gamma$ is much less for afterglow emission. In this case, spectral energy distribution (from radio to $\gamma$-rays) can be fitted with a simple power-law model. On the other hand, for the SSC mechanism, the required electron energy is $\sim \mathrm{GeV}$ and that of $\Gamma$ is much less. VHE $\gamma$-rays can also be produced by hadronic processes e.g., proton synchrotron or via proton-proton or proton-photon interactions, however, these processes are less efficient compared to leptonic processes.

Ground-based $\gamma$-ray telescopes, particularly IACTs, are unlikely to detect GRBs during prompt emission, because of their small field of view, low duty cycle and also relatively higher energy threshold compared to the energy range in which prompt emission is expected. Air shower experiments are better suited in this regard as they have a wide field of view and they operate $24 \times 7$. On the other hand, these experiments have energy thresholds even higher than IACTs, about a few TeV or higher. There are only a handful of GRBs that are detected at GeV energies during prompt emission. Both IACTs and air shower experiments are better suited to detect afterglow emissions.

The H.E.S.S. telescope detected VHE emission from GRB 180702B, 10 hours after the prompt emission 223. This source was first identified by the GBM (Gamma-ray Burst Monitor) onboard Fermi $\gamma$-ray Space Telescope. The prompt emission lasted for 49 seconds, indicating that it was a long GRB. It was the 7th brightest among all GRBs detected by Fermi. An estimated redshift of this GRB, obtained from subsequent multi-wavelength follow-up of afterglow emission, is $z=0.653$. In two hours observations, total of $119 \gamma$-rays were detected in the energy range of 100-400 GeV by H.E.S.S. with a statistical significance of $5.3 \sigma$. Data were best fitted with a powerlaw spectrum. The power-law index was found to be consistent with the one fitted to the temporal decay flux observed in X-ray, optical afterglow emissions and with power-law index measured by Fermi-LAT (see Fig. 35). Such a broad single component is expected if VHE $\gamma$-rays are produced by synchrotron emissions by electrons. However, in order to produce $\gamma$-rays with energies around $100 \mathrm{GeV}$ and above via synchrotron emission, the required $\Gamma$ would be $>1000$, whereas a typical value of $\Gamma$ after 10 hours is expected to be around 20. On the other hand, SED can be explained 
using the SSC mechanism as this mechanism does not require extreme conditions to produce VHE $\gamma$-rays.

MAGIC telescope reported detection of one long burst GRB 190114C [224,225]. This GRB was first detected by Burst Alert Telescope (BAT) onboard the Neil Gehrels Swift Observatory. Multi-wavelength afterglow emission, which followed, revealed that this GRB was located at a redshift of 0.4245 . MAGIC started observing this GRB from 1 minute after Swift was triggered for 4.5 hours. VHE $\gamma$-rays were detected in the energy range $0.3-1 \mathrm{TeV}$. In the first 20 minutes, $\gamma$-rays were detected with very high significance $(\sim 50 \sigma)$. The combined light curve from $\mathrm{keV}$ to $\mathrm{TeV}$ have shown similar nature, which can be explained by a simple power-law spectrum. This points to the fact that during this time most of the emission is compatible with afterglow emission. However, it is possible that initially there was some overlap with prompt emission.

At lower energies up to a few $\mathrm{GeV}$, the emission is due to Synchrotron radiation by electrons. However, at higher energies contribution come from IC scattering of low energy synchrotron photons i.e. SSC mechanism (see Fig. 36).

H.E.S.S. reported detection of afterglow emission from yet another GRB, GRB 190829a 226]. This afterglow emission was observed for three nights. VHE photons were detected in the energy range of $0.18-3.3 \mathrm{TeV}$. Since the source is not very far $(\mathrm{z}=0.0785)$, a much smaller EBL correction was needed compared to previous GRBs. The intrinsic spectrum of the GRB measured by HESS smoothly matches with the X-ray spectrum. This indicates that both X-ray and $\gamma$-rays probably have the same origin, which means synchrotron emission is responsible for both the X-ray and VHE $\gamma$-ray emission. This raises questions about the current understanding of VHE emission mechanisms, i.e. $\gamma$-rays are produced by the SSC mechanism.

Apart from these GRBs, MAGIC has reported detection of VHE $\gamma$-ray emission from GRB 201216C. This is the farthest VHE $\gamma$-ray source detected so far, with a redshift of 1.1. The observations of this source began 57 seconds after the onset of the burst and $\gamma$-ray signal was detected at a significance level of $>5 \sigma$ [227]. Also, VHE detections with a significance level of $>3 \sigma$ have been reported by MAGIC from GRB 160821B [228] and GRB 201015A [229].

\subsection{Starburst Galaxies}

Starburst Galaxies (SBG) are the galaxies where massive stars are forming at a very high rate (about $10^{3}$ times greater than in a normal galaxy like our Milky Way). The optical and infrared (IR) luminosities observed from these sources are dominated by radiation from these young massive stars, indicating that there is a high concentration of gas and radiation in localized regions. These massive stars have relatively shorter lifetimes and at the end of their lifetime, they explode as supernovae. Thus starburst regions are an ideal environment for the acceleration of cosmic rays. These supernovae enrich the central star-forming regions with relativistic cosmic rays (protons, electrons and positrons). Cosmic ray energy densities in SBGs are orders of magnitude higher compared to normal galaxies. Cosmic ray protons produce pions by inelastic collisions with ambient gas particles (proton-proton interactions). HE and VHE $\gamma$-rays are produced from the decay of neutral pions, whereas the decay of charged pions produces neutrinos. Under a leptonic scenario, electrons can also produce $\gamma$-rays via bremsstrahlung or by upscattering low energy photons via IC scattering [230,231, 232. Till date two nearby SBGs NGC 253 (distance : $2500 \mathrm{kpc}$ ) and M82 (distance : $3900 \mathrm{kpc}$ ) are detected by ground-based IACTs. These are the weakest among various VHE sources detected. For NGC 253, starbursts activity is triggered by galaxy merger and for M82, it is due to interaction with nearby companion galaxy M81. 
The detection of NGC 253 was reported by H.E.S.S. collaboration in the year 2009 233. H.E.S.S. telescopes observed this source for a livetime of 119 hours during the years 2005, 2007 and 2008. The source was detected above $220 \mathrm{GeV}$ with a statistical significance of $5.2 \sigma$. The estimated integral flux was equivalent to $0.3 \%$ of the VHE $\gamma$-ray flux from the Crab Nebula. The H.E.S.S. collaboration again reported a joint analysis for this source along with Fermi-LAT data 234 in the year 2012. H.E.S.S. data with a duration of about 179 hours collected during 2005-2009 was analysed, whereas Fermi data spanned the period from 4th August 2008 to 3rd February 2011. H.E.S.S. detected NGC 253 with a significance level of $7.1 \sigma$. Both H.E.S.S. and Fermi spectra were best fitted with a power-law model. Later in 2018, using re-analysed H.E.S.S. data and Fermi-LAT data collected over a span of eight years, combined SED was generated, which is best described in terms of hadronic interactions [235], see Fig. 37

VERITAS collaboration reported detection of M82 in 2009 236] with a significance level of $4.8 \sigma$. The source was observed for a livetime of 137 hours during January 2008 - April 2009. The power-law index of the VHE $\gamma$-ray spectrum was found to be 2.5. Measured $\gamma$-ray flux above $700 \mathrm{GeV}$ was $0.9 \%$ of Crab nebula flux. Estimated cosmic ray density in the starburst region was $250 \mathrm{eV} \mathrm{cm}{ }^{-3}$, which is approximately 500 times the average cosmic ray density in our Milky Way. M82 was also detected by Fermi-LAT above $100 \mathrm{GeV}$ as reported in 237. Spectral data were fitted with a power-law with an index of 2.2. Like NGC 253, HE and VHE data of M82 have similar characteristics.

In both cases, the high density of cosmic rays in dense starburst region favours the hadronic origin of $\gamma$-ray emission. VHE $\gamma$-rays are produced by inelastic collisions between cosmic ray protons and target nuclei in the ambient ISM. Even though the data seem to favor the hadronic scenario, primary and secondary cosmic ray electrons can also contribute to VHE $\gamma$-rays via Bremsstrahlung and up-scattering of low-energy photons from ambient radiation fields by IC scattering.

\section{Fundamental Physics Aspects}

In this section, we review various fundamental physics aspects which can be studied exploring VHE $\gamma$-ray emission from various astrophysical sources.

\subsection{Extragalactic Background Light}

VHE $\gamma$-ray photons originating from distant sources like AGNs, GRBs etc interact with an extragalactic background light (EBL) on their way to the Earth. EBL is the diffuse radiation that has the second-highest energy density after the cosmic microwave background. It consists of the sum of the starlight emitted by galaxies through the history of the Universe. It is also important from a cosmology point of view as it has an important contribution from the first stars formed before the beginning of galaxy formation. SED of EBL consists of two bumps, the cosmic optical background and the cosmic infra-red background. The first bump corresponds to stellar emission from optical to NIR, whereas the second one corresponds to UVoptical light absorbed and re-radiated by dust in the IR domain (for a review, see [238]). Direct measurements of EBL are difficult due to foreground contamination due to zodiacal light etc and lead to overestimation. Strict lower limits are obtained from integrated galaxy counts $([239,240,241]$ etc $)$. Several models have been developed to describe this SED [242,243, 244, 245, 246, 247, 248, 249]. 
VHE $\gamma$-rays coming from astronomical sources interact with EBL photons producing electron-positron pairs. This leads to modification of the original spectrum. The observed flux is given by $F_{\text {obs }}=F_{\text {int }}(E) \times e^{-\tau(E)}$, where $F_{\text {int }}(E)$ is the intrinsic spectrum and $\tau(E)$ is the optical depth of EBL. Because of this interaction incident power-law spectrum gets modified with attenuation of flux depending on energy as well as distance travelled. This gives rise to the cosmic $\gamma$-ray horizon, the energydependent distance beyond which the optical depth due to this interaction exceeds unity. As a result, at lower redshifts, the Universe is more transparent and only the highest energy $\gamma$-rays are absorbed. Whereas, at higher redshifts, Universe is opaque to $\gamma$-rays of even lower energies. Interestingly, distortion of an incident spectrum caused by EBL can be used to get an estimate of EBL. The cosmic $\gamma$-ray horizon can also serve as a cosmological probe and also give an estimate of the distance to the source.

Amongst the present generation IACTs, first such attempt of getting EBL estimate from VHE $\gamma$-ray observations was done by H.E.S.S. collaboration using observations of two distant blazars detected that time, namely H 2356-309 ( $\mathrm{z}=0.165)$ and 1ES 1101$232(\mathrm{z}=0.186)$ 250. Using observed spectra of these sources with slopes of $2.88+/-0.17$ and $3.06+/-0.21$ respectively, measured over the energy ranges of $0.16-3.3$ and $0.16-1.1$ $\mathrm{TeV}$, and making a conservative estimate of intrinsic spectra to have indices of $\geq 1.5$ as expected in the scenario of shock acceleration of particles, and using plausible shape for SED of EBL, an upper limit on EBL was estimated for optical-NIR wavelengths. This limit was found to be very close to the lower limit given by the integrated light of resolved galaxies, indicating that intergalactic space is more transparent to $\gamma$-rays compared to what was thought previously. This work was extended using data on 1ES $0229+200(\mathrm{z}=0.1396)$ and a similar conclusion was arrived at [251].

Further extension of this work involved H.E.S.S. measurements of spectra of seven blazars with redshift in the range of 0.031-0.188, extending over the energy range of $100 \mathrm{GeV}$ to $5-10 \mathrm{TeV}$ 252. Carrying out the joint fit of the EBL optical depth and of the intrinsic spectra of the sources, assuming intrinsic smoothness, EBL flux density was constrained over $[0.3 \mu \mathrm{m}, 17 \mu \mathrm{m}]$ and the peak value at $1.4 \mu \mathrm{m}$ was derived as $\lambda f_{\lambda}=15 \pm 2$ (stat) \pm 3 (sys) $\mathrm{nW} \mathrm{m}^{-2} \mathrm{sr}^{-1}$. The EBL signature was detected at the level of $8.8 \sigma$. Fig. 38 shows EBL flux density as a function of wavelength obtained in this work.

More recent work from H.E.S.S. was based on the data from nine blazars covering a redshift range of 0.031-0.287 254. In this work, EBL signature was detected at a significance level of $9.5 \sigma$ and the intensity of EBL was estimated in four wavelength bands in the range of $0.25-98.6 \mu \mathrm{m}$ as shown in Fig. 39 .

A similar exercise was carried out by MAGIC collaboration using data from higher redshift object PG $1553+113(0.4<\mathrm{z}<0.58)$ [256] and from $1 \mathrm{ES} 1011+496(\mathrm{z}=0.212)$ during the flare state 257. More recent work used data on 12 blazars from FermiLAT and MAGIC and comparison of various EBL models was carried out 258. Estimates of the EBL density obtained were found to be in good agreement with models for EBL production and evolution. Using measurements of 14 VERITASdetected blazars, EBL SED was estimated in the wavelength range of $0.56-56 \mu \mathrm{m}$ and was found to be in good agreement with lower limits obtained by assuming that the EBL is entirely due to radiation from catalogued galaxies 259. Spectral measurements of the most distant blazars detected at VHE energies : FSRQ PKS $1441+25(\mathrm{z}=0.939)$ and gravitationally lensed blazar QSO B0218+357 $(\mathrm{z}=0.944)$ are also consistent with these conclusions [260,261]. 


\subsection{Lorentz Invariance Violation}

Several models of quantum gravity predict Lorentz symmetry to be broken at an energy scale around Planck scale $\left(E_{\text {Planck }}=\sqrt{h c^{5} / G} \approx 1.22 \times 10^{19} \mathrm{GeV}\right)$ (see e.g. $262,263,264)$. This is expected to produce energy-dependent dispersion and the modified dispersion relation is given as

$$
E^{2} \simeq p^{2} c^{2} \times\left[1 \pm\left(E / E_{Q G}\right)^{n}\right]
$$

where $c$ is the speed of light at the low-energy limit, $E$ and $p$ are energy and momentum of the photon, $E_{Q G}$ is the energy scale at which Lorentz invariance violation (LIV) would be seen, $n$ is the leading order of the LIV perturbation. The + sign corresponds to the superluminal case where photon speed decreases with an increase in energy whereas the - sign corresponds to the subluminal case with photon speed increasing with an increase in energy. $n=1$ corresponds to linear and $n=2$ corresponds to quadratic perturbation.

It is expected that comparison of arrival times of photons of different energies coming to the Earth from distant astronomical sources may show such dispersion 265 266. Short pulses of photons originating from distant sources with a large redshift spanning a wide energy range could be the best possibilities. Accordingly, AGNs, GRBs and pulsars were considered as the best candidates. It should be noted that arrival time delays between photons of different energies may partially arise due to other reasons e.g. from the emission mechanisms in the source itself, energy stratification induced at shock fronts or complex geometry of the emission zone. It is necessary to disentangle these effects to establish effects caused by LIV.

The first attempt of getting constraint on $E_{Q G}$ using VHE $\gamma$-ray data was done using observations of flare from Mrk 421 by the Whipple telescope [267. This flare was seen on 15 May 1996 with a doubling timescale of 15 minutes over $350 \mathrm{GeV}$ - $10 \mathrm{TeV}$. Using this data, limit on $E_{Q G}>6 \times 10^{16} \mathrm{GeV}$ was obtained. The first attempt using better quality data from present generation IACTs was based on the flare seen from Mrk 501 by MAGIC. Flux variation with doubling timescales down to 2 minutes was seen on two nights, 30 June 2005 and 9 July 2005 [156. The flare seen on 9 July with $\sim 20$ minutes duration showed an indication of a $4 \pm 1$ minute time delay between peaks of $<0.25 \mathrm{TeV}$ and $>1.2 \mathrm{TeV}$. This corresponds to the time delay of about $0.12 \mathrm{~s} / \mathrm{GeV}$. However, in the later work 268] it was noted that this approach is too simplistic. Considering the large width of the band, 1.2-10 TeV, compared to the energy difference between mean energies of the bands and noting a limited number of photons, the binned estimator was found to be inadequate for constraining linear and quadratic energy dependences. Instead, the method based on the time-energy distribution of individual photons was used and much lower values of delays were found. Based on these values, lower limit of $E_{Q G 1}>2.1 \times 10^{17} \mathrm{GeV}$ was derived for the linear case and $E_{Q G 2}>2.6 \times 10^{10} \mathrm{GeV}$ for the quadratic case at $95 \%$ confidence level 268. Using the refined method based on an unbinned likelihood estimator, limits of $E_{Q G 1}=0.3_{-0.10}^{+0.24} \times 10^{18} \mathrm{GeV}$ and $E_{Q G 2}=0.57_{-0.19}^{+0.75} \times 10^{11} \mathrm{GeV}$ were obtained for linear and quadratic cases respectively [269].

Another spectacular flare was seen by H.E.S.S. from blazar PKS 2155-304 on 28 July 2006 with the best-determined rise time of $173 \pm 28 \mathrm{~s}$ [157]. No significant energy-dependent time lag was seen in this flare and lower limits on the energy scale were derived [270. These limits were revised later using a more sensitive event-byevent method consisting of a likelihood fit. The previous limit on the linear term was improved by a factor of $\sim 3$ to $E_{Q G 1}>2.1 \times 10^{18} \mathrm{GeV}$. For quadratic term, sensitivity was lower and limit of $E_{Q G 2}>6.4 \times 10^{10} \mathrm{GeV}$ was obtained [271]. More recent data from H.E.S.S. telescope on blazars PG 1553+113 [186] and Mrk 501 [272] 
have also been used to derive limits on energy scales. Using MAGIC data on Crab pulsar at energies above $400 \mathrm{GeV}$ and carrying out the profile likelihood analysis, $95 \%$ confidence level limits were obtained on $E_{Q G 1}>5.5 \times 10^{17} \mathrm{GeV}$ (subluminal) and $4.5 \times 10^{17} \mathrm{GeV}$ (superluminal) for linear case and $E_{Q G 2}>5.9 \times 10^{10} \mathrm{GeV}$ (subluminal) and $5.3 \times 10^{10} \mathrm{GeV}$ (superluminal) for quadratic case [273]. Whereas in the most recent work involving MAGIC observations of GRB 190114C, limits of $E_{Q G 1}>5.8 \times 10^{18} \mathrm{GeV}$ (subluminal) and $5.5 \times 10^{18} \mathrm{GeV}$ (superluminal) were obtained for linear case and $E_{Q G 2}>6.3 \times 10^{10} \mathrm{GeV}$ (subluminal) and $5.6 \times 10^{10}$ $\mathrm{GeV}$ (superluminal) for quadratic case [274].

\subsection{Dark Matter}

One of the primary goals of VHE $\gamma$-ray astronomy is an indirect search for dark matter (DM). Based on the measurements of cosmic microwave background by the Planck satellite, about $85 \%$ of the matter in the universe is expected to be in the form of cold DM particles. The exact nature of DM is not known. It is likely to be made up of yet unknown elementary particles, which are expected to be massive $(\mathrm{GeV}$ $\mathrm{TeV}$ range), electrically neutral, stable (lifetime exceeding the age of the universe) and nonbaryonic. Possible candidates for cold DM are Weakly Interacting Massive Particles (WIMP). If DM consists of WIMP, it is expected to produce astrophysical $\gamma$-rays in various annihilation or decay processes either in the form of broad-band (sometimes with a sharp cutoff or bumps, depending on the DM model used) or line emission. In the last twenty years, various astrophysical sources have been studied to detect signatures of DM by H.E.S.S., MAGIC, VERITAS and Whipple telescopes. The number of observable $\gamma$-rays arising from DM annihilation depends on the annihilation cross-section as well as on the square of the number density of DM particles. Hence the astrophysical sources which are expected to have a high density of DM particles are the most promising targets. These targets include the Galactic Centre, galaxy clusters, dwarf spheroidal galaxies etc, which were prime targets for early observations carried out with present generation telescopes. Later other objects like intermediate black holes, DM subhalos were also explored along with the signatures for line emission.

One of the early observations from the present generation telescopes include observations of the Galactic Centre region by H.E.S.S. VHE $\gamma$-ray observations of the source HESS J1745-29, in the Galactic Centre region, in 2004, indicated the measured spectrum to be consistent with the power-law and a bulk of the VHE emission was found to have non-DM origin [275]. Further work based on observations of a region with projected distance $\mathrm{r} \sim 45-150 \mathrm{pc}$ from the Galactic Centre also did not show any hint of residual $\gamma$-ray flux after background subtraction 276. Using conventional profiles for DM density, limits were derived on the velocity-weighted annihilation cross-section $\langle\sigma v\rangle$ as a function of DM mass. For DM particle mass of $\sim 1 \mathrm{TeV}$, values of $\langle\sigma v\rangle$ above $3 \times 10^{-25} \mathrm{~cm}^{3} \mathrm{~s}^{-1}$ were excluded. Further work, based on ten years of observations carried out by H.E.S.S. resulted in limits of $6 \times 10^{-26} \mathrm{~cm}^{3}$ $\mathrm{s}^{-1}$ and $2 \times 10^{-26} \mathrm{~cm}^{3} \mathrm{~s}^{-1}$ for $\langle\sigma v\rangle$, respectively, for DM particle mass of $1 \mathrm{TeV}$ for $\mathrm{W}^{+} \mathrm{W}^{-}$channel and $1.5 \mathrm{TeV}$ for $\tau^{+} \tau^{-}$channel [277. Search for monoenergetic line emission produced by DM annihilation was also carried out using this data which revealed no significant $\gamma$-ray excess and limit of $4 \times 10^{-28} \mathrm{~cm}^{3} \mathrm{~s}^{-1}$ was obtained on $<\sigma v>$ at $1 \mathrm{TeV}[278$.

Another category of DM dominated objects extensively observed at VHE $\gamma$-ray energies is dwarf spheroidal galaxies (dSphs). These are the satellite galaxies gravitationally bound to the Milky Way, located in the Milky Way DM halo. These are particularly interesting targets with a high mass-to-light ratio. As they have relatively low star content, minimizing the contribution to $\gamma$-ray production from usual 
astrophysical sources like supernova activity, they provide a better opportunity to detect DM. The dSphs observed by H.E.S.S., MAGIC and VERITAS include Sagittarius, Canis Major, Sculptor, Carina, Coma Berenices, Fornax, Dark Energy Survey (DES) dwarf galaxies, Draco, Ursa Minor, Boötes 1, Willman 1, Segue 1, Ursa Major II, Triangulum II, dwarf irregular galaxy WLM etc (see for example, 279,280, 281, 282, 283, 284, 285, 286, 287, 288, 289, 290, 291, 292, 293]) Limits on $\langle\sigma v>$ obtained from these observations span the range of $\sim 10^{-22}$ to $\sim 10^{-26} \mathrm{~cm}^{3} \mathrm{~s}^{-1}$ for DM particle mass range of about few 100's GeV to few 10's TeV. As an example, in Fig. 40, 95\% confidence level upper limits on $\langle\sigma v\rangle$ are shown over the DM mass range of $10 \mathrm{GeV}$ - $100 \mathrm{TeV}$ obtained by combining observations of Segue 1 from MAGIC and of 15 dSphs from Fermi-LAT [289]. Four panels of the figure correspond to the annihilation of DM particles into standard model pairs $b \bar{b}, W^{+} W^{-}, \tau^{+} \tau^{-}$and $\mu^{+} \mu^{-}$. It demonstrates nicely how the combination of Fermi-LAT observations with those from ground-based IACTs can lead to estimation of DM annihilation cross-section limits over a wide mass range.

Next class of sources widely observed in search for DM signatures is galaxy clusters. Observations of Fornax 294, Coma 295 and Perseus 296, 297 clusters have resulted in the limits on $\langle\sigma v\rangle$ in the range of $\sim 10^{-22}$ to $\sim 10^{-26} \mathrm{~cm}^{3} \mathrm{~s}^{-1}$. From observations of globular clusters like NGC 6388 and M15 298, 291] and local group galaxies M32, M33 291 similar upper limits were obtained. Another interesting class of objects is intermediate mass black holes (IMBHs) with mass in the range of $10^{2}-10^{6}$ $\mathrm{M}_{\odot}$. IMBHs are expected to be DM annihilation boosters giving rise to 'mini-spikes' around their locations. H.E.S.S. data from inner Galactic plane survey was examined for such signatures and scenario for DM particle annihilation was excluded at $90 \%$ confidence level with $\langle\sigma v\rangle$ above $10^{-28} \mathrm{~cm}^{3} \mathrm{~s}^{-1}$ for DM mass between $800 \mathrm{GeV}$ and $10 \mathrm{TeV}$ [299].

\section{Conclusions}

The last two decades have witnessed a spectacular journey for VHE ground-based $\gamma$-ray astronomy. From a handful of sources at the beginning of the century, the $\mathrm{TeV}$ catalog has now reached around 250 galactic and extra-galactic sources. Our understanding of the VHE $\gamma$-ray universe has made giant strides with new discoveries and observations (extreme conditions prevailing in jets of AGNs, surroundings of GRBs, possible signatures of PeVatrones, pulsed emission etc) coupled with better theoretical and phenomenological models to explain these fascinating observations. However, the journey is far from reaching saturation and the latest results have raised many more questions which need deeper probes of the already discovered systems, multiple and long duration surveys and many more simultaneous multi-wavelength campaigns between different observatories across the electromagnetic spectrum. At the same time, several outstanding problems like a definite proof of acceleration of hadrons to $\mathrm{PeV}$ energies and identification of dark matter which is supposed to pair annihilate to high energy gamma rays remain elusive. Once the next generation of telescopes like CTA, SWGO and the completely installed LHAASO start to take data, our understanding of the very high energy Universe will undergo a quantum jump. With much improved sensitivity and angular resolution, CTA is expected to detect many more sources. Besides revealing the nature of astrophysical sources under relativistic conditions, these $\gamma$-rays will hopefully also probe fundamental physics problems such as measuring the energy spectra of the extra-galactic background light (EBL), probing quantum gravity effect and searching for dark matter. The future of VHE $\gamma-$ ray astronomy indeed looks extremely bright. 


\section{Acknowledgement}

DB acknowledges Science and Engineering Research Board - Department of Science and Technology for Ramanujan Fellowship - SB/S2/ RJN-038/2017. PM acknowledges the generous support of the Stanislaw Ulam fellowship by Polish National Agency for Academic Exchange (NAWA). VRC acknowledges the support of the Department of Atomic Energy, Government of India, under Project Identification No. RTI4002.

\section{References}

1. S. P. Swordy, Space Science Reviews 99, (2001) 85

2. E. Fermi, Physical Review 75, (1949) 1169

3. E. Fermi, The Astrophysical Journal 119, (1954) 1

4. M. S. Longair, High Energy Astrophysics Cambridge University Press, 1981

5. G. R. Blumenthal \& R. J. Gould, Reviews of Modern Physics 42, (1970) 237

6. T. C. Weekes et al., The Astrophysical Journal 342, (1989) 379

7. S. LeBohec et al., The Astrophysical Journal 539, (2000) 209

8. F. Aharonian et al., Astronomy \& Astrophysics, 375 (2001), 1008

9. F. Aharonian et al., Astronomy \& Astrophysics, 395 (2002), 3

10. F. Aharonian et al., Science, 307 (2005), 1938

11. F. Aharonian et al., The Astrophysical Journal, 636 (2006), 777

12. H. Abdalla et al., Astronomy \& Astrophysics, 612 (2018), A1

13. R. Adam, P. A. R. Ade et al., Astronomy \& Astrophysics, 594 (2016), A10

14. A. Weinstein et al. 2009, arXiv: 0912.4492

15. A. U. Abeysekara et al., The Astrophysical Journal, 861 (2018), 134

16. A. Albert et al., The Astrophysical Journal, 905 (2020), 76

17. D. Ellison et al, The Astrophysical Journal, 487 (1997), 197

18. L. Drury, Reports on Progress in Physics, 46 (1983), 973

19. R. Blandford and D. Eichler, Physics Reports, 154 (1987), 1-75

20. R. Blandford and J. P. Ostriker, The Astrophysical Journal, 221 (1978), L29

21. C. Jones, The Astrophysical Journal Supplement Series, 90 (1994), 561

22. A. Malkov and L. O'C Drury, Reports on Progress in Physics, 64 (2001), 429

23. S. Funk, Annual Review of Nuclear and Particle Science, 65 (2015), 245

24. F. Aharonian et al, Nature, 432 (2004), 75-77

25. F. Aharonian et al, Astronomy \& Astrophysics, 437 (2005), L7-L10

26. F. Acero et al, Astronomy \& Astrophysics, 516 (2010), A62

27. A. Abramowski et al, Astronomy \& Astrophysics, 531 (2011), A81

28. F. Aharonian et al, The Astrophysical Journal, 692 (2009), 1500-1505

29. J. Albert et al, Astronomy \& Astrophysics, 474 (2007), 937-940

30. V. A. Acciari et al, The Astrophysical Journal, 714 (2010), 163

31. M. L. Ahnen et al, Monthly Notices of the Royal Astronomical Society, 472 (2017), 2956

32. S. Archambault et al, The Astrophysical Journal, 836 (2017), 23

33. J. Albert et al., The Astrophysical Journal, 664 (2007), L87-L90

34. V.A. Acciari et al., The Astrophysical Journal, 698 (2009), L133-L137

35. A. U. Abeysekara et al, The Astrophysical Journal, 894 (2020), 13

36. M. Ackermann et al, Science, 339 (2013), 807

37. L. Saha et al, Astronomy \& Astrophysics, 563 (2014), A88

38. H. Muraishi et al, Astronomy \& Astrophysics, 354 (2000), L57-61

39. D. C Ellison et al, The Astrophysical Journal, 712 (2010), 287

40. T. Tanaka et al, The Astrophysical Journal, 685, (2008), 988

41. F. Aharonian et al, Astronomy \& Astrophysics, 449 (2006), 223

42. F. Aharonian et al, Astronomy \& Astrophysics, 464 (2007), 235

43. A. Abdo et al, The Astrophysical Journal, 734 (2011), 9

44. H. Abdalla et al, Astronomy \& Astrophysics, 612 (2018), A5 
45. E. Aliu et al, The Astrophysical Journal, 770 (2013), 93

46. V. Acciari et al, to be published in Astronomy \& Astrophysics, arXiv:2010.15854

47. N. Fraija and Araya, M, The Astrophysical Journal, 826 (2016), 8

48. M. Araya and Fraija, N, AIP Conference Proceedings, 1792 (2017), 1

49. A. A. Abdo, et al, Science, 325 (2009), 840

50. A. A. Abdo et al, The Astrophysical Journal, 712 (2010), 459-468

51. M. Lemoine-Goumard, Proceedings of Science, The 34th International Cosmic Ray Conference, 012,2015

52. A. Abramowski et. al., Astronomy \& Astrophysics, 612 (2018), A4

53. H. Abdalla et. al., Astronomy \& Astrophysics, 612 (2018), A5

54. H. Abdalla et. al., Astronomy \& Astrophysics, 612 (2018), A7

55. H. Abdalla et. al., Astronomy \& Astrophysics, 644 (2020), A112

56. A. U. Abeysekara et. al., Physical review letters, 124, 021102, 2020

57. B. M. Gaensler and P. O. Slane, Astronomy \& Astrophysics, 44 (2006), 17-47

58. F. A. Aharonian, S. V. Bogovalov \& D. Khangulyan, Nature 482 (2012), 507-509

59. A. M. Atoyan \& F. A. Aharonian, MNRAS, 278, (1996), 525

60. A. A. Abdo et al., The Astrophysical Journal, 708 (2010), 1254

61. V. A. Acciari et al, Astronomy \& Astrophysics, 635 (2020), A158

62. M. Amenomori et al, Physical review Letters, 123 (2019), 051101

63. A. U. Abeysekara et al, The Astrophysical Journal, 881 (2019), 13

64. Zhen Cao et. al., Nature, 594 (2021), 33-36

65. H. Abdalla et al, Astronomy \& Astrophysics, 612 (2018), A2

66. F. Aharonian et al, Astronomy \& Astrophysics, 460 (2006), 365-374

67. H. Abdalla et. al., Astronomy \& Astrophysics, 621 (2019), A116

68. H. Abdalla et. al., Astronomy \& Astrophysics, 627 (2019), A100

69. A. A. Abdo et al, The Astrophysical Journal, 664 (2007), L91-94

70. A. U. Abeysekara et al, The Astrophysical Journal, 861 (2018), 33

71. E. Aliu et al, The Astrophysical Journal, 788 (2014), 10

72. F. Brun et al, Proceedings of the 25th Texas Symposium on Relativistic Astrophysics, 201, 2010

73. Abraham Luna et al, The Astrophysical Journal Letters, 713 (2010), L45-49

74. I. F. Mirabel, Science, 335 (2012), 175

75. F. Aharonian et al. Science, 309 (2005), 746-749

76. J. M. Paredes et al., Science, 288 (2000), 5475

77. F. Aharonian et al., Astronomy \& Astrophysics, 460 (2006), 743

78. J. Casares et al., Monthly Notices of the Royal Astronomical Society, 364 (2005), 899

79. J. Albert et al., Science, 312 (2006), 1771

80. J. B. Hutchings, D. Crampton, Pub. Astron. Soc. Pacific 93, 486 (1981)

81. P. C. Gregory, The Astrophysical Journal, 575 (2002), 427

82. H. Anderhub et al., The Astrophysical Journal Letters, 706 (2009), L27

83. V. A. Acciari et al., The Astrophysical Journal, 738 (2011), 3

84. E. Aliu et al., The Astrophysical Journal, 779 (2013), 88

85. S. Johnston et al., Monthly Notices of the Royal Astronomical Society, 255 (1992), 401

86. S. Johnston et al., The Astrophysical Journal Letters, 387 (1992), L37

87. F. Aharonian et al., Astronomy \& Astrophysics, 442 (2005), 1-10

88. S. Johnston et al., Monthly Notices of the Royal Astronomical Society, 358 (2005), 1069

89. F. Aharonian et al., Astronomy \& Astrophysics, 507 (2009), 389-396

90. A. Abramowski et al., Astronomy \& Astrophysics, 551 (2013), A94

91. H. Abdalla et al., Astronomy \& Astrophysics, 633 (2020), A102

92. A.U. Abeysekara et al., The Astrophysical Journal Letters, 867 (2018), L19

93. H. Abdalla et al., Astronomy \& Astrophysics, 610 (2018), L17

94. H. Abdalla et al., Astronomy \& Astrophysics, 635 (2020), A167

95. A.U. Abeysekara et al., Nature, 562 (2018), 82

96. M. A. Ruderman and P. G. Sutherland, The Astrophysical Journal, 196 (1975), 51

97. J. K. Daugherty and A. K. Harding, The Astrophysical Journal, 252 (1982), 337

98. M. G. Baring, Advances in Space Research, 33 (2004), 552 
99. J. Arons and E. T. Scharlemann, The Astrophysical Journal, 231 (1979), 854

100. A. G. Muslimov and A. K. Harding, The Astrophysical Journal, 606 (2004), 1143

101. A. K. Harding et al., The Astrophysical Journal, 680 (2008), 1378

102. K. S. Cheng et al., The Astrophysical Journal, 300 (1986), 500

103. K. Hirotani, arXiv :0809.1283, (2008).

104. A. P. S. Tang et al., The Astrophysical Journal, 676 (2008), 562

105. E. Aliu et al., Science, 322 (2008), 1221

106. J. Albert et al. The Astrophysical Journal, 674 (2008), 1037

107. E. Aliu et al., Science, 334 (2011), 69

108. A. Abdo et al., The Astrophysical Journal, 708 (2010), 1254

109. J. Aleksić et al., The Astrophysical Journal, 742 (2011), 43

110. J. Aleksić et al., Astronomy \& Astrophysics, 540 (2012), A69

111. J. Aleksić et al., Astronomy \& Astrophysics, 565 (2014), L12

112. S. Ansoldi et al., Astronomy \& Astrophysics, 585 (2016), A133

113. H. Abdalla et al., Astronomy \& Astrophysics, 620 (2018), A66

114. V. A. Acciari et al., Astronomy \& Astrophysics, 643 (2020), L14

115. R. Aloisio et. al., Astroparticle Physics (Springer), 2018

116. M. Amenomori et. al.,Nature Astronomy, 5 (2021), 460-464

117. Zhen Cao et. al., Science, 373 (2021), 425-430

118. Albert, A. et al., The Astrophysical Journal, 896 (2020), L29-L37

119. V. A. Acciari et al., The Astrophysical Journal, 703 (2009), L6-L9

120. A. Abramowski et. al., Nature, 531 (2016), 476-479

121. R. D. Blandford \& R. L. Znajek, Monthly Notices of the Royal Astronomical Society, 179 (1977), 433

122. R. D. Blandford \& D. G. Payne, Monthly Notices of the Royal Astronomical Society, 199 (1982), 199

123. R. Antonucci, Annual Review of Astronomy and Astrophysics, 31 (1993), 473

124. P. Urry \& P. Padovani, Publications of the Astronomical Society of the Pacific, 107 (1995), 803

125. M. Sikora, M. C. Begelman and M. J. Rees, The Astrophysical Journal, 421 (1994), 153

126. F. A. Aharonian, New Astronomy, 5 (2000), 377

127. A. Mücke et al., Astroparticle Physics, 18 (2003), 593

128. K.Mannheim, Biermann P. L., Astronomy \& Astrophysics, 221 (1989), 211

129. K. Mannheim, Science, 279 (1998), 684

130. IceCube Collaboration, Science, 361 (2018), 147

131. G. Fossati et al., Monthly Notices of the Royal Astronomical Society, 299 (1998), 433

132. D. Donato et al., Astronomy \& Astrophysics, 375 (2001), 739

133. M. Punch et al., Nature, 358 (1992), 477

134. J. Quinn et al., The Astrophysical Journal Letters, 456 (1996), L83

135. V. A. Acciari et al., The Astrophysical Journal Letters, 684 (2008), L73

136. J. Albert et al., Science, 320 (2008), 1752

137. M. Tluczykont et al., Astronomy \& Astrophysics, 524 (2010), A48

138. F. Aharonian et al., Astronomy \& Astrophysics, 327 (1997), L5

139. H. Krawczynski et al., The Astrophysical Journal, 601 (2004), 151

140. V. A. Acciari et al., Astronomy \& Astrophysics, 638 (2020), A14

141. E. Aliu et al., The Astrophysical Journal, 750 (2012) 94

142. A. Abramowski et al., Astronomy \& Astrophysics, 538 (2012) A103

143. A. Abramowaki et al., Monthly Notices of the Royal Astronomical Society, 434 (2013) 1889

144. A. U. Abeysekara et al., The Astrophysical Journal, 836 (2017) 205

145. M. L. Ahnen et al., Astronomy \& Astrophysics 603 (2017) A29

146. V. A. Aleksić et al., The Astrophysical Journal, 748 (2012) 46

147. A. A. Abdo et al., The Astrophysical Journal, 736 (2011), 131

148. J. Aleksić et al., Astronomy \& Astrophysics, 576 (2015), 126

149. V. A. Acciari et al., The Astrophysical Journal Supplement, 248 (2020), 29 
150. J. Quinn et al., The Astrophysical Journal, 518 (1999), 693

151. M. L. Ahnen et al., Astronomy \& Astrophysics, 603 (2017), A31

152. M. L. Ahnen et al., Astronomy \& Astrophysics, 620 (2018), A181

153. H. Abdalla et al. Astronomy \& Astrophysics 648 (2021), A23

154. T. Arlen et al., The Astrophysical Journal, 762 (2012), 92

155. J. Aleksić et al., Astronomy \& Astrophysics, 542 (2012), A100

156. J. Albert et al., The Astrophysical Journal 669 (2007), 862

157. F. Aharonian et al., The Astrophysical Journal Letters, 664(2007) L71

158. A. U. Abeysekara et al., The Astrophysical Journal, 890 (2020), 97

159. M. L. Ahnen et al., The Astrophysical Journal Letters, 815 (2015) L23

160. J. Aleksić et al., The Astrophysical Journal Letters, 730 (2011), L8

161. H. T. Liu and J. M. Bai, The Astrophysical Journal, 653 (2006), 1089

162. E. Lefa et al., The Astrophysical Journal Letters, 743 (2011), L19

163. M. C. Begelman et al., Monthly Notices of the Royal Astronomical Society Letters, 384 (2008), L19-L23

164. A. Shukla, K. Mannheim, NatCo, 11 (2020), 4176

165. P. Subramanian et al. Monthly Notices of the Royal Astronomical Society 423 (2012) 1707

166. M. Blazejowski et al., The Astrophysical Journal, 630 (2005) 130

167. J. Albert et al., The Astrophysical Journal, 663 (2007), 125-138

168. V. Acciari et al., Astroparticle Physics, 54 (2014), 1

169. J. Aleksić et al Astronomy \& Astrophysics, 573 (2015), 50

170. A. Furniss et al., The Astrophysical Journal, 812 (2015), 65

171. V. A. Acciari et al., The Astrophysical Journal, 738 (2011), 169

172. F. Aharonian et al., Astronomy \& Astrophysics, 502 (2009), 749

173. V. A. Acciari et al., The Astrophysical Journal, 703 (2009), 169-178

174. F.Aharonian et al., The Astrophysical Journal Letters, 696 (2009), L150

175. S. Vaughan et al., Monthly Notices of the Royal Astronomical Society, 345 (2003), 1271

176. V. A. Acciari et al., Astronomy \& Astrophysics, 637 (2020), 86

177. M. L. Ahnen et al., Astronomy \& Astrophysics, 593 (2019), A91

178. A. U. Abeysekara et al., The Astrophysical Journal, 834 (2017), 2

179. J. Aleksic et al., Astronomy \& Astrophysics, 572 (2014), A121

180. A. Sinha et al, Astronomy \& Astrophysics, 591 (2016), A83

181. M. Baloković et al., The Astrophysical Journal, 819 (2016), 156

182. V. A. Acciari et al., Monthly Notices of the Royal Astronomical Society, bf 504 (2021), 1427

183. H. Abdalla et al., Astronomy \& Astrophysics, 598 (2017), A39

184. V. A. Acciari et al., The Astrophysical Journal, 729 (2011), 2

185. J. Albert et al, The Astrophysical Journal, 639 (2006), 761

186. A. Abramowski et al., The Astrophysical Journal, 802 (2015), 65

187. V. A. Acciari et al., The Astrophysical Journal Letters, 693 (2009), L104

188. I. Donnarumma et al., The Astrophysical Journal Letters, 691 (2009), L13

189. A. Shukla et al., Astronomy \& Astrophysics, 541 (2012), A140

190. E. Pian et al., The Astrophysical Journal Letters, 492 (1998), L17

191. G. Tagliaferri et al., The Astrophysical Journal, 679 (2008), 1029

192. E. Aliu et al., The Astrophysical Journal, i797 (2014), 89

193. J. Albert et al., The Astrophysical Journal, 662 (2007), 892

194. V. A. Acciari et al., Monthly Notices of the Royal Astronomical Society, 496 (2020), 3912

195. J. Valverde et al., The Astrophysical Journal, 891 (2020), 170

196. A. Shukla et al., The Astrophysical Journal, 798 (2015), 2

197. J. Aleksić et al., Astronomy \& Astrophysics, 578 (2015), A22

198. M. Sikora et al., The Astrophysical Journal, 577 (2002), 78

199. H. Abdalla et al., Astronomy \& Astrophysics, 627 (2019), A159

200. J. Aleksić et al., Astronomy \& Astrophysics 569 (2014), 46 
201. V. A. Acciari et al., Astronomy \& Astrophysics, 619 (2018), A159

202. M. Ackermann1 et al., The Astrophysical Journal, 786 (2014), 157

203. V. A. Acciari et al., The Astrophysical Journal, 707 (2009), 612

204. J. Aleksić et al., Astronomy \& Astrophysics, 530 (2011), A4

205. S. Archambault et al., Monthly Notices of the Royal Astronomical Society, 461 (2016), 202

206. E. Aliu et al., The Astrophysical Journal, 742 (2011), 127

207. E. Aliu et al., The Astrophysical Journal, 755 (2012), 118

208. IceCube Collaboration et al., Science, 361 (2018), eaat1378

209. IceCube Collaboration et al., Science, 361 (2018), 147-151

210. Kadler et al., NatPh, 12 (2016,) 807

211. J. Aleksić et al., Science, 346 (2014) 1080

212. H. Abdalla et al, Nature, 582 (2020) 356

213. Aharonian et al. Science, 314 (2006) 1424

214. A. Archer et al, The Astrophysical Journal, 896 (2020)

215. A. Abramowski et al., The Astrophysical Journal, 746 (2011) 151

216. V. A. Acciari et al., Science,325 (2009) 444

217. N. Gehrels, \& P. Mészáros, Science, 337 (2012), 932-936

218. P. Kumar, P. \& B. Zhang, Physics Reports, 561 (2015), 1-109

219. P. Mészáros et. al., New Astronomy Review, 48 (2004), 445-451

220. Y.-Z Fan \& T. Piran, Front. Phys. China, 3 (2008), 306-330

221. S. Inoue et al., Astroparticle Physics, 43 (2013), 252-275

222. B. Zhang, Nature, 575 (2019), 448-449

223. H. Abdalla et al., Nature, 575 (2019), 464-467

224. V. A. Acciari et al., Nature, 575 (2019), 455-458

225. V. A. Acciari et al., Nature, 575 (2019), 459-463

226. H. Abdalla et al., Science, 372 (2021), 1081-1085

227. O. Blanch et al., The Astronomer's Telegram, 14275 (2020) 1

228. V. A. Acciari et al., The Astrophysical Journal, 908 (2021), 90

229. O. Blanch et al., The $\gamma$-ray Coordinates Network, 28659 (2020) 1

230. H. J. Völk et. al., Space Science Review, 75 (1996), 279

231. T. A. D. Paglione et. al., The Astrophysical Journal, 460 (1996), 295

232. F. A. Aharonian et. al., Nature, 432 (2004), 75

233. F. Acero et. al., Science, 326 (2009), 1080-1082

234. A. Abramowski et. al., The Astrophysical Journal, 757 (2012), 158

235. H. Abdalla et al., Astronomy \& Astrophysics, 617 (2018), A73

236. V. A. Acciari et. al., Nature, 462 (2009), 770-772

237. A. A. Abdo et. al., The Astrophysical Journal, 709 (2010), L152

238. M. G. Hauser and E. Drek, Annual Review of Astronomy and Astrophysics, 39 (2001), 249

239. P. Madau and L. Pozzetti, Monthly Notices of the Royal Astronomical Society, 312 (2000), L9

240. G. G. Fazio et al., The Astrophysical Journal Supplement Series, 154 (2004), 39

241. H. Dole et al., Astronomy \& Astrophysics, 451 (2006), 417

242. A. Franceschini et al., Astronomy \& Astrophysics, 487 (2008), 837

243. A. Dominguez et al., Monthly Notices of the Royal Astronomical Society, 410 (2011), 2556

244. T. M. Kneiske and H. Dole, Astronomy \& Astrophysics, 515 (2010), A19

245. J. D. Fincke et al., The Astrophysical Journal, 712 (2010), 238

246. R. C. Gilmore et al., Monthly Notices of the Royal Astronomical Society, 422 (2012), 3189

247. K. Helgason and A. Kashlinsky, The Astrophysical Journal, 758 (2012), L13

248. Y. Inoue et al., The Astrophysical Journal, 768 (2013), 197

249. F. W. Stecker et al., The Astrophysical Journal, 827 (2016), 6

250. F. Aharonian et al., Nature, 440 (2006), 1018

251. F. Aharonian et al., Astronomy \& Astrophysics, 475 (2007), L9 
252. A. Abramowski et al., Astronomy \& Astrophysics, 550 (2013), A4

253. M. Meyer et al., Astronomy \& Astrophysics, 542 (2012), A59

254. H. Abdalla et al., Astronomy \& Astrophysics, 606 (2017), A59

255. J. Biteau and D. A. Williams, The Astrophysical Journal, 812 (2015), 60

256. J. Aleksic et al., Monthly Notices of the Royal Astronomical Society, 450 (2015), 4399

257. M. L. Ahnen et al., Astronomy \& Astrophysics, 590 (2016), 24

258. V. A. Acciari et al., Monthly Notices of the Royal Astronomical Society, 486 (2019), 4233

259. A. U. Abeysekara et al., The Astrophysical Journal, 885 (2019), 150

260. A. Abeysekara et al., The Astrophysical Journal Letters, 815 (2015), L22

261. M. L. Ahnen et al., Astronomy \& Astrophysics, 595 (2016), A98

262. T. Jacobson et al. Annals of Physics, 321 (2006), 150

263. G. Amelino-Camelia, Living Reviews in Relativity, 16 (2013), 5

264. N. E. Mavromatos, International Journal of Modern Physics A, 25 (2010), 5409

265. G. Amelino-Cameliai et al., Nature, 393 (1998), 763

266. J. R. Ellis et al., Nature, 428 (2004), 386

267. S. D. Biller et al., Physical Review Letters, 83 (1999), 2108

268. J. Albert, et al., Physics Letters B, 668 (2008), 253

269. M. Martinez and M. Errando, Astroparticle Physics, 31 (2009), 226

270. F. Aharonian et al., Physical Review Letters, 101 (2008), 170402

271. A. Abramowski et al., Astroparticle Physics, 34 (2011), 738

272. H. Abdalla et al., The Astrophysical Journal, 870 (2019), 93

273. M. L. Ahnen et al., The Astrophysical Journal Supplement Series, 232 (2017), 9

274. V. A. Acciari et al., Physical Review Letters, 125 (2020), 021301

275. F. Aharonian et al., Physical Review Letters, 97 (2006), 221102

276. A. Abramowski et al., Physical Review Letters, 106 (2011), 161301

277. H. Abdallah et al., Physical Review Letters, 117 (2016), 151302

278. H. Abdallah et al., Physical Review Letters, 120 (2018), 201101

279. F. Aharonian et al., Astroparticle Physics, 29 (2008), 55

280. F. Aharonian et al., The Astrophysical Journal, 691 (2009), 175

281. A. Abramowski et al., Astroparticle Physics, 34 (2011), 608

282. A. Abramowski et al., Physical Review D, 90 (2014), 112012

283. M. Cirelli et al., Journal of Cosmology and Astroparticle Physics, 11 (2018), 037

284. H. Abdallah et al., Physical Review D, 102 (2020), 062001

285. H. Abdallah et al., Physical Review D, 103 (2021), 102002

286. V. A. Acciari et al., The Astrophysical Journal, 720 (2010), 1174

287. E. Aliu et al., Physical Review D, 85 (2012), 062011

288. J. Aleksić et al., Journal of Cosmology and Astroparticle Physics, 06 (2011), 035

289. J. Aleksić et al., Journal of Cosmology and Astroparticle Physics, 02 (2016), 039

290. S. Archambault et al., Physical Review D, 95 (2017), 082001

291. M. Wood et al., The Astrophysical Journal, 678 (2008), 594

292. M. L. Ahnen et al., Journal of Cosmology and Astroparticle Physics, 03 (2018), 009

293. V. A. Acciari et al., Physics of the Dark Universe, 28 (2020), 100529

294. A. Abramowski et al., The Astrophysical Journal, 750 (2012), 123

295. T. Arlen et al., The Astrophysical Journal, 757 (2012), 123

296. J. Aleksić et al., The Astrophysical Journal, 710 (2010), 634

297. V. A. Acciari et al., Physics of the Dark Universe, 22 (2018), 38

298. A. Abramowski et al., The Astrophysical Journal, 735 (2011), 12

299. F. Aharonian et al., Physical Review D, 78 (2008), 072008

300. B. S. Acharya et al, Astroparticle Physics, 43 (2013), 3

301. Science with CTA, by CTA Consortium, World Scientific Press, 2019 


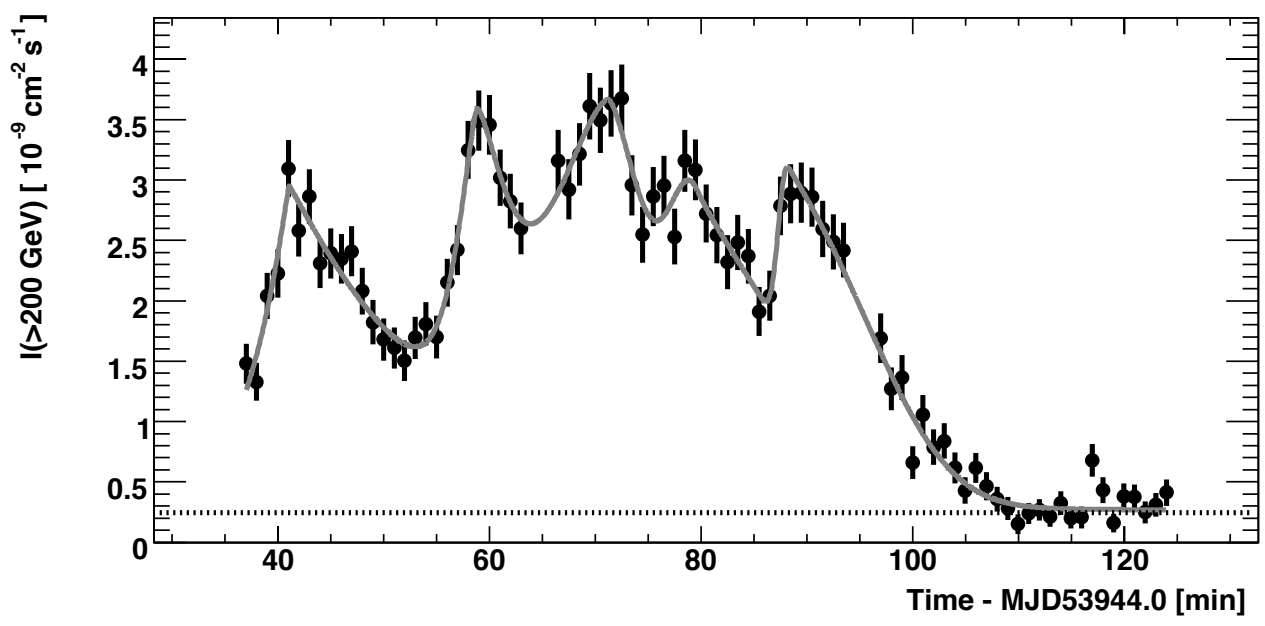

Fig. 23. Spectacular flare with rapid variability from PKS 2155-304 as observed by HESS. Adapted from [157] with permission. 


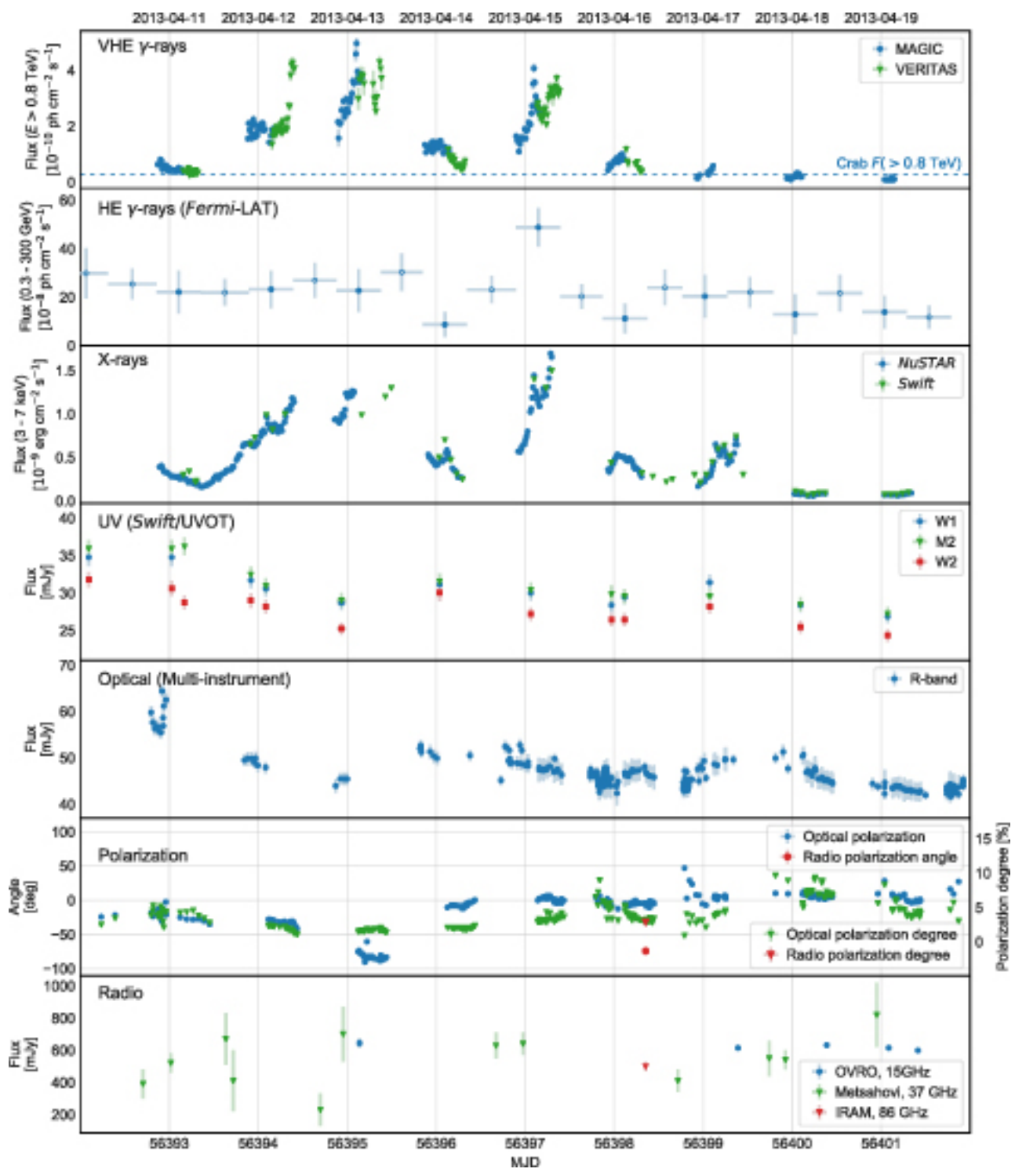

Fig. 24. Multiwaveband light curve of Mrk 421 during flaring activity in April 2013, covering data from radio, optical, UV, X-ray HE and VHE $\gamma$-ray bands. Adapted from [149] with permission. 


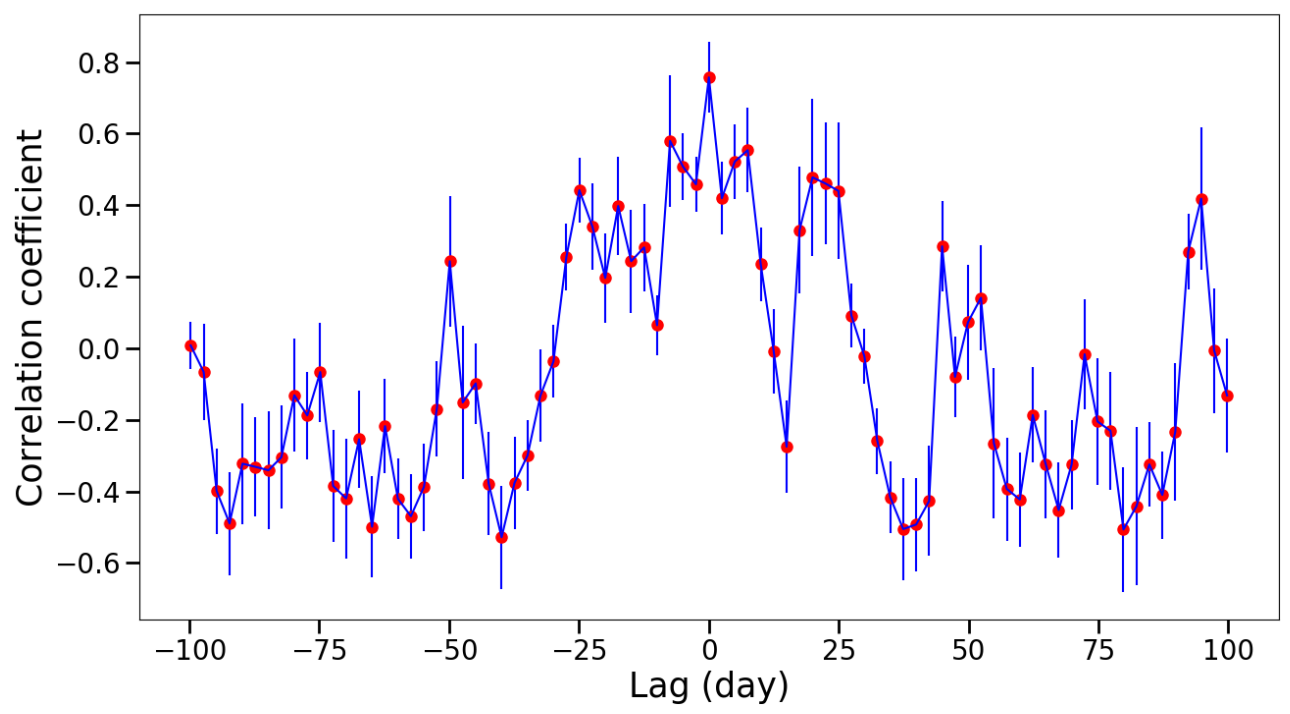

Fig. 25. Discrete correlation function as a function of time lag for VHE $\gamma$-ray and X-ray light curves of 1ES1959+650 over the lag of -100 to +100 days. VHE flux shows correlation with X-ray flux with DCF $\sim 0.76 \pm 0.1$ with zero time lag. Adapted from [140] with permission.

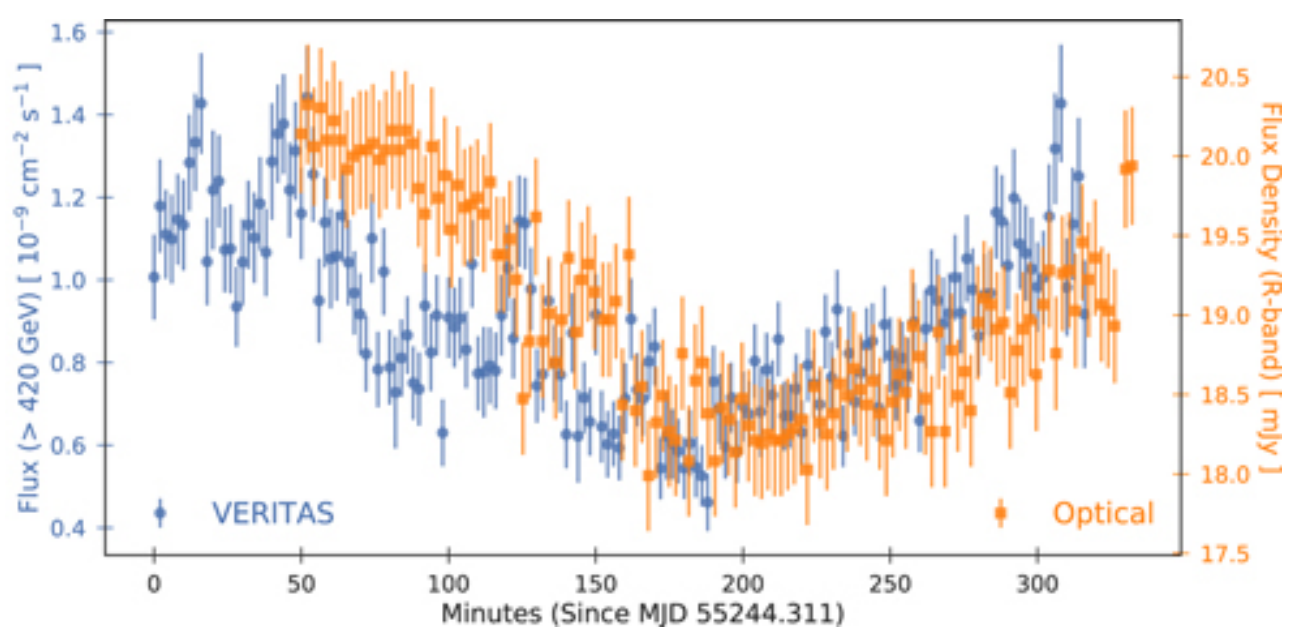

Fig. 26. The 2-minute binned VHE light curve $>420 \mathrm{GeV}$ from VERITAS (blue) and optical R band light curve from RCT (orange). Adapted from 158 with permission. 


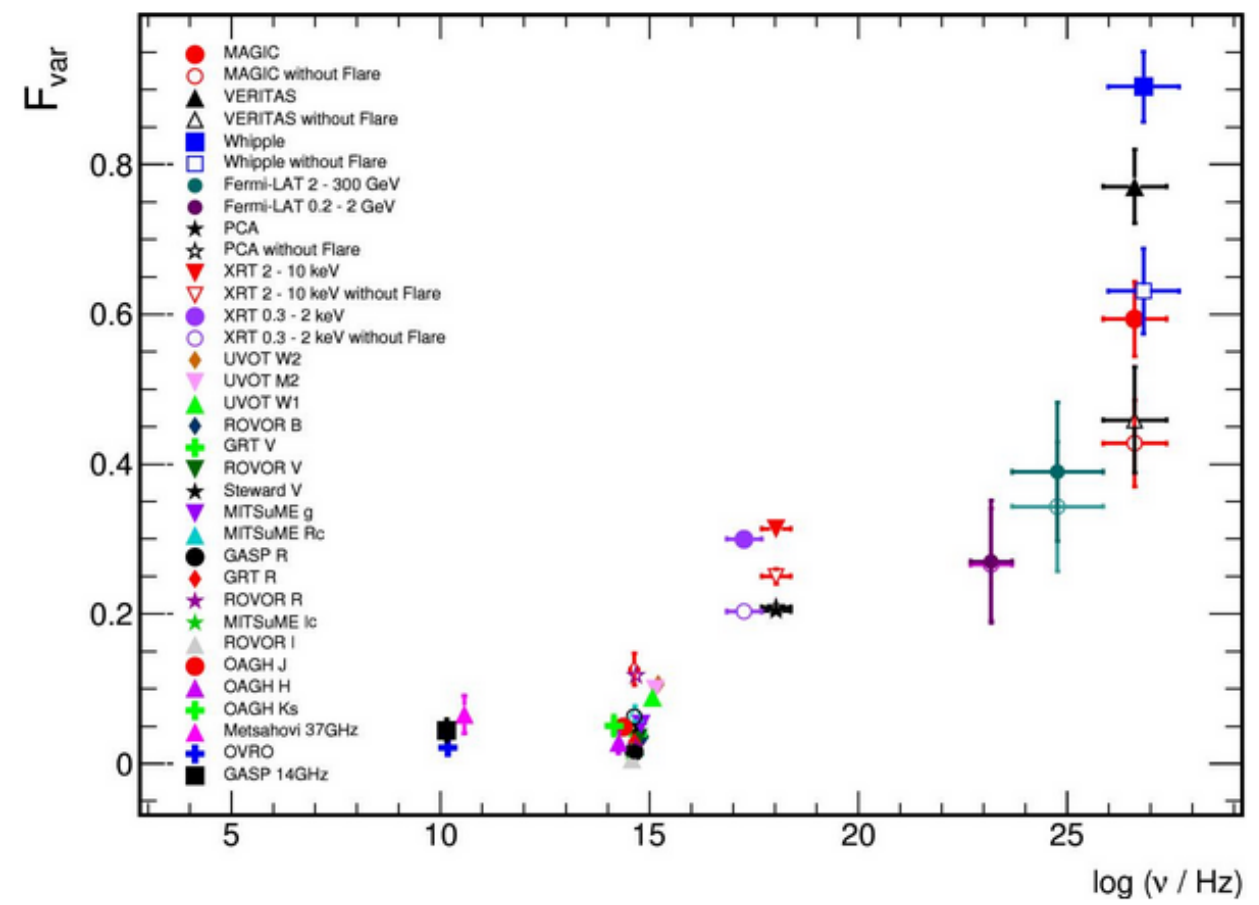

Fig. 27. Fractional variability, $F_{v a r}$ at different frequencies for Mrk 501 from 2009 observation campaign. $F_{v a r}$ shows increasing trend with frequency. Adapted from [151] with permission. 


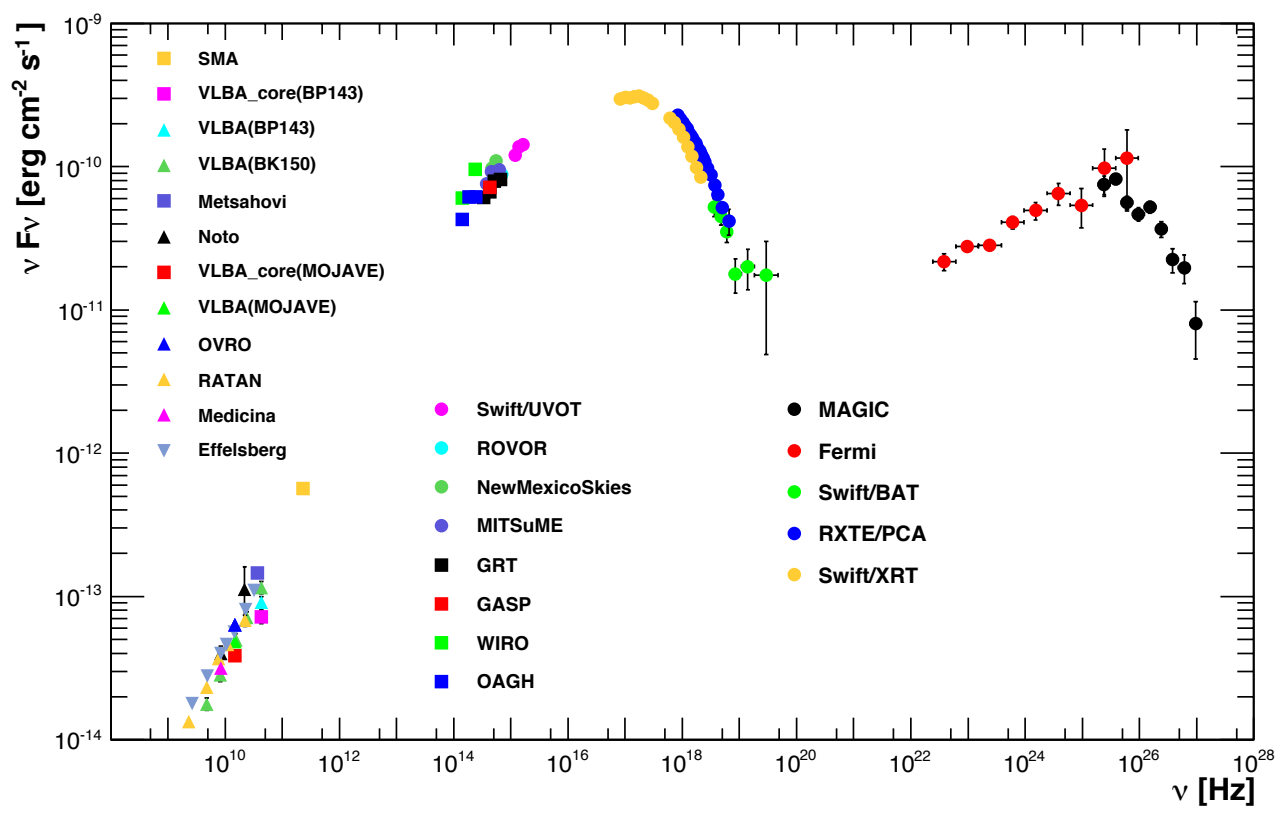

Fig. 28. SED of Mrk 421 spanning observations from radio to VHE $\gamma$-rays during observation campaign conducted during 19 January - 1 June 2009. Adapted from 147] with permission. 


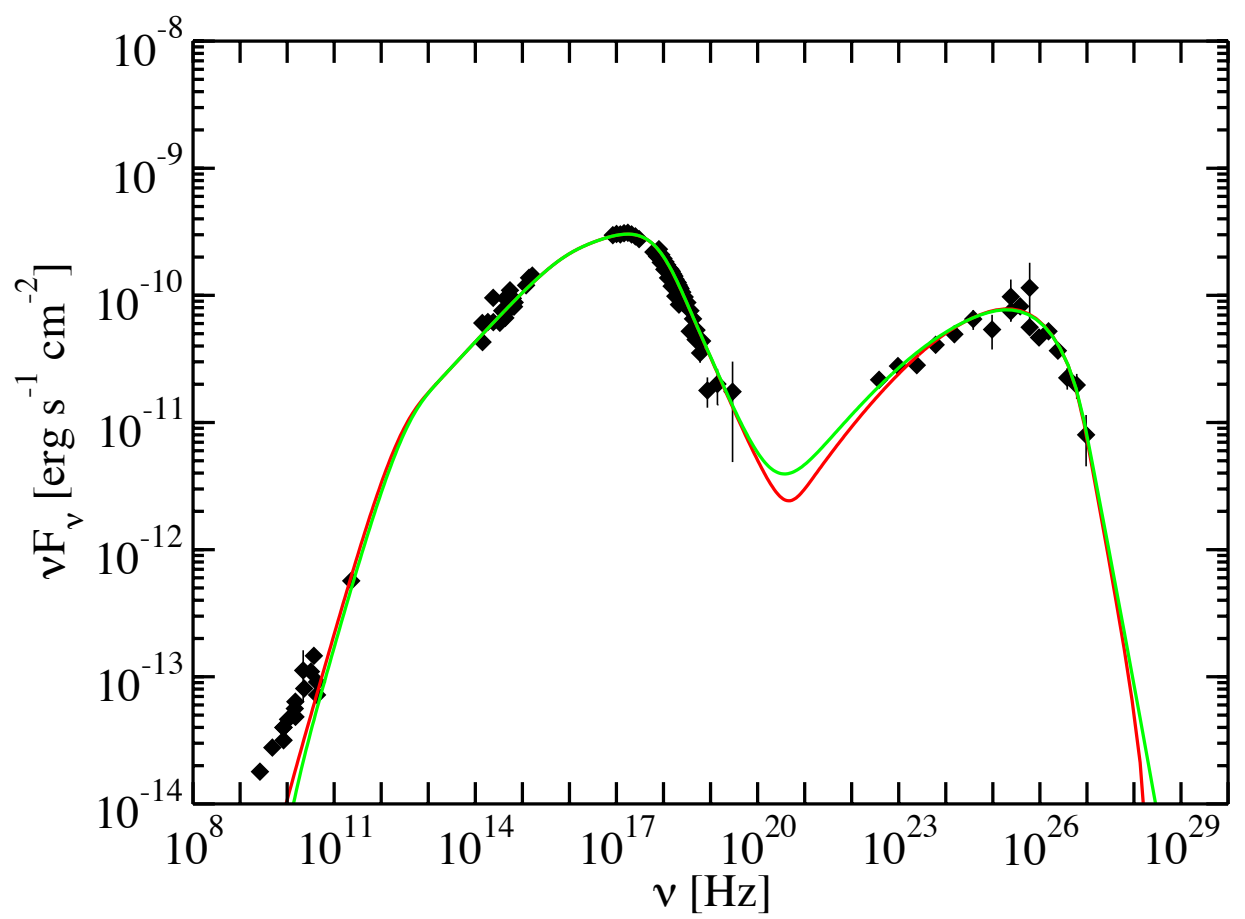

Fig. 29. SED of Mrk 421 fitted with one-zone SSC model. Red curve shows SSC for variability time scale $t_{v a r}=1$ day and green curve corresponds to $t_{v a r}=1$ hour. Adapted from [147] with permission. 


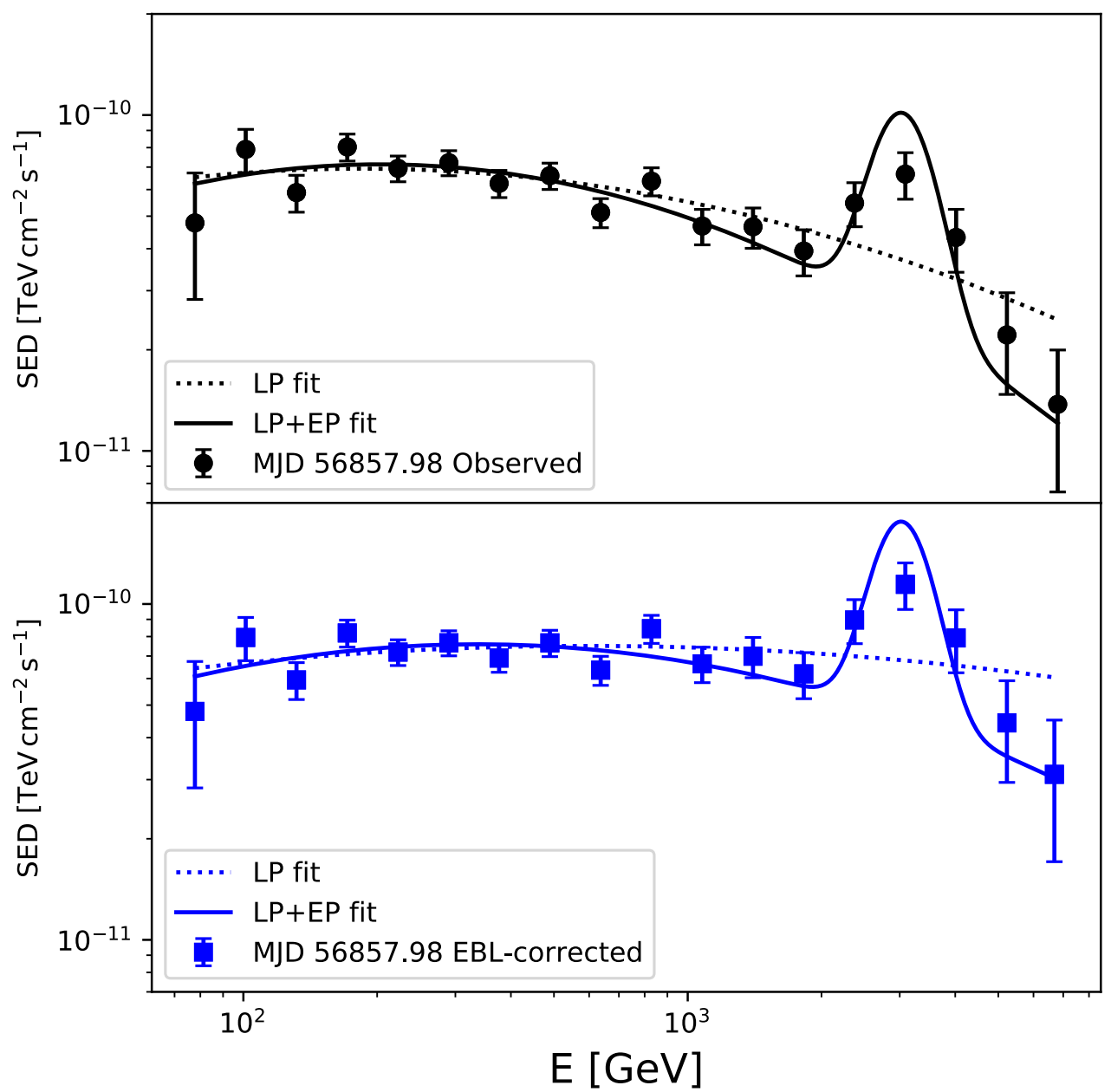

Fig. 30. VHE SED of Mrk 501 for 19 July 2014 from MAGIC telescope showing narrow feature at $\sim 3 \mathrm{TeV}$. Top and bottom panels show observed spectrum and spectrum corrected for effect of absorption by extragalactic background light. Adapted from [176] with permission. 


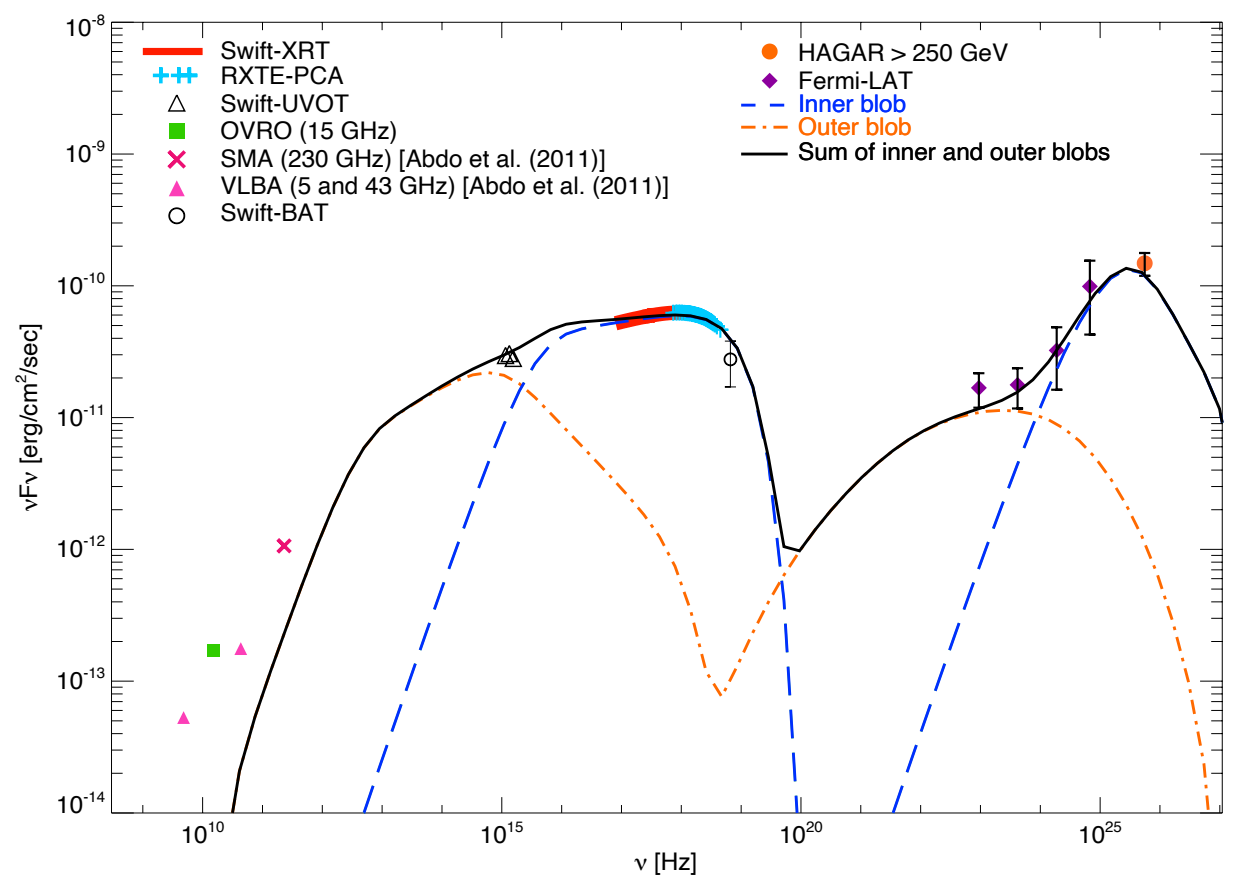

Fig. 31. Mrk 501 SED from April-May 2011 observations fitted with two-zone SSC model, with emission from inner zone, shown by blue dashed line and outer zone by orange dashdotted line. Adapted from [196] with permission. 


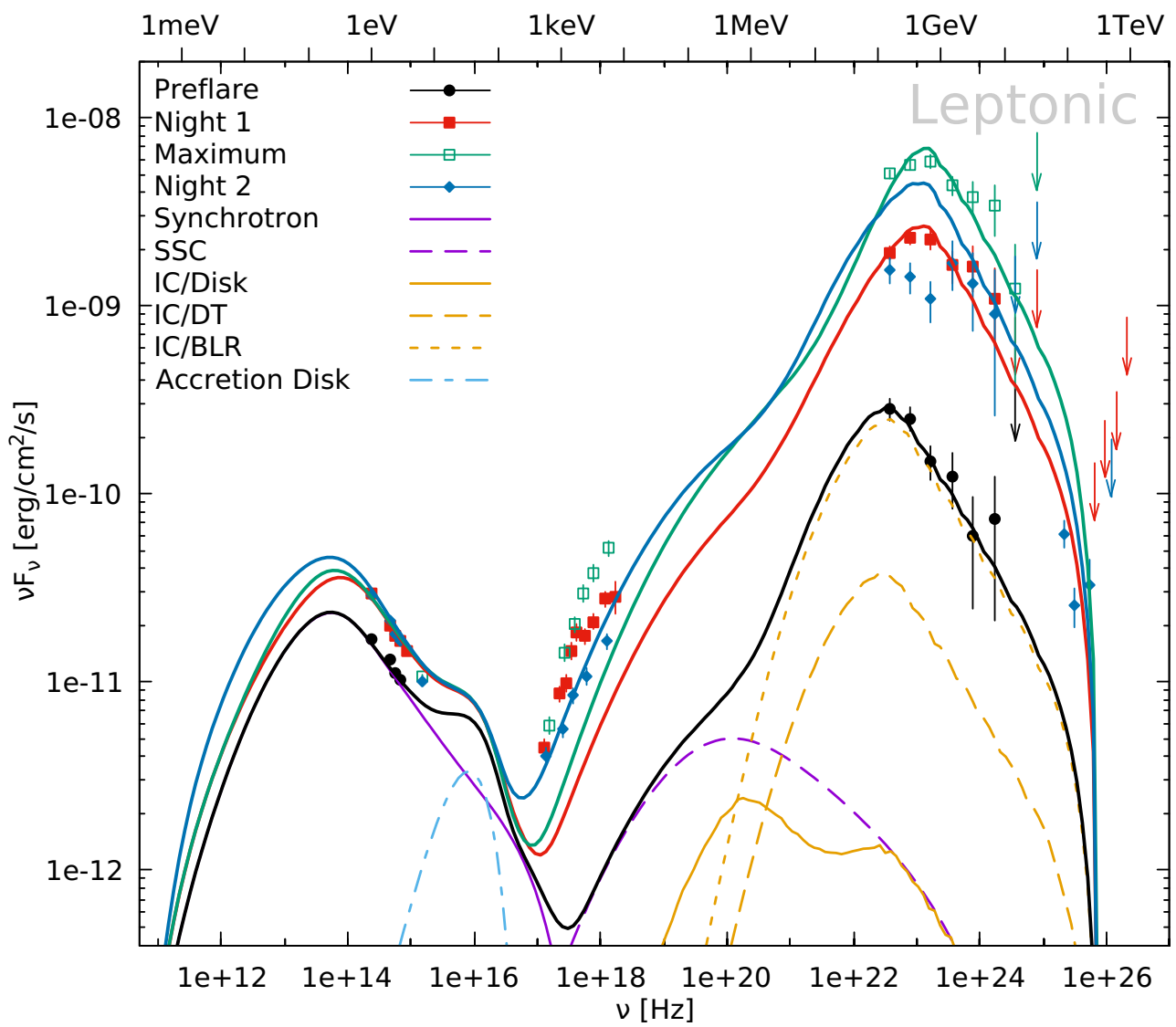

Fig. 32. SED of 3C279 during flaring episode of June 2015, modelled with a combination of SSC and EC. For pre-flares state, various model components are shown. Adapted from 199 with permission. 


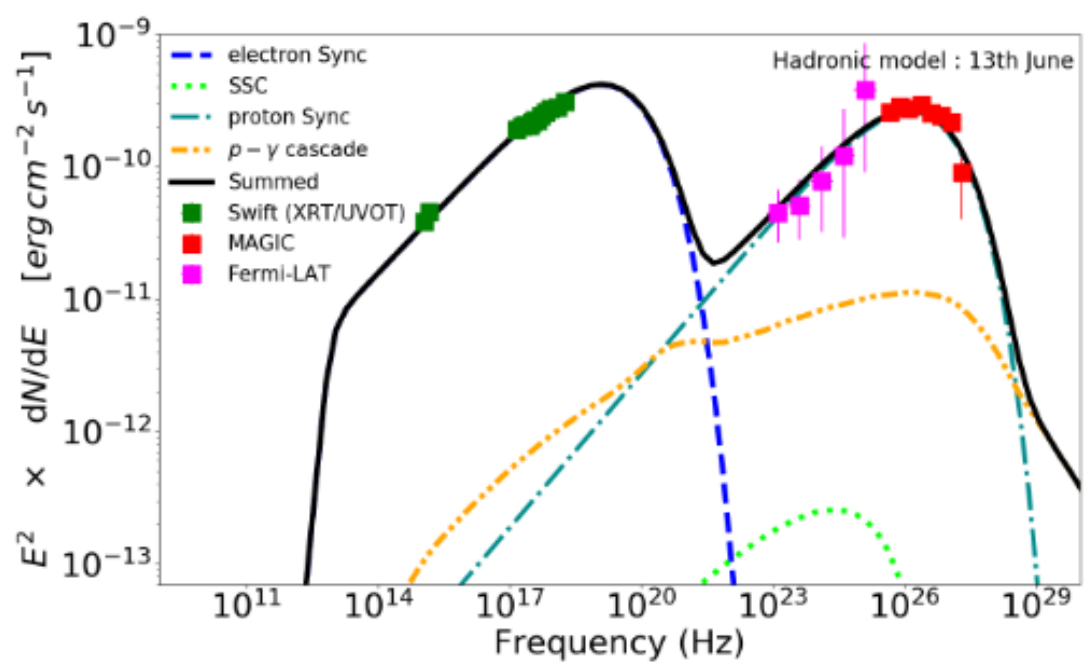

Fig. 33. 1ES1959+650 SED from flare state observations in 13 June 2016 fitted with onezone hadronic model. Data from different instruments are marked by symbols given in the legend. Various model components are shown. Higher enegy peak is dominated by synchrotron radiation from relativistic protons. Adapted from [140] with permission.

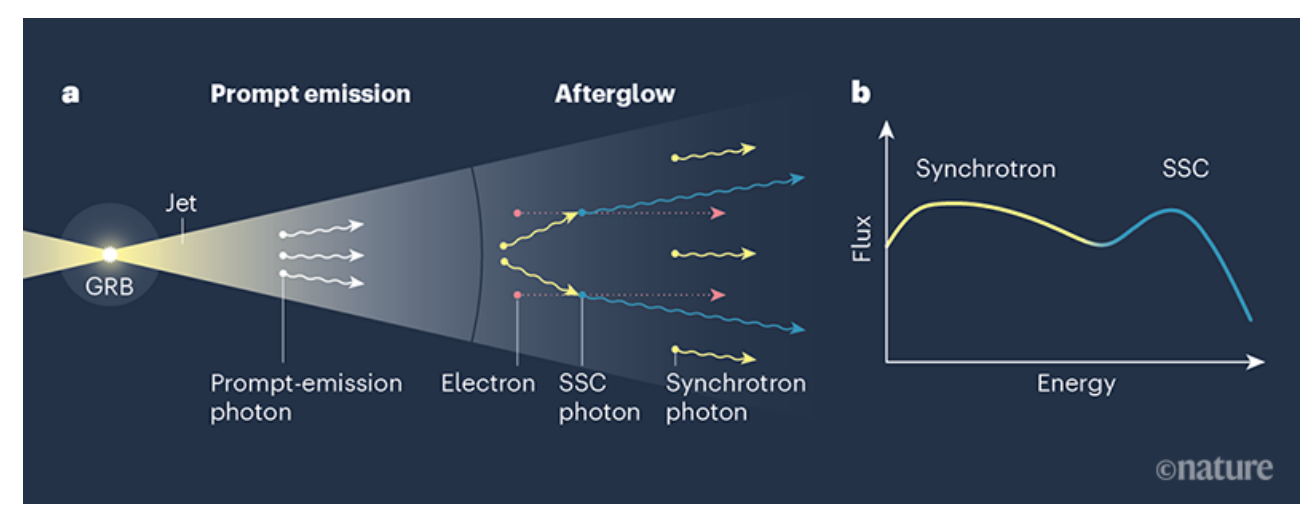

Fig. 34. Prompt and afterglow emission from GRB. This figure is taken from 222$]$. 


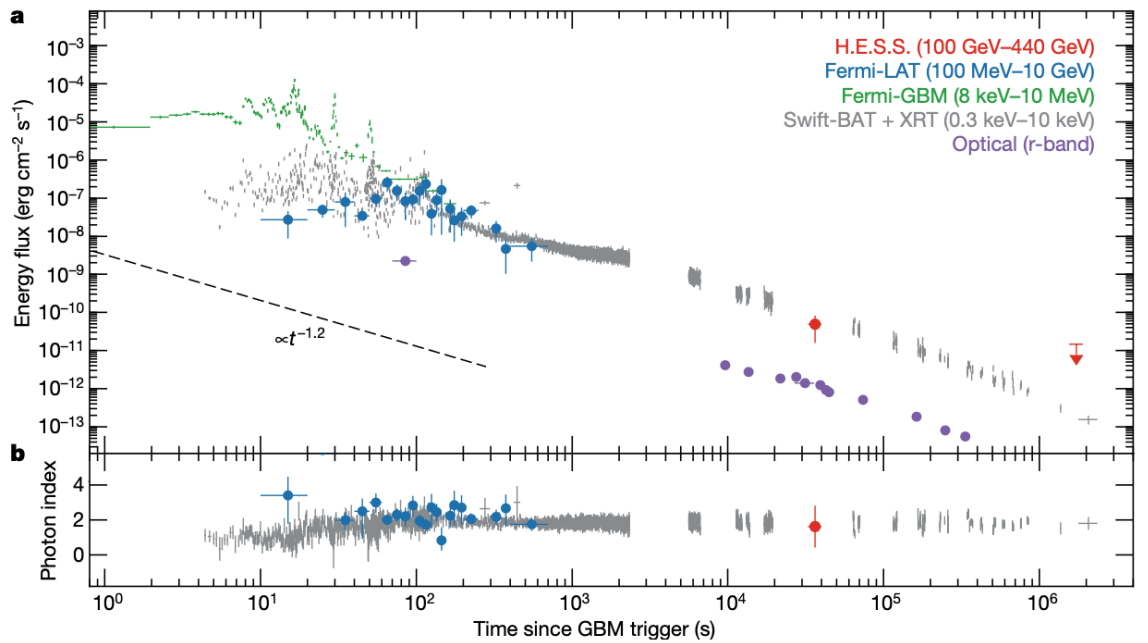

Fig. 35. Multiwavelength lightcurve of GRB 180720B in $\gamma$-rays, X-rays and optical band. This figure is taken from 223 .

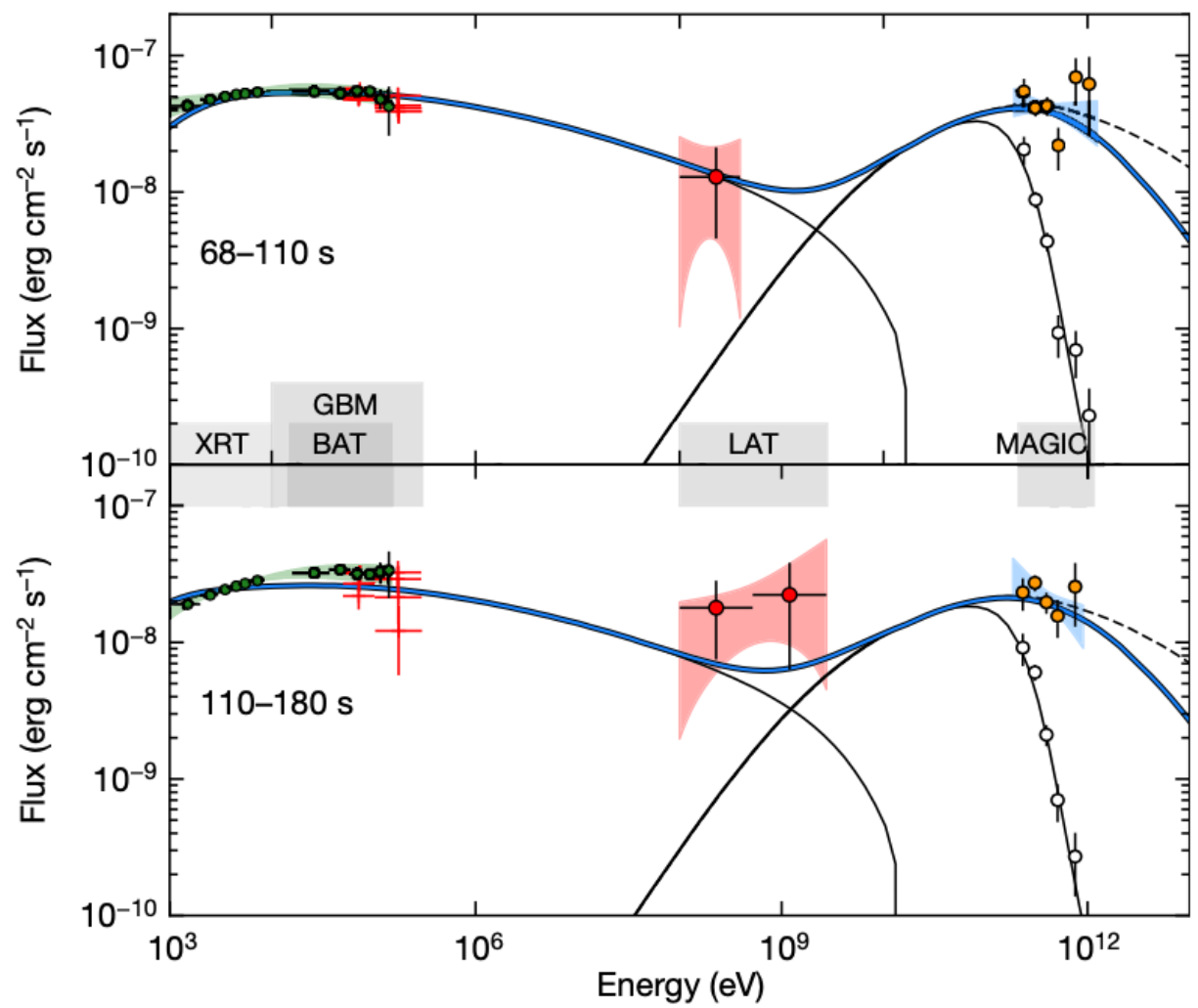

Fig. 36. Spectral Energy Distribution of GRB 190114c in the time intervals $68-110$ s and 110-180s. The broadband spectra can be best explained with synchrotron self-Compton (SSC) radiation in the external forward shock. This figure is taken from [225]. 


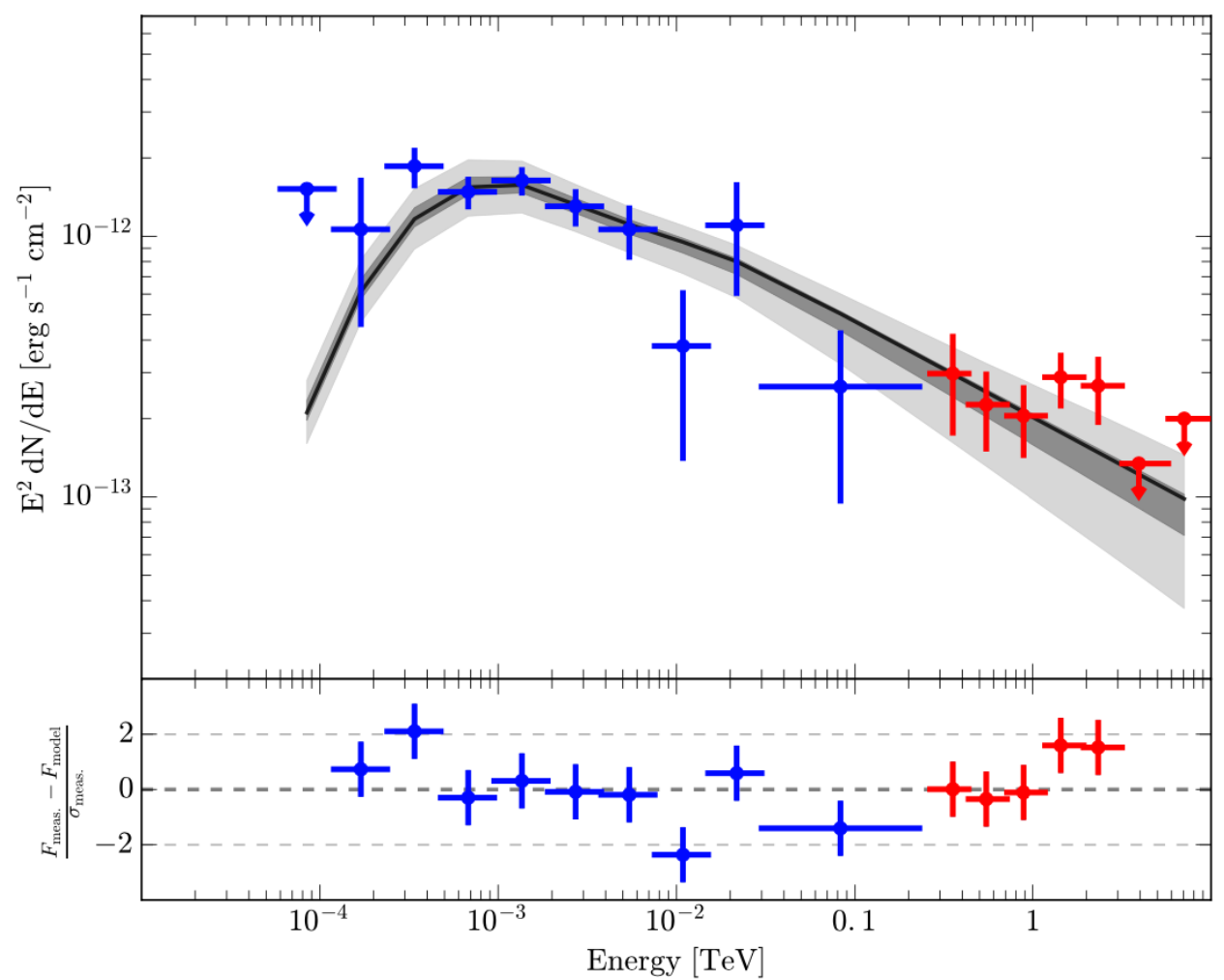

Fig. 37. The combined SED for NGC 253 obtained with data from Fermi-LAT (blue) and H.E.S.S. (red), best fitted by hadronic model. This figure is taken from [235]. 


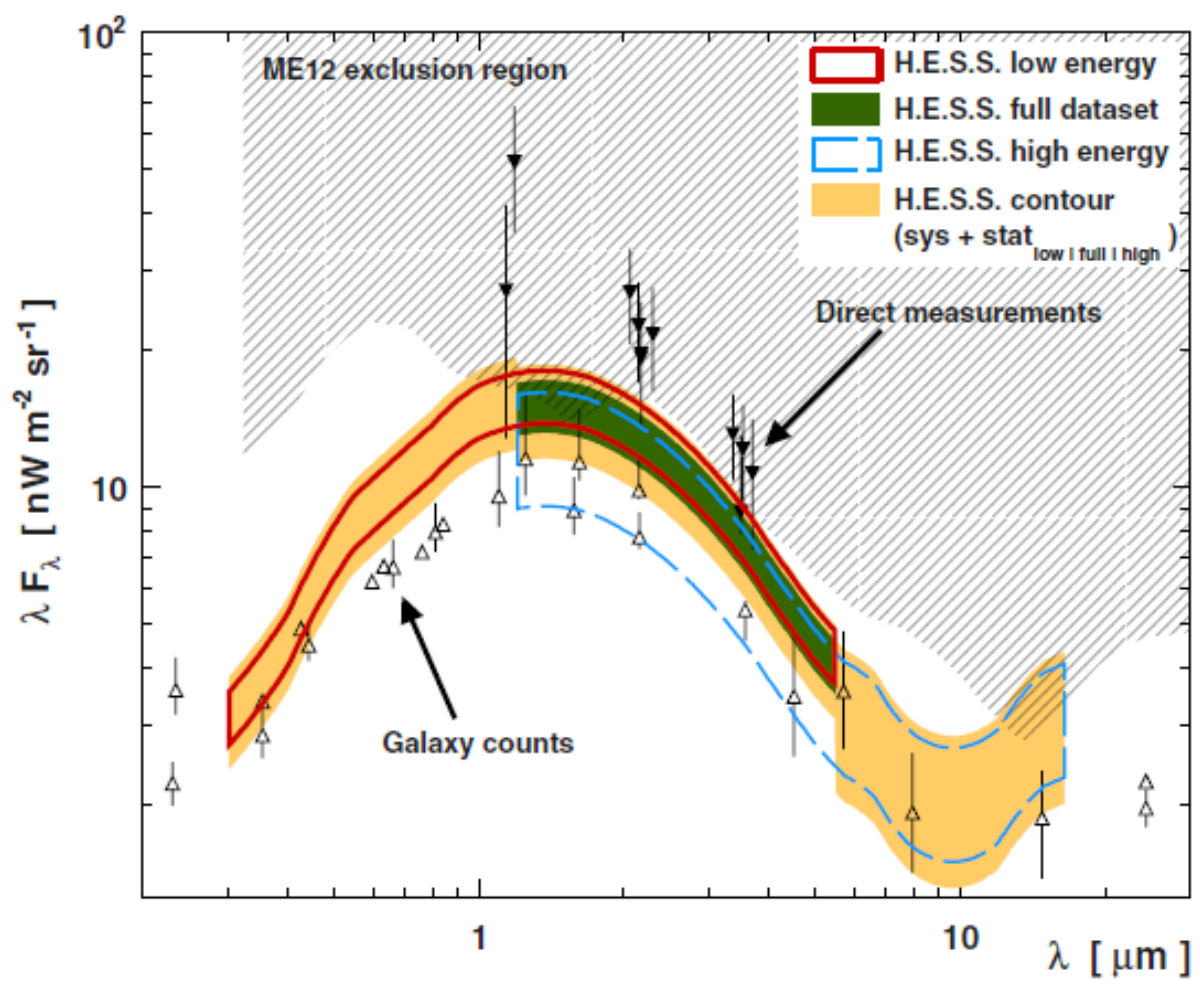

Fig. 38. EBL flux density vs wavelength for H.E.S.S. low energy, high energy measurements and full dataset consisting of seven blazars. Statistical and systematic uncertainties are taken into consideration as mentioned in the top-right legend. Lower limits based on galaxy counts and direct measurements are shown with empty upward and filled downward pointing triangles [246]. The region excluded by [253] with VHE spectra is represented by the dashed area. This figure is taken from 252 . 


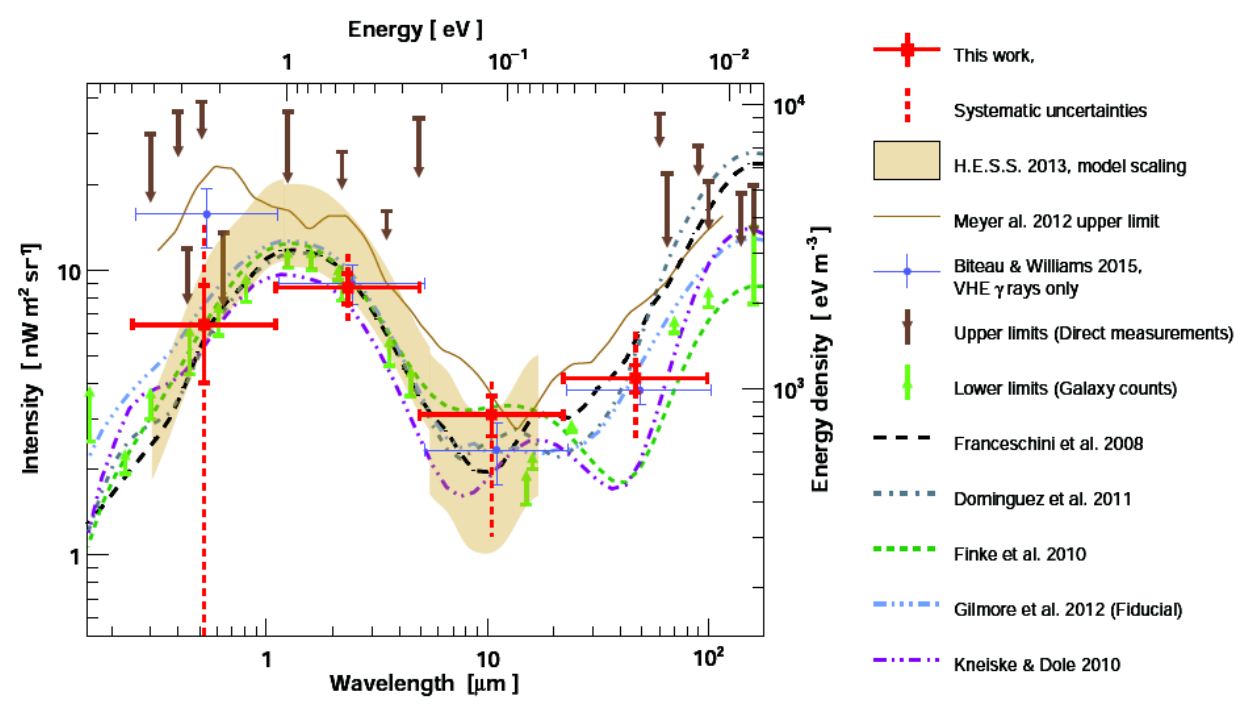

Fig. 39. Combined EBL levels (red points) compared with various constraints and models. The model-independent upper limit using VHE and HE data is shown as a thin brown line [253. The model-independent measurement of [255] restricted to the use of VHE data is represented by blue points. This figure is taken from [254]. 

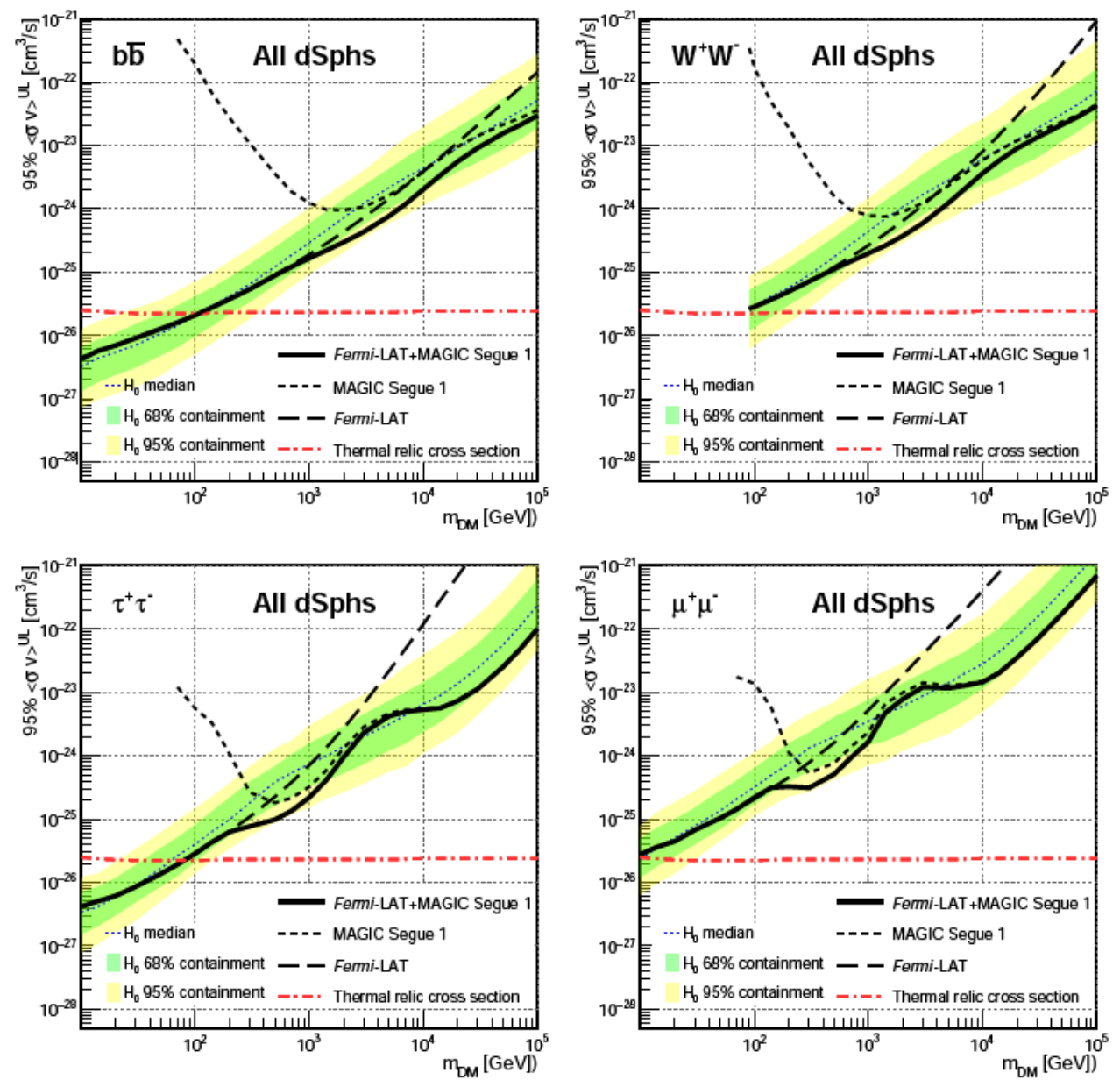

Fig. 40. 95\% confidence level upper limits on $\langle\sigma v\rangle$ for DM particles annihilating into $\bar{b} \bar{b}$ (top-left), $W^{+} W^{-}$(top-right), $\tau^{+} \tau^{-}$(bottom-left) and $\mu^{+} \mu^{-}$(bottm-right) pairs (taken from [289]). Thick solid line corresponds to limits obtained by combining MAGIC observations of Segue 1 with Fermi-LAT observations of 15 dSphs. Dashed lines correspond to individual MAGIC (short dashed) and Fermi-LAT (long dashed) limits. See [289] for further details. 